\title{
Flow Visualization Study of the MOD-2 Wind Turbine Wake
}

H. -T. Liu

Flow Industries, Inc.

June 1983

Prepared for the U.S. Department of Energy under Contract DE-AC06-76RLO 1830

Pacific Northwest Laboratory Operated for the U.S. Department of Energy by Battelle Memorial Institute 


\title{
DISCLAIMER
}

This report was prepared as an account of work sponsored by an agency of the United States Government. Neither the United States Government nor any agency thereof, nor any of their employees, makes any warranty, express or implied, or assumes any legal liability or responsibility for the accuracy, completeness, or usefulness of any information, apparatus, product, or process disclosed, or represents that its use would not infringe privately owned rights. Reference herein to any specific commercial product, process, or service by trade name, trademark, manufacturer, or otherwise, does not necessarily constitute or imply its endorsement, recommendation, or favoring by the United States Government or any agency thereof. The views and opinions of authors expressed herein do not necessarily state or reflect those of the United States Government or any agency thereof.

\author{
PACIFIC NORTHWEST LABORATORY \\ operated by \\ BATTELLE \\ for the \\ UNITED STATES DEPARTMENT OF ENERGY \\ under Contract DE-AC06-76RLO 1830
}

\begin{tabular}{|c|c|}
\hline Printed in the & 5 of America \\
\hline & \\
\hline National Tect & ation Service \\
\hline United States & f Commerce \\
\hline 5285 & oad \\
\hline Spring & 22161 \\
\hline & es A04 \\
\hline & \\
\hline & \\
\hline & Price \\
\hline Pages & Codes \\
\hline 001-025 & $\mathrm{A} 02$ \\
\hline $026-050$ & $\mathrm{~A} 03$ \\
\hline 051-075 & A04 \\
\hline $076-100$ & A05 \\
\hline $101-125$ & A06 \\
\hline $126-150$ & A07 \\
\hline $151-175$ & A08 \\
\hline $176-200$ & A09 \\
\hline $201-225$ & A010 \\
\hline $226-250$ & A011 \\
\hline $251-275$ & A012 \\
\hline $276-300$ & A013 \\
\hline
\end{tabular}


PNL-4535

UC-60

FLOW VISUALIZATION STUDY OF THE MOD-2 WIND TURBINE WAKE

\author{
H. $-\mathrm{T}$. Liu \\ J. W. Waite \\ T. R. Hiester* \\ P. H. Tacheron \\ and \\ R. A. Srnsky
}

Flow Industries, Inc.

Kent, Washington 98032

*Flowind Corporation

Kent, Washington

June 1983

Prepared for Pacific Northwest Laboratory Under Subcontract B-D5787-A-N of

Prime Contract DE-AC06-76RLO-1830

for the Department of Energy

Pacific Northwest Laboratory Richland, Washington 99352 
. 
$\underline{\text { SUMMARY }}$

Flow visualization experiments were conducted in the wake of a MOD-2 wind turbine at the Goodnoe Hills Wind Turbine Site near Goldendale, Washington. The objectives of the field experiments were to obtain qualitative and quantitative information on the characteristics of large turbine wakes under a range of atmospheric conditions. The wake characteristics under study included the wake geometry, the vertical profiles of longitudinal velocity and velocity deficit, and the rotor tip vortices. Such information is required to determine design criteria for the optimum turbine spacing and configuration of wind farms, which will be the key to the future of the wind energy program.

The experiments employed several tracer methods, including point and line sources of smoke tracers, balloons, and flow streamers, to make visible the flow phenomena of interest. The visual results were recorded on still and movie films for future analysis. Turbine 3 at the Goodnoe Hills site was used exclusively for our experiments. The field experiments, which consisted of five on-site test periods, were divided into two phases. During the first phase, preliminary tests were conducted to develop and optimize several methods for investigation of specific flow phenomena. During the second phase, the flow visualization experiments were performed using the techniques resulting from the preliminary tests.

Though several visualization methods were found to have good potential, only three were used extensively due to limitations in time (less than 4.5 months to complete the project) and budget. These methods complement each other to provide the information required for understanding the flow phenomena as specified. The most effective and versatile method used was the release of nearly vertical smoke trails from an upward-flying model rocket. The method is capable of yielding instantaneous "snapshots" of various quantities, whereas most available techniques can only provide "time-averaged" results. It is the instantaneous velocity profiles that govern the dynamic processes in the wake and the dynamic loading of downwind turbines. These processes in turn govern the evolution of the wake characteristics that are critical to the design and operation of wind farms. Ensemble averaging of our results facilitates com parison with other "time-averaged" results derived from point measurements. The second method used smoke tracers from a large smoke generator as a point 
source to track the rotor tip vortices. An innovative tether system was used to place the smoke tracers into the optimum position for visualizing the tip vortices downwind of the rotor blades. The third method used flow streamers attached to the tethered 1 ine of a parafoil kite to make visible the presence of organized vortex and random turbulent motions in the wake. This method was designed to determine visually the wake height and the persistence of the tip vortices at downwind positions.

Based on our findings, we present a typical scenario in the wake of turbine 3 at Goodnoe Hills. This scenario may be further refined when an in-depth understanding of the wake characteristics is gained. In general the wake tends to veer to the south for a westerly wind as a result of the complex topography at the site. The flow approaching the turbine is affected by the rotating blades upwind and a momentum deficit develops gradually as the flow approaches the turbine. We find that the profiles of the velocity deficit, especially the instantaneous profiles at $1 D$ downwind or closer (where $D$ is the rotor diameter), are disk-1ike. The rotation of the turbine blades generates two helical vortices that wrap around the outer edge of the disk-like near wake. The relatively low solidity of the turbine blades, as compared to a solid obstacle, effectively reduces the momentum deficit and the disturbance to the flow passing through the turbine. Consequently, very little turbulence is created as the wind passes the rotating blades. Higher levels of turbulence exist at locations further downstream. Thus, the turbulence level appears to increase initially with increasing downwind distance, at least up to 6D beyond which we did not conduct any experiments. The increase in the turbulence level is the result of flow instabilities, such as current shear or vortex instabilities, and of turbulence-vortex interactions.

One of the most important discoveries is the presence of extensive vortex stretching that creates long vortex spouts shortly after the initial generation of the tip vortices. This phenomenon has been observed in the wake of a MOD-0 turbine, but with much less intensity. Apparently, the vortex stretching 
is the result of a form of vortex instability, possibly coupled with ground effects, which has not been subject to much investigation. On one hand, the vortex stretching hastens the breakup of the tip vortices, which leads to a high turbulence level in the near wake. On the other hand, we observed several extremely long spouts that shot away from the turbine wake at a speed as high as $50 \mathrm{~m} / \mathrm{s}$. The escape of many 1 ong spouts from the wake may result in appreciable leakage or spillage of kinetic energy from the wake, which is otherwise available for turbulence generation when the vortices break up at downwind stations. The combined effect of the two competing mechanisms cannot be determined until more information is gathered. However, we observed that the tip vortices are not likely to have a significant "catastrophic" impact on turbine structures placed 4D downwind and beyond. Further investigation is required to determine the long-term impact of tip vortex effects as compared with that of wake turbulence from the structural-fatigue point of view.

Comparison of our results, derived from the instantaneous wake profiles, with a simple numerical prediction using the Lissaman wake model tends to show an overprediction in the wake growth and underprediction in the velocity deficit. Although the comparison is not a definitive verification of the Lissaman model due to our limited data set, its implications are consistent with the above scenario in that the wake turbulence is lower than expected. In essence, weak wake turbulence would limit entrainment and mixing and, therefore, wake growth. Accordingly, recovery of the momentum deficit would slow down. In practice, the turbine spacing would have to be increased to achieve the same array efficiency. Further research is recomended to verify and refine the scenario so that it can be used optimally in developing wind turbine spacing criteria and to maximize turbine array performance. 


\section{ACKNOWLEDGMENTS}

The authors would like to extend our thanks for the cooperation and assistance provided by the site personnel of the Boeing Engineering Company, Pacific Northwest Laboratory (PNL), and the Bonneville Power Administration (BPA). We would also like to acknowledge the assistance of the members of the Wind Turbine Site (WTS) Test Project Review Board, which included representatives from the above organizations and the National Aeronautics and Space Administration (NASA). The enthusiastic support from Mr. Don Hadley, Project Monitor at PNL, deserves special acknowledgment. In addition to being a liaison between $\mathrm{F}$ low and other organizations, he actively participated in all phases of the field experiments. The authors, thereby, consider him as a member of our project team. 


\section{TABLE OF CONTENTS}

SUMMARY

iii

LIST OF FIGURES . . . . . . . . . . . . . . . . . . . ix

LIST OF TABLES ........................ . .

NOMENCLATURE . . . . . . . . . . . . . . . . . . . . . xi

1. INTRODUCTION . . . . . . . . . . . . . . . . . . . 1

2. CONCLUSIONS AND RECOMMENDATIONS . . . . . . . . . . . . . 3

2.1 CONCLUSIONS . . . . . . . . . . . . . . . . . 3

2.2 RECOMMENDATIONS . . . . . . . . . . . . . . 7

3. BACKGROUND OF THE WAKE PROBLEM . . . . . . . . . . . . . 11

3.1 MODELS OF WIND TURBINE WAKES . . . . . . . . . . . . . 11

3.1 .1 Velocity Deficit . . . . . . . . . . . . 12

3.1 .2 Wake Geometry ................. 15

3.1.3 Turbulence Characteristics . . . . . . . . 16

3.1 .4 Rotor Tip Vortices . . . . . . . . . . . 17

3.1.5 Practical Models . . . . . . . . . . . . 18

3.2 CRITICAL ASSUMPtions TO BE TESTED . . . . . . . . . 22

3.2.1 Velocity Deficit Profile . . . . . . . . . 22

3.2 .2 Wake Geometry . . . . . . . . . . . . 23

3.2.3 Wake Turbulence Characteristics . . . . . . . . 23

3.2.4 Role of Rotor Tip Vortices . . . . . . . . . . 23

4. EXPERIMENTAL STRATEGY AND EXECUTION . . . . . . . . . . . . 25

4.1 THE GOODNOE HILLS SITE . . . . . . . . . . . . . 25

4.2 EXPERIMENTAL STRATEGY . . . . . . . . . . . . . . 27

4.3 EXECUTION OF EXPERIMENTS . . . . . . . . . . . 29

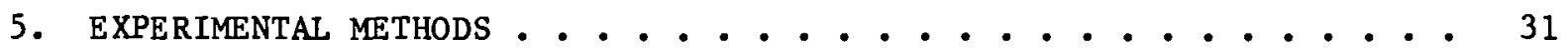

5.1 METHODS OF FLOW VISUALIZATION . . . . . . . . . . 31

5.1 .1 Vertical Profiles of Longitudinal Velocity . . . . . 31

5.1 .2 Rotor Tip Vortices . . . . . . . . . . . 38

5.1.3 Wake Height ............... 43

5.2 PHOTOGRAPHIC EQUIPMENT . . . . . . . . . . . . . . 44

5.3 Data Analysis . . . . . . . . . . . . . . . 44

5.3.1 Rocket Trail Digitization . . . . . . . . . 45 


\section{TABLE OF CONTENTS (cont.)}

5.3.2 Rocket Trail Data Analysis . . . . . . . . . . 46

5.3.3 Tracking the Wake Centerline ........... 51

5.3.4 Computer Program ............... 53

5.3.5 Estimated Errors in the Rocket Trail Data . . . . . 54

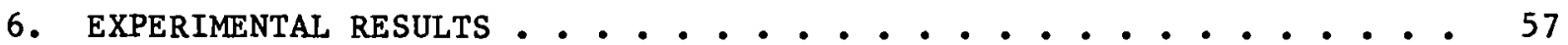

6.1 VISUAL RESULTS FROM ROCKET TRAILS . . . . . . . . . 57

6.2 ROCKET SMOKE TRAIL DATA . . . . . . . . . . . . . 64

6.3 WAKE HEIGHT DETERMINATIONS . . . . . . . . . . . 68

6.4 VELOCITY DEFICIT . . . . . . . . . . . . . 82

6.5 COMPARISON WITH A SIMPLE NUMERICAL MODEL . . . . . . . . 84

6.6 ROTOR TIP VORTICES . . . . . . . . . . . . . 90

6.6.1 Visual Results from Point Release of Smoke Tracers . . . 92

6.6.2 Visual Results from Rocket Smoke Trails ........ 103

6.6.3 Visual Results from Flow Streamers . . . . . . . 106

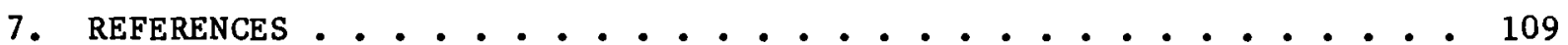

APPENDIX A - ROCKET DESIGN AND ASSEMBLY . . . . . . . . . . . A-1

APPENDIX B - IMPLEMENTATION OF THE TIP VORTEX VISUALIZATION . . . . . B-1

APPENDIX C - COMPUTER ANALYSIS . . . . . . . . . . . . . . C-1 
4.1 Goodnoe Hills Test Site . . . . . . . . . . . . 26

5.1 Conceptual sketch of Rocket Trail Experiments . . . . . . . 32

5.2 Sketch of Rocket Assembly ................. 33

5.3 Photographs of Rocket Assembly . . . . . . . . . . . 34

5.4 Geometrical Layout of Rocket Experiments . . . . . . . . . 36

5.5 Placement of Stations Downwind of Turbine $33 \ldots$. . . . . . 37

5.6 Setup of Tethered Cable System for Positioning Smoke Pot . . . . 40

5.7 Parafoil Kite with Flow Streamers . . . . . . . . . . 42

5.8 Horizontal Correction of Rocket Smoke Trail Position Data . . . 47

5.9 Vertical Correction of Rocket Smoke Trail Position Data . . . . 48

5.10 Vertical Segment Correction Between Successive Rocket Smoke Trail Position Data . . . . . . . . . . 50

5.11 Geometry of the Curved Wake of Turbine $\equiv_{3}$. . . . . . . . . 52

6.1 Sequential Pictures of a Single Smoke Trail at $1 / 2 \mathrm{D}$ and

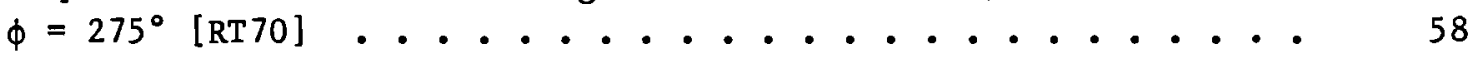

6.2 Sequential Pictures of Two Smoke Trails [RT30 and RT31] . . . 61

6.3 Enlarged Photographs of Smoke Trails Selected from Figure 6.2

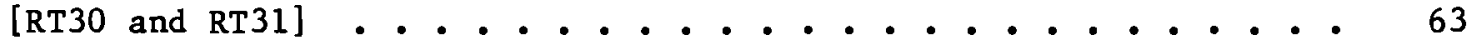

6.4 Sequential Pictures of a Single Smoke Trail at $6 \mathrm{D}$ and

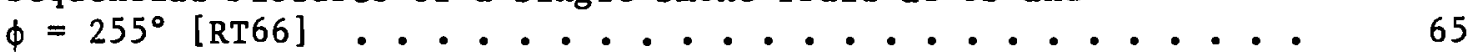

6.5 Velocity Profile at an Effective Off-Axis Position of -6 Meters (Background) .......................

6.6 Rocket Trail Position Data Corrected for Lens Distortion (Background) ..................... 71

6.7 Rocket Trail Position Data Corrected for Lens Distortion [RT30] . 73

6.8 Velocity Profile at an Effective Off-Axis Position of +12 Meters [RT30] . . . . . . . . . . . . 74

6.9 Rocket Trail Position Data Corrected for Lens Distortion [RT66] $\quad 75$

6. 10 Velocity Profile at an Effective Off-Axis Position of -48 Meters [RT66] . . . . . . . . . . . . . . . . 76

6.11 Rocket Trail Position Data Corrected for Lens Distortion [RT42] $\quad 77$

6.12 Velocity Profile at an Effective Off-Axis Position of +3 Meters [RT42] ................ 78

6.13 Velocity Profile at an Effective off-Axis Position [RT70] of -8 Meters . . . . . . . . . . . . . . . . 


\section{LIST OF FIGURES (cont.)}

6.14 Down-Axis View of MOD-2 Wake Growth . . . . . . . . . . 81

6.15 Mean Downwind Velocity Deficit Profiles at the 1D, 4D, and 6D Positions . . . . . . . . . . . . . . . 85

6.16 Comparison of Lissaman Model and Measurements of Wake Height . . . 87

6.17 Comparison of Lissaman Model and Measurements of Velocity Deficit 89

6.18 Conceptual Drawing of the Rotor Tip Vortices with Relation to the Smoke Observation . . . . . . . . . . . . . . . . 93

6.19 Side View of Rotor Tip Vortices [SP24] . . . . . . . . . . 94

6.20 Side View of Rotor Tip Vortices that Persist as far as $1.5 \mathrm{D}$ [SP17] 95

6.21 Downwind Views of the Tip Vortices [SP24] . . . . . . . . 98

6.22 Closeup of Forward Spouts [SP24] ................ 99

6.23 Closeup of Reverse Spouts [SP22] . . . . . . . . . 100

6.24 Two Spouts in the Transition State from the Forward to the Reverse Configuration . . . . . . . ....... 101

6.25 Sequential Pictures of a Single Smoke Trail at $1 \mathrm{D}$ and

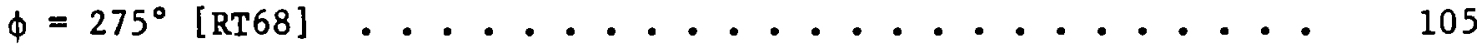

6.26 Four Series of Pictures of the Motion of Streamers Caught in the Tip Vortices [FS73] ............... 107

\section{LIST OF TABLES}

6.1 Summary of Rocket Smoke Trail Run Conditions and observations . . 59

6.2 Sumary of Experiments Using Large Smoke Generators and Flow Streamers ............................ 
NOMENCLATURE

\begin{tabular}{|c|c|}
\hline Symbol & Meaning \\
\hline$c_{t}$ & turbine thrust coefficient \\
\hline D & MOD-2 rotor diameter $(94 \mathrm{~m})$ \\
\hline$\left(d_{\mathrm{w}} / \mathrm{dx}\right)_{a}$ & $\begin{array}{l}\text { growth rate due to ambient turbulence } \\
\text { actual vertical smoke trail elevation }\end{array}$ \\
\hline $\mathrm{L}$ & actual horizontal separation between smoke trails \\
\hline $\mathrm{m}$ & $\begin{array}{l}\text { measured ground level arc length between successive rocket trails } \\
\text { parameter }\left(=U_{0} / u(x=0)\right)\end{array}$ \\
\hline P & inferred arc length between successive rocket trails \\
\hline $\mathbf{R}$ & rocket launch pad/camera distance \\
\hline$R_{w}$ & wake radius at a local downstream distance \\
\hline 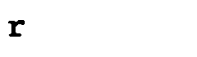 & radial distance from wake centerline \\
\hline$S_{x}$ & measured arc length along a horizontal circle of constant scale \\
\hline$S_{z}$ & $\begin{array}{l}\text { measured arc length to a particular smoke trail elevation } \\
\text { time }\end{array}$ \\
\hline $\mathbf{u}$ & local velocity \\
\hline$u(x=0)$ & velocity at the rotor disk \\
\hline $\mathrm{U}_{0}$ & longitudinal free-stream wind speed \\
\hline V & transverse free-stream wind speed \\
\hline$x, y, z$ & downwind, transverse, and vertical distances \\
\hline 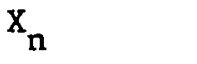 & near wake length \\
\hline$\alpha$ & turbulence intensity \\
\hline 及 & camera viewing angle from launch pad to smoke trail \\
\hline$y$ & wind direction at rocket 1 aunch pad \\
\hline$\Delta \mathrm{T}$ & measured lapse rate at PNL tower over $96.6 \mathrm{~m}$ \\
\hline$\Delta \mathrm{l}$ & local centerline velocity deficit \\
\hline$\Delta \mathrm{U}_{\mathrm{m}} / \mathrm{U}_{\mathrm{O}}$ & mean internal velocity deficit \\
\hline$\delta$ & camera viewing angle from launch pad to height h \\
\hline$f$ & defined as $\bar{\gamma}-\theta_{0}$ \\
\hline & turbine axis angle \\
\hline $\mathrm{PA}$ & wind direction at BPA tower \\
\hline & effective turbine axis angle \\
\hline
\end{tabular}




\section{NOMENCLATURE (cont.)}

$\begin{array}{ll}\theta_{\mathrm{m}} \text { Symbol } & \text { Momentum thickness } \\ \theta_{0} & \text { wind direction at turbine }{ } 3 \\ \theta_{\mathrm{PNL}} & \text { wind direction at PNL tower } \\ \sigma_{\mathrm{v}} & \text { standard deviation of the transverse wind speed } \\ \sigma_{\theta} & \text { standard deviation of the wind direction } \\ \phi & \text { baseline orientation of rocket launch pad } \\ \chi & \text { actual horizontal smoke trail advection distance }\end{array}$




\section{INTRODUCTION}

The Research and Technology Division of Flow Industries, Inc., (referred to as Flow hereafter) and Flow's subcontractor, the Flowind Corporation, performed a series of flow visualization experiments in the wake of a MOD-2 wind turbine at Goodnoe Hills, near Goldendale, Washington. The full-scale experiments, which were completed in five on-site test periods, used several visualization methods (including smoke tracers, smoke trails from model rockets, neutrally buoyant balloons with or without smoke release, and flow streamers attached to a kite string) to observe qualitative characteristics of the wake of a single turbine. However, 1 imited quantitative data about the wake were obtained from the results of the flow visualizations, which were recorded on both still and movie film. This is the project's final report, covering the work completed during the period from June to September 1982.

It is necessary to understand the wake characteristics of a single large wind turbine to optimize the design and operation of future arrays of large wind turbines in terms of turbine separation and orientation. The most important wake characteristics include the wake geometry (i.e., the maximum wake height and width and the cross-sectional area of the wake), the turbulent velocity fluctuations, the mean velocity deficit, and the rotor tip vortices. Knowledge of wake geometry is crucial for verifying the wind turbine array models that are based on conservation of the momentum deficit, since those models directly relate the wake centerline velocity deficit to the downstream growth of the wake. Most of the previous research emphasis on the problem of wind turbine spacing has been related to the momentum deficit and consequent loss of energy capture. However, it has been suggested that high levels of turbulence and the persistence of rotor tip vortices in wind turbine wakes, and the resulting impacts on the design and operation of wind turbines, might turn out to be the most crucial aspects of wind array design.

The features of large-scale wind turbine wakes remain poorly documented and possibly misunderstood. The flow visualization experiments of this study are a rational and needed precursor to detailed quantitative studies of wind turbine wakes. The overall objective of this project was to document qualitative characteristics of some of the most basic wake features, such as wake 
geometry, velocity deficit profile shape, and rotor tip vortices. The flow visualization experiments proved to be capable of providing a crucial understanding of large-scale wake behavior that cannot be obtained in sma11-scale tests.

The specific objectives of this study were as follows:

1. To determine the geometry of the MOD-2 wind turbine wake, in terms of wake height and width as a function of downstream distance (up to 10 rotor diameters downstream), under two conditions of atmospheric stability.

2. To estimate the mean velocity deficit at several downstream stations in the turbine wake.

3. To investigate the behavior of the rotor-generated vortices, particularly their configuration and persistence.

Section 2 of this report summarizes the principal conclusions of the study and presents recommendations for future work. In Section 3, we discuss the wind turbine wake problem and provide background information that helped formulate our experimental strategies, which are presented in Section 4 . Section 5 details the experimental techniques and data analysis methods. Section 6 concludes the main text of this report with a presentation and discussion of the data. We include further information on the experimental methods in Appendices $A$ and $B$, and provide details on the computer analysis of the data, including the computer program, in Appendix $C$. In addition, a 16-mm movie film, entitled "Flow Visualization in the Wake of a MOD-2 Wind Turbine" (Liu et al. 1982), has been produced to summarize the visual results. This film, referred to frequently in this report, is an important part of the final results of this study. 


\section{CONCLUSIONS AND RECOMMENDATIONS}

In this section, the conclusions from our flow visualization study are presented and recommendations for future work are outlined.

\subsection{CONCLUSIONS}

Several visualization methods were successfully developed to obtain qualitative and quantitative information on the wake height, the vertical profiles of longitudinal velocity and velocity deficit, and the rotor tip vortices downwind of a MOD-2 wind turbine. The first method was the release of nearly vertical smoke trails from model rockets. It was the most effective and versatile method used in that it is capable of obtaining information for all the flow phenomena investigated in this study. From time-lapse photographs of the smoke trails (for 3 to 15 seconds, depending on the intensity of the wake turbulence), we derive instantaneous "snapshots" of the velocity profiles and velocity deficit that can be ensemble-averaged to compare with other timeaveraged results. The importance of the instantaneous nature of the wake cannot be overemphasized. The instantaneous velocity profiles govern the dynamic processes in the wake and the dynamic loading of downwind turbines. These processes include current shear and vortex instability, turbulence-vortex interaction, and turbulence entrainment, which in turn govern the evolution of the wake characteristics that are critical to the design and operation of wind farms. The second method was the point release of smoke tracers from a cable tethered to the catwalk of the turbine tower. This method was designed to provide visual observations of the rotor tip vortices in terms of their generation and persistence. The third method used flow streamers attached to the tethered line of a parafoil kite. The streamers were deployed to make visible the presence of organized vortex and random turbulent motions in such a way that we could estimate the wake height and the persistence of tip vortices at downwind stations.

The visualization results, in the form of still pictures and movie film, allowed us to evaluate the wake of turbine 3 . During periods of westerly winds, the wake path downwind of turbine 非 3 systematically veers to the south with increasing downwind distance. This phenomenon, evident from the movie 
records (Liu et al. 1982) and validated by the discrepancy between the measured wind directions at the BPA and PNL towers, is a result of the complex terrain features at the Goodnoe Hills site. The curved wake trajectory created some difficulty in accurately placing the wake centerline with respect to the point of rocket release; though an interpolative scheme was derived to overcome the problem.

The profiles of the velocity deficit at $1 \mathrm{D}$ and closer, where $\mathrm{D}$ is the rotor diameter, were disk-like. Apparently, the approaching flow is affected by the blockage of the rotating turbine blades upwind and a momentum deficit is developed gradually as the flow approaches the turbine. The process of building up the momentum deficit has a large time scale that smooths out the periodicity of the blade rotation. The relatively low solidity of the rotating blades, as compared to a solid obstacle, reduces the magnitude of the momentum deficit and the disturbance to the flow passing through the turbine. The centerline velocity deficit is estimated to be about $50 \%$ at $1 \mathrm{D}$ and $25 \%$ at $4 \mathrm{D}$. From the persistence of the smoke trails at $1 / 2 \mathrm{D}$ inside the wake, it appears that little turbulence is created when the wind passes through the rotating blades. The turbulence level in the wake appears to increase initially with the increasing downwind distance, at least up to $6 \mathrm{D}$, beyond which we did not conduct any experiments. The increase of the turbulence level is brought about by shear and vortex instability, turbulence-vortex interaction, and turbulent entrainment or mixing.

The wake height is determined from the smoke trails by using two criteria. For the first criterion, we take advantage of the fact that the turbulence level is higher inside than outside the wake. We define the wake height to be the vertical distance (from the ground) above which the smoke trails persist for a long time. For the second criterion, we define the wake height as the vertical distance at which the velocity deficit vanishes rapidly. For the very near wake, $x \leq 1 D$, we estimated the wake height from both criteria, and the two estimates differ only slightly. For the far wake, $x \geq 4 \mathrm{D}$, the wake height can only be estimated using the first criterion because the velocity deficit is too small to distinguish the wake edge. The inferred centerline wake height increases from $107 \mathrm{~m}$ at $1 / 2 \mathrm{D}$ to $170 \mathrm{~m}$ at $6 \mathrm{D}$, as derived from the smoke trail data. 
The rotation of the blades generates two helical vortices that wrap around the outer edge of the near wake. Though they are,coherent and organized upon generation, it is well known that the rotor tip vortices are inherently unstable, especially under the influence of ambient turbulence. Our visual results generally support this statement. One of the most important discoveries is the presence of extensive vortex stretching of the tip vortices. We observed many vortex spouts 100 m or longer that shot away from the turbine wake at speeds as high as $50 \mathrm{~m} / \mathrm{s}$. On the left side of the turbine, where the blades rotate toward the ground, "forward" spouts moving in the same direction as the blades are first observed. Abrupt reversals of a few of the forward spouts take place shortly after the spouts are generated. This vortex reversal leads to extensive stretching of the "reverse" spouts and triggers the highspeed shooting phenomenon. On the opposite side of the turbine, where the blades rotate away from the ground, no flow reversal was observed (from a single run only). The long spouts simply result from the stretching of the forward spouts. A similar phenomenon was observed in the wake of a MOD-0 turbine wake, but it was not as dramatic as that seen here. The dramatic vortex stretching is believed to be the product of a form of instability, possibly coupled with ground effects in a turbulent environment. This vortex stretching phenomenon has not been subject to much investigation. On one hand, the vortex stretching hastens the breakup of the tip vortices and therefore leads to a high turbulence level in the near wake. On the other hand, the escape of many long spouts away from the wake may result in appreciable leakage or spillage of kinetic energy out of the wake, which is otherwise available for turbulence generation when the vortices break up at downind stations. The combined effect of the two competing mechanisms cannot be clarified until more information is available. The hypothesis of energy leakage from the long spouts is, however, consistent with our qualitative observation of lower-than-expected wake turbulence. The frequency of occurrence of the long spouts should be determined to assess whether energy leakage is indeed appreciable.

From the motion of the flow streamers, it was apparent that the tip vortices are not likely to have a significant "catastrophic" impact on the turbine structure placed at 4D downwind and beyond. Further investigation is required 
to determine their long-term impact as compared with that of wake turbulence from the structura1-fatigue point of view.

The ensemble of rocket runs that was used to estimate maximum wake heights and velocity deficits at 1D, 4D, and 6D downwind of turbine 非 was compared against a group of predictions of wake heights and wake centerline velocity deficits generated by the Lissaman wake model. The purpose of the comparison was to check the internal consistency of the two quantities from the rocket data, and to indicate issues that might need further consideration for the formulation of simple numerical models. The comparison is not a definitive verification of the Lissaman model due to the limited sample of data. The comparison revealed that the wake heights and velocity deficits measured by the rocket technique were internally consistent when viewed in light of the assumption of conservation of the momentum deficit. The Lissaman model overpredicted the wake heights and underpredicted the velocity deficits.

The results of comparison are consistent with our qualitative observation of lower-than-expected wake turbulence. It is also suggested that topographically forced accelerations along the wake axis at the Goodnoe Hills site may cause a constriction of streamlines that contributes to the lower-thanpredicted wake heights. Another possibility is that the role of turbulence in the growth of wind turbine wakes for very large scale wakes, such as the MOD-2 wake, is not properly parameterized by the model. This could be true of either ambient turbulence or rotor-generated turbulence (for example, leakage of turbulence due to extreme vortex stretching). Since the model was tuned with wind tunnel data (and limited field data from smaller turbines), this could indicate that the wind tunnel models do not properly simulate large wind turbine wakes, that is, too high of a turbulence level is generated in the simulated wake. This problem requires further study, since the trend of the data presented here shows a less-than-expected wake growth rate and a higher-than-expected velocity deficit, which would suggest that the spacing of large wind turbines would need to be greater than currently expected to achieve the same array efficiency.

\subsection{RECOMMENDATIONS}

The success in the development and deployment of several visualization methods, in particular the release of smoke tracers from a model rocket ( 1 ine 
source) and from a tether system (point source), has demonstrated emphatically their versatility and field adaptability. It is encouraging that, during the very first deployment last summer, much insight into the wake physics of a MOD-2 wind turbine was gained. Future experiments that can provide important information for the derivation of optimum design and operation criteria for wind farms are recommended. In these experiments, both improved schemes of the methods we developed and other state-of-the-art techniques should be employed.

1. Much of the random error in the velocity deficit and wake height measurements is caused by the inaccuracy of locating the wake centerline relative to the rocket launch pad. Wake meandering and systematic wake curvature are principally responsible for this error, but they are problems which could be circumvented by improving the rocket smoke trail technique. Simultaneous launchings of several rockets spaced evenly across the wake at a single downwind position would allow a two-dimensional mapping of the wake cross section. This method will not require knowledge of the instantaneous wake centerline, since the centerline can be determined from the mapping. Essentially, a wake height contour could be plotted from each simultaneous launch. It is equally plausible to expect a more integrated view of the cross-wake velocity deficit using this approach, since all measured deficits can be referenced to the maximum deficit inferred from the centerline.

Stereo photography may be a more general method to use in such a multiplescale rocket launch, since the velocity can be determined in two dimensions. Both motor-drive cameras could be synchronously triggered as the rockets travel upward so that all the smoke trails are in the view of each camera. The smoke trails could be digitized in a way similar to the present analysis procedure, with the implementation of a new computational algorithm.

2. One of the important wake characteristics that has not been addressed in the present study is the wake width. Knowledge of both the wake height and width will facilitate a complete description of the wake geometry. From the ratio of the wake height to the wake width, we may infer to a certain extent the effect of atmospheric stability and of terrain forcing, 
two of the most important issues. To obtain the wake width, we recommend the release of horizontal smoke trails from rockets guided by a cross-wake cable tethered to two crankup towers. This arrangement will also provide information on the vertical velocity component resulting from convection or terrain forcing. Multiple rockets may be launched simultaneously to map out the wake cross section. In this case, both longitudinal and vertical velocity components can be measured when stereo photography is employed.

3. Further visualization experiments of the tip vortices should be conducted to determine the generation and evolution of the long vortex spouts in terms of their frequency of occurrence, their intensity or energy content, the relative location of their origin, and their trajectories. An attempt should be made to find out how the dramatic vortex reversal is triggered. The specific goal of the experiments should be to provide an estimate of the energy leakage resulting from the escape of the long spouts from the turbine wake. As previously pointed out, appreciable leakage of energy would lead to reduction in wake turbulence, which in turn would slow down the recovery of the momentum deficit in the wake. Consequently, the turbine spacing would have to increase accordingly.

4. To verify our qualitative observation that the wake turbulence was lower than expected, quantitative measurements of the in-wake turbulence intensity are required. In the presence of the wake meander and systematic wake curvature, either a two-dimensional array of point sensors, such as hot-wire anemometers, or an instrument with a rapid scanning capability will have to be used. The deployment of a two-dimensional array of point sensors in such a large-scale operation may not be feasible. However, remote-sensing optical techniques that can scan rapidly, such as laser Doppler velocimeters (LDV), have been developed for atmospheric measurements. For example, a laser velocimeter $\left(\mathrm{CO}_{2}\right)$ is now under development at PNL and a field LDV using a visible laser (argon) has been constructed by Professor F. Durst and his group at Karlsruhe University, West Germany. The latter system has been under extensive calibration and verification in the field for two years. At $106 \mathrm{~m}$, the velocity measurements obtained by the LDV agree well with corresponding results measured by ultrasonic 
flowmeters (Durst et al. 1982). With higher quality optics and higher powered lasers, a range of $500 \mathrm{~m}$ is attainable. However, the 106-m range is more than adequate for turbulence measurements in the wake of a MOD-2 turbine. The use of such laser systems for measurements of wake turbulence should be considered.

5. It is evident that the complex terrain features at the Goodnoe Hills site have a significant effect on the turbine wake. To study the turbine wake characteristics alone, it may be wise to choose another site with relatively flat topographic features. Such a site is now available at Medicine Bow, Wyoming, where a MOD-2 wind turbine and a Hamilton-Standard WTS-4 wind turbine have recently been erected. We recomend that visualization experiments be conducted at this site to circumvent the complication of terrain forcing. In practice, the Goodnoe Hills site represents a real environment in which most wind farms will be operating, because future commercial sites will most likely be in complex terrain. The effect of terrain forcing will, therefore, have to be understood. In that regard, the Goodnoe Hills site is an important asset to the wind energy program. 


\section{BACKGROUND OF THE WAKE PROBLEM}

If wind energy is to provide a significant fraction of a regional electrical energy supply, wind turbines will have to be arranged in clusters, since the land availability, size of the resource areas, and installation costs will favor small spacing between machines. The wakes created by wind turbines favor significant spacing between wind turbines to avoid detrimental effects of one machine upon another. Among the possible aerodynamic effects are diminished resource within the wake, high levels of turbulence, and persistent organized vortices shed from the blade tips. The problem of just how closely machines can be spaced before the negative attributes of close proximity outweigh the positive ones has been and continues to be a high priority problem in the wind energy field. This is especially true today since large numbers of wind turbines are becoming available before the wake problem has achieved a complete solution.

In this section, the background of the wake problem is briefly examined. There is discussion of the critical issues that Flow's flow visualization study addressed.

\section{1 MODELS OF WIND TURBINE WAKES}

A review of the experimental, theoretical, and numerical work that has contributed to the current perception of wind turbine wake structure is thoroughly summarized in Riley et al. (1980). A more abbreviated review appears in Hiester and Pennell (1981). The applicable highlights are summarized here.

A great deal of research has contributed to some concensus on a picture or "mode1" of wind turbine wake structure. This model has been derived from wind tunnel studies, from field data from full-scale wind turbines, and from numerical modeling efforts that have been compared with wind tunnel and field experiments. Wind tunnel studies have used miniature rotors to simulate wind turbines (Builtjes 1979, Vermeulen 1978, Alfredsson and Dahlberg 1979). Wind tunnel studies have also used perforated disks or shrouded tea strainers to create a simulated wind turbine wake (Sforza et al. 1979, Builtjes 1979, Milborrow 1980, Builtjes and Milborrow 1980). Field data from full-scale wind turbine wakes are sparse, but a number of studies have been reported 
(Vermeulen et al. 1979, Faxen et al. 1978, Lissaman 1979, Hansen 1981). Numerical modeling of single turbine wakes has included simple approaches that assume a wake structure and evolution process (Lissaman 1979, Lissaman et al. 1982, Vermeulen and Builtjes 1981, Ainslie 1981) and more complex models that solve the governing dynamical equations of the planetary boundary layer as modified by the presence of a wind turbine (Sforza et a1. 1979, Crafoord 1979). The background from previous research in four elements of wake structure will be highlighted here: velocity deficit, wake geometry, turbulence characteristics, and rotor tip vortices.

First, we present an extremely brief synopsis of the above-mentioned research. Wind turbines are known to modify the flow (thereby creating a wake), and the wake may be described as consisting of a near wake region and a far wake region. The wind turbine retards the approaching flow, extracts kinetic energy and momentum from the mean flow, causes drag which extracts momentum from the flow, and converts kinetic energy of the mean flow into turbulence kinetic energy and energy in organized vortices shed from the blade tips. The near wake region is considered to be highly complex and rapidly evolving. However, many researchers have considered understanding the details of the near wake to be of small importance since it is believed that wind turbines will not be sited so closely as to put one in the near wake of another. It is believed that if far wake structure can adequately be predicted or understood, this is a sufficient solution to the wind turbine spacing problem. The far wake is a region where the wake structure is self-similar. That is, the shape of the mean velocity or turbulence intensity profiles remains constant with increasing downstream distance; only the magnitude of the wake disturbance changes. Thus, apart from the initial momentum extraction, which sets the initial conditions for the absolute structure of the far wake, the far wake is assumed to have no memory of the flow or processes in the near wake.

\subsubsection{Velocity Deficit}

Much of the velocity deficit research results has been presented in terms of the centerline velocity deficit. Field data (Hansen 1981, Vermeulen et al. 1979), wind tunnel data using miniature rotors of the horizontal axis type (Vermeulen 1978, Alfredsson and Dahlberg 1979) and of the Darrieus type 
(Boschloo 1977, Builtjes 1979), and porous disk simulators (Builtjes and Milborrow 1980) all point to similar behavior. In the near wake, the decay rate of the centerline velocity deficit is relatively small. Much of the wind tunnel data show virtually no decay of the centerline velocity deficit, whereas much of the field data just show the decay rate to be less than in the far wake region. In the far wake region, most of the data are consistent with a decay rate proportional to $(x / D)^{-1.25}$, where $x$ is downstream distance and $D$ is rotor diameters. The magnitude of the initial velocity deficit in the near wake is determined by the thrust loading on the turbine (or simulator), whereas the length of the near wake region (the distance to the beginning of the -1.25 decay rate region) is apparently largely controlled by the ambient turbulence intensity and turbulence generated by the rotor.

The validity of using wind tunnel data to describe the near wake behind a real wind turbine is in question. The porous disk simulators clearly do not produce the same wake characteristics in the near wake as a wind turbine. The porous disk does not extract energy. It converts mean flow kinetic energy into turbulence. Wind tunnel models that use miniature rotors also do not adequately represent real wind turbines. The power coefficient of these small rotors is often a function of Reynolds number, is often lower than that of a fullscale wind turbine, and the initial momentum deficit behind these rotors is often larger than that expected for a ful1-scale turbine. All of this indicates that the rotor is operating in stall conditions at least some of the time (Riley et al. 1980). Nevertheless, the wind tunnel data have shown some near wake features that agree to some extent with theoretical expectations, and the associated far wake data have compared favorably with simple numerical models and limited full-scale data. Furthermore, though the near wakes of vertical and horizontal axis turbines are expected to be different in many respects, the far wakes of two miniature rotors of this type were found to be similar.

The slow decay rate of the centerline velocity deficit in the near wake lends support to the idealized actuator disk description of the wind turbine wake. In this description, the initial velocity deficit just behind the rotor is uniform and forms the upstream portion of the potential core. Downstream, the core is gradually eroded away by turbulence generated by shear at the 
boundary of the wake and by turbulence generated by rotor effects. Within the core itself, the velocity deficit remains constant. Therefore, if the potential core description holds some validity, one would expect a constant centerline velocity deficit in the near wake region. The potential core idea is generally regarded as a way to simply describe the near wake sufficiently to set the initial conditions for the far wake and not as a real description of the processes in the near wake. A real wind turbine rotor of low solidity is expected to impart periodicity and swir1 into the flow that cannot be accounted for with actuator disk theory. Wake swirl is known to be small but wake velocity periodicity has been observed (behind a $0.36-\mathrm{m}$-dia rotor) as far downwind as 5D (Vermeulen 1978). The measured cross-wake profile of the velocity deficit is typically a bell-shaped curve, both in the near and far wakes. If there is indeed a region resembling a potential core in the near wake, the cross-wake profile would be expected to be top-hat shaped, with a region of constant velocity deficit around the center of the wake (the potential core), with the velocity deficit decreasing smoothly to zero at the wake edge. It takes a fair amount of imagination to see evidence of the top-hat signature in the wind tunnel data and especially in the field data. of course, these data represent time averages of a wake that is meandering back and forth to some degree, especially in the field. Therefore, time-averaged sampling of a tophat structure that moves in and out of the sampling area will result in a more bell-shaped cross-wake velocity deficit profile. Since the extent of the meandering is greater farther downstream, the time-averaged sampling of a top-hat structure would also result in a slight decay rate of the centerline velocity deficit (as opposed to no decay) that is observed especially in the field data.

The discussion in the preceding paragraph points out an important consideration in the interpretation of wake measurements and wake models. The properties being represented can depict a time-averaged condition of a very mobile wake or can be of a more instantaneous nature. Likewise, the timeaveraged wake effects upon downwind turbines must be distinguished from the effects of the instantaneous wake structure. 


\section{1 .2 Wake Geometry}

Wake radius measurements typically result from measurements of the crosswake velocity deficit profile. The rate at which the wake radius grows is of interest because fast wake growth implies fast mixing of higher momentum ambient air into the wake, which, in turn, infers quick recovery of the flow field. In this discussion, the near wake is defined as $x \leq 1 \mathrm{D}$, and the far wake as $x \geq 4 \mathrm{D}$.

Raw data on the growth rate of wakes is not easily derived from the published literature. Vermeulen (1980) presents wake growth data from virtually all of the wind tunnel experiments available to date. Vermeulen presents these data as part of an argument leading to improvement of the Lissaman (1979) numerical model, which will be described later. The Lissaman model argues that the wake growth near the turbine is due to turbulence generated by the rotor, by shear at the boundary of the wake, and by the ambient turbulence. Vermeulen presents a plot of the component of the wake growth rate attributed to the ambient turbulence intensity versus ambient turbulence intensity. This "measured" growth rate was determined from theoretical equations for total wake growth and near wake length, which are functions of growth rate due to ambient turbulence, rotor-generated turbulence, and shear-generated turbulence at the wake edge. Total growth rate and near wake length were measured. Rotor- and shear-generated turbulence were calculated from theoretical expressions, which were supported by measurements in very low ambient turbulence conditions. The "measured" growth rate due to ambient turbulence, a, is

$$
\left(\frac{d R_{w}}{d x}\right)_{a}=2.5 \alpha+0.05
$$

where $R_{w}$ is the wake radius and $\alpha$ is the turbulence intensity, defined as the standard deviation of longitudinal velocity divided by the mean velocity. In an intermediate distance downstream (5D $<\mathrm{x}<10 \mathrm{D})$, Vermeulen accepts the observed centerline velocity deficit decay rate proportional to the -1.25 power as the anchor point for wake structure and determines a nonlinear wake growth rate from the assumption of conservation of the momentum deficit and a cross- 
wake velocity deficit profile shape. In the very farthest part of the wake, Vermeulen takes the Lissaman (1979) suggestion that the wake grows as

$$
\frac{\mathrm{dR}_{\mathrm{w}}}{\mathrm{dx}}=\frac{\alpha}{0.51}
$$

Using Vermeulen's data for growth rate due to ambient turbulence versus ambient turbulence, Lissaman et al. (1982) suggest the relationship

$$
\left(\frac{d R_{w}}{d x}\right)_{a}=\frac{\alpha}{0.36}
$$

Not only does this fit the data, but they argue that there is theoretical justification as well.

Alfredsson and Dahlberg (1979) data (as presented in Hiester and Pennell 1981) on cross-wake profiles of turbulence intensity measured in a wind tunnel behind a $0.25-m$ horizontal axis rotor give some indication of wake geometry. These data show for grid-generated turbulence (decaying from $5 \%$ to $3 \%$ in intensity) an average growth rate of 0.25 for $x<4 D, 0.15$ for $4 D<x<8 D$, and 0.06 for $8 \mathrm{D}<\mathrm{x}<12 \mathrm{D}$. The far wake data imply $\mathrm{dR}_{\mathrm{w}} / \mathrm{dx}=\alpha / 0.5$.

Hansen (1981) presents some information on wake growth for the low ambient turbulence intensity $(1 \%)$ case. His data show a rapid growth rate $\left(\mathrm{dR}_{\mathrm{w}} / \mathrm{dx}=015\right)$ for $x<5 D$, but very slow growth $\left(d R_{w} / d x=0.04\right)$ for $x>5 D$. These far wake data imply $\mathrm{dR}_{\mathrm{w}} / \mathrm{dx}=\alpha / 0.25$.

\subsubsection{Turbulence Characteristics}

Turbulence in a wind turbine wake is generated by drag on the nacelle, tower, and blades; by breakdown of rotor tip vortices; and by shear generation in the shear layer between the wake and the ambient flow. Turbulence intensity measured deep in simulated arrays of wind turbines may in fact be the dominant determinant of array design (Builtjes and Milborrow 1980).

Note that the way most investigators have reported turbulence intensity is as the standard deviation of the longitudinal velocity divided by the mean wind speed. In this way, if rotor tip vortices are present, their motion is counted as turbulence, although these vortices technically are organized structures and not turbulence. 
Vermeulen (1978) reports centerline turbulence intensity measurements that show a roughly constant value of 5 to $8 \%$ in the near wake but with an abrupt increase to about 9 to $10 \%$ at $4 \mathrm{D}$ to $5 \mathrm{D}$ or at the beginning of the far wake. He argues that this is consistent with the "potential core" description of the wake, since this behavior indicates that the centerline does not experience the high turbulence generated at the wake boundary until the potential core is eroded away.

Recall from above that Vermeulen (1980) determined the component of the wake growth rate due to ambient turbulence by assuming functional forms for the growth rate due to turbulence generated by the wind turbine and the shear in the near wake. This generation of turbulence is related to the turbine thrust coefficient, $C_{t}$. Vermeulen then argued that the near wake length $\left(x_{n}\right)$, which is assumed to be controlled by this generation of turbulence by the wind turbine, should be a controlling length scale. He plotted data of $\left(u / U_{0}\right)\left(1 / C_{t}\right)$ versus $x / x_{n}$, and found that the data collapsed so that $u / U_{0}=0.13 c_{t}$ at $x=x_{n}$. The decay rate of the turbulence for $x>x_{n}$ depended on the ambient turbulence intensity and was faster for higher turbulence.

The results of Builtjes (1979) are considerably different. His results, which were for a Darrieus model rotor in a somewhat more turbulent ambient flow, show a continuous (power law) decrease of turbulence intensity from $30 \%$ at $2 \mathrm{D}$ to $4 \%$ at $20 \mathrm{D}$.

Vermeulen's (1978) cross-wind profiles of turbulence intensity show a spike just downstream and near the rotor tip, which is associated with the rotor tip vortices. Power spectrum measurements at the point of the spike also show that much of the energy in this spike is associated with the blade passage frequency. This spike spreads out laterally at downstream locations, which again agrees with the Lissaman model of a shear layer with self-generating turbulence that spreads inwards and outwards (Riley et al. 1980). Data of Alfredsson and Dahlberg (1979) (also shown in Hiester and Pennell 1981) show similar behavior.

\subsubsection{Rotor Tip Vortices}

Tip vortices are shed from the ends of rotor blades as a result of the 1 ift on the blades. Not a great deal is known about their generation, persistence, and role in the wind turbine wake processes. Certainly, little full-scale data are available. 
In the wind tunnel, Vermeulen (1978) noted periodicity in the flow at 5D downwind of the rotor. Whether these were tip vortices or turbulent remnants of vortices is not clear.

Alfredsson and Dahlberg(a) observed rotor tip vortices through flow visualization in the wind tunnel. They presented a plot showing a zone of downwind distances $(x / D)$ and longitudinal turbulence intensities within which rotor tip vortices were observed. Tip vortices were observed as far downwind as $4 \mathrm{D}$, but only when the turbulence intensity was less than $2 \%$. At a $10 \%$ turbulence level, the vortices were not observed beyond 1 or $2 \mathrm{D}$.

An unpublished study by the National Aeronautics and Space Administration (NASA) resulted in a motion picture of experiments that released smoke just upwind of the blade tips of the 39-m-dia MOD-0 wind turbine. (b) The films clearly showed the rolling up of the smoke into the tip vortices being shed from the blade. The persistence and the possible role of tip vortices in the wake of full-scale turbines remained in question.

\subsubsection{Practical Models}

Wind turbine clusters are being built now. Most of the siting of wind turbines within these arrays is dictated by topographical and other siting constraints along with general spacing guidelines that have been developed from previous studies. Organizations that are installing these arrays could have more confidence in their siting decisions if there were practical models available that predicted the effects of wakes within an array. In general, these methods are not yet available. Some methods are close to being useable, and, since they are the only tools available, probably will be used in the near future. This section takes a brief look at the options.

Numerical modeling of single wakes is proceeding along several fronts. Most models that use dynamical equations are a long way from being verified. The contribution of some of these models (e.g., Crafoord 1979) has been to point out the significance of certain parameters, such as atmospheric stability. Another approach includes simple algebraic descriptions of wakes, virtually all of which are based on the actuator disk approach.

(a) International Energy Agency Annex $V$, working meeting, Central Electric

(b) Research Laboratories, Leatherhead, U.K., February 2-3, 1981.

(b) T. R. Richards, Personal communication, 1982. 
The first model of this type is due to Lissaman and Bate (1977), and is also described in Lissaman (1979). This model was based on experimental data of Abramovich (1963) on coflowing jets, specifically the case where the inner jet is of lower velocity than the outer jet. According to Abramovich, one can define two distinct regions: the initial region in which a constant velocity potential core exists and a far dowstream region. In the far region, the velocity profile is described by

$$
\frac{\mathrm{u}}{\mathrm{U}}=1-\Delta \mathrm{U}\left[1-\left(\frac{\mathrm{r}}{\mathrm{R}_{\mathrm{w}}}\right)^{1.5}\right]^{2}
$$

where $u$ is the local velocity,

$\mathrm{U}$ is the free-stream velocity,

$\Delta \mathrm{U}$ is the local centerline velocity deficit which is related to the initial velocity deficit,

$r$ is the distance from the inner jet (wake) centerline, and

$\mathrm{R}_{\mathrm{w}}$ is the inner jet (wake) radius at the local downstream position.

The velocity deficit profile in the inner jet is similar to a Gaussian profile, except that a Gaussian profile would have a finite deficit at an infinite radius, whereas the data of Abramovich indicate a discrete wake edge at $\mathbf{r}=\mathrm{R}_{\mathrm{w}}$.

The momentum thickness, $\theta_{m}$, is obtained by the following integral:

$$
\theta_{\mathrm{m}}=2 \pi \int_{0}^{\infty} \frac{\mathrm{u}}{\mathrm{U}}\left(1-\frac{\mathrm{u}}{\mathrm{U}}\right) \mathrm{r} \mathrm{dr} .
$$

In the Lissaman model it is assumed that this is a constant throughout the whole evolution of the wake; i.e., the momentum deficit is conserved. Thus, Lissaman has a relationship between the velocity profile and the wake radius. With a description of the initial velocity deficit, and one for the change in wake radius, the problem is closed. The initial velocity deficit is obtained from actuator disk theory, which provides:

$$
\frac{u(x=0)}{U}=\left(1-c_{t}\right)^{0.5}
$$

where $C_{t}$ is the turbine thrust coefficient. Lissaman assumed the growth rate 
in the near wake region is due to the combination of shear-generated turbulence at the wake boundary $\left[\left(\mathrm{dR}_{\mathrm{w}} / \mathrm{dx}\right)_{\mathrm{m}}\right]$ and ambient turbulence entrainment of the wake. The shear-generated portion was described in equations by Abramovich. Citing growth of Gaussian plumes of scalar contaminants, issaman suggests the growth rate due to ambient turbulence is given by $d R_{w} / d x=\alpha / 0.51$, where $\alpha$ is the longitudinal turbulence intensity. Lissaman (1979) then argues that the scale of turbulence in these two growth processes is similar, i.e., the growth pro cess is governed by the sum of the turbulence from the two sources. Thus, the overall growth rate is a Pythagorean addition of individual growth rates as:

$$
\frac{\mathrm{dR}_{\mathrm{w}}}{\mathrm{dx}}=\left[\left(\frac{\mathrm{dR}}{\mathrm{dx}}\right)_{\mathrm{m}}^{2}+\left(\frac{\mathrm{dR}_{\mathrm{w}}}{\mathrm{dx}}\right)_{\mathrm{a}}^{2}\right]^{0.5}
$$

Where the wake impinges against the ground, imaging techniques are used similar to problems in transport of scalar contaminants. A virtual wind turbine is created in mirror image fashion beneath the ground plane. The velocity deficits of the wake and the image wake are added together where the wakes intersect above the ground plane. This conserves the velocity deficit above the ground plane.

The Lissaman model has spawned a number of derivative models. Vermeulen (1980) has suggested improvements to the model that reportedly make the model fit wind tunnel and 1 imited field data better. Vermeulen felt that the Lissaman model overpredicted the length of the near wake region, and that the reason for this was that the model neglected the generation of turbulence by the rotor itself, i.e., viscous rotor drag and tip vortices. Vermeulen incorporated changes based on his analysis of the data. Vermeulen also felt the data clearly indicated a centerline velocity deficit proportional to $\mathrm{x}^{-1.25}$ beyond the near wake but perhaps before the very far wake. So he assumed this centerline velocity deficit, the conservation of momentum deficit, and the cross-wake velocity profile of Abramovitch to derive the wake radius and hence the growth rate. In the very far wake, he assumes the growth rate due to ambient turbulence alone, and assumes the expression given by the Lissaman model. A model very similar to Vermeulen's has been created in the United Kingdom (Ainslie 1981). 
With additional wind tunnel results available, the Lissaman model has been improved in the United States (Lissaman et al. 1982). One change resulted in the addition of an expression for the growth rate component due to the rotor effects. An expression for the loss of energy due to drag on the rotor was derived. It was acknowledged that not all of the energy lost would result in turbulence, but that some of it would result in organized tip vortices. It was also recognized that the scale of this turbulence might not be such that the Pythagorean addition of wake growth rates would be permissible. These two factors were accounted for via a proportionality constant. The constant was chosen to agree with wind tunnel data.

Another change in the original Lissaman model was the change in the proportionality constant in the growth rate due to ambient turbulence, from 0.51 to 0.36 , so that in the far wake

$$
\left(\frac{d R_{w}}{d x}\right)_{a}=\frac{\alpha}{0.36}
$$

where $\alpha$ is the standard deviation of the lateral wind component divided by the mean wind. The above expression is argued to have theoretical merit. It assumes the expression used to describe growth of Gaussian plumes of scalar contaminants, but with the Gaussian plume dispersion width replaced with a similar factor derived from the Abramovitch profile. The factor was derived by equating the integrals of the Abramovitch profiles and the Gaussian function.

Lissaman et al. (1982) compare the improved model with the wind tunnel data as best they can. They make a good point however, in pointing out that little of the wind tunnel work uses a simulated boundary layer. Instead, much of it is done using grid-generated turbulence. Grid-generated turbulence decays, thus, it is argued, the local ambient turbulence is decreasing downstream, contributing to a less-than-natural wake growth rate.

These models are simple to implement and use for array modeling. For this reason, if any wind turbine array model is used in practice in the near future for siting decisions, it will likely be one of these or one very much like them. What the above models do not yet do is operate in complex terrain. To practically obtain wind turbine array design input for complex terrain, the likely method will be to use porous disk simulators in wind tunnel models. 
Wind tunnels also have limitations in their ability to simulate flow over complex terrain (Hiester and Pennel1 1981).

\subsection{CRITICAL ASSUMPTIONS TO BE TESTED}

We have, at the present time, the following choices for tools to use in modeling arrays of wind turbines. First and most simple are the numerical models of the momentum conservation type. These have been tuned with wind tunnel data and limited field data, but extensive comparisons with arrays or even single large machines are not available. Second are the physical models of arrays using wind tunnel simulators. These simulators provide theoretical and empirical support of the simple numerical models but, again, field data are lacking to verify with confidence that the simulators are sufficiently accurate. Third, we have rules of thumb. Most of the rules of thumb that have been suggested are based on the wind tunnel and simple numerical model results. A great deal of research has been done, yet the immediacy of the problem calls for some examination of the critical assumptions that are implicit in the models and methods that are likely to be used. This section identifies the assumptions that our study has attempted to address.

\subsubsection{Velocity Deficit Profile}

All existing data point to a bell-shaped or quasi-Gaussian shaped velocity deficit profile in the far wake. This study has examined the velocity deficit profile in detail. Most previous examinations of this profile shape have been time averages. This naturally results in a bell-shaped curve since there is averaging of the intermittency at the edge of the wake (Tennekes and Lumley 1972). The intermittency is due to the detailed structure of the entrainment process plus meandering of the entire wake caused by very large structures within the planetary boundary layer. The profile shape of the time-averaged velocity deficit is appropriate for gross estimates of wind array energy pro duction, but the instantaneous wake velocity (and turbulence) structure is what is seen by downstream wind turbines. Therefore, this study attempts to look at the velocity profile shape in an attempt to qualify the quasi-Gaussian profile shape assumption, plus provide new information on the instantaneous profile shapes. 


\subsubsection{Wake Geometry}

Wake geometry is one measurable quantity that has the potential to substantially verify the assumptions currently being made about wind turbine wake behavior. If the wake grows rapidly, then through conservation of the momentum deficit, the wake centerline velocity deficit will fall off quickly, encouraging the possibility for closer wind turbine spacing. Conversely, if the wake grows more slowly, then the velocity deficits could be expected to be more severe. In either case, if measured MOD-2 wake geometry does not fit with expectations, there will be an indicated need to revise the assumptions of the models upon which those expectations are based.

\subsubsection{Wake Turbulence Characteristics}

It has been suggested that wake turbulence might dominate the intermachine spacing criteria. Wind tunnel models that are designed to extract a certain fraction of the mean flow energy do so by converting mean flow energy into turbulence. Does this modeling process bias the conclusions about wake spacing in any way? This study visualizes certain flow characteristics that can indicate whether or not wake turbulence behaves as the wind tunnel models suggest.

\subsubsection{Role of Rotor Tip Vortices}

The generation and persistence of rotor tip vortices in large-scale wakes have received little attention prior to this study. How far downwind do the vortices persist? Do the rotor tip vortices contribute to the turbulence in the wake (and influence wake growth) as described in the simple models? This study sheds light on these questions. 


\section{EXPERIMENTAL STRATEGY AND EXECUTION}

This section describes the site chosen for the experiments and the overall experimental strategy. It concludes with a sumary of the execution of the field experiments.

\subsection{THE GOODNOE HILLS SITE}

The site chosen for these wake studies is the MOD-2 cluster at Goodnoe Hills, near Goldendale, Washington. In October 1979, the Department of Energy (DOE) selected the Bonneville Power Administration (BPA) to be the operator of the first three MOD-2 wind turbines (Axell and Woody 1981); BPA had proposed the Goodnoe Hills site. One of the main objectives of the MOD-2 wind turbine pro ject is to provide the necessary ful1-scale environment for gathering pertinent field data that will aid in the evaluation of the interactive and machine/grid effects of multiple, identical machines integrated into a utility network. There are three MOD-2 wind turbines at that site (Figure 4.1).

The Goodnoe Hills site is atop a 760-m ridge oriented approximately eastwest. There is a steep dropoff to the Columbia River to the south, and a somewhat gentler dropoff to the north and west to the Klickitat Valley. Juniper Point, a peak of $954 \mathrm{~m}$ is the next significant topographical feature $13 \mathrm{~km}$ to the west-southwest along the same ridge chain. The prevailing winds are from the west through northwest. Spring and summer are the principal wind seasons at Goodnoe Hills. Pressure gradients that form across the Cascade Mountains, which separate cool marine air from hot inland desert air, drive air up the Columbia River Gorge and up over the Goodnoe Hills. The winds from this seasonal mean pressure gradient are modulated by the passage of cyclonic lowpressure systems or high-pressure systems that can either temporarily cut off the flow through the gorge or enhance it. There is also a typical diurnal oscillation that causes winds to be light and southerly in the mid-morning, increasing to westerly winds as daytime mixing proceeds, to strong westnorthwest winds by evening as winds responding to the large-scale pressure gradient reach their peak. The winds continue strong from the northwest throughout the night at ridgetop level where winds accelerate in response to decoupling from the friction at the earth's surface brought on by nighttime cooling. 


\section{GOODNOE HILLS, WA}
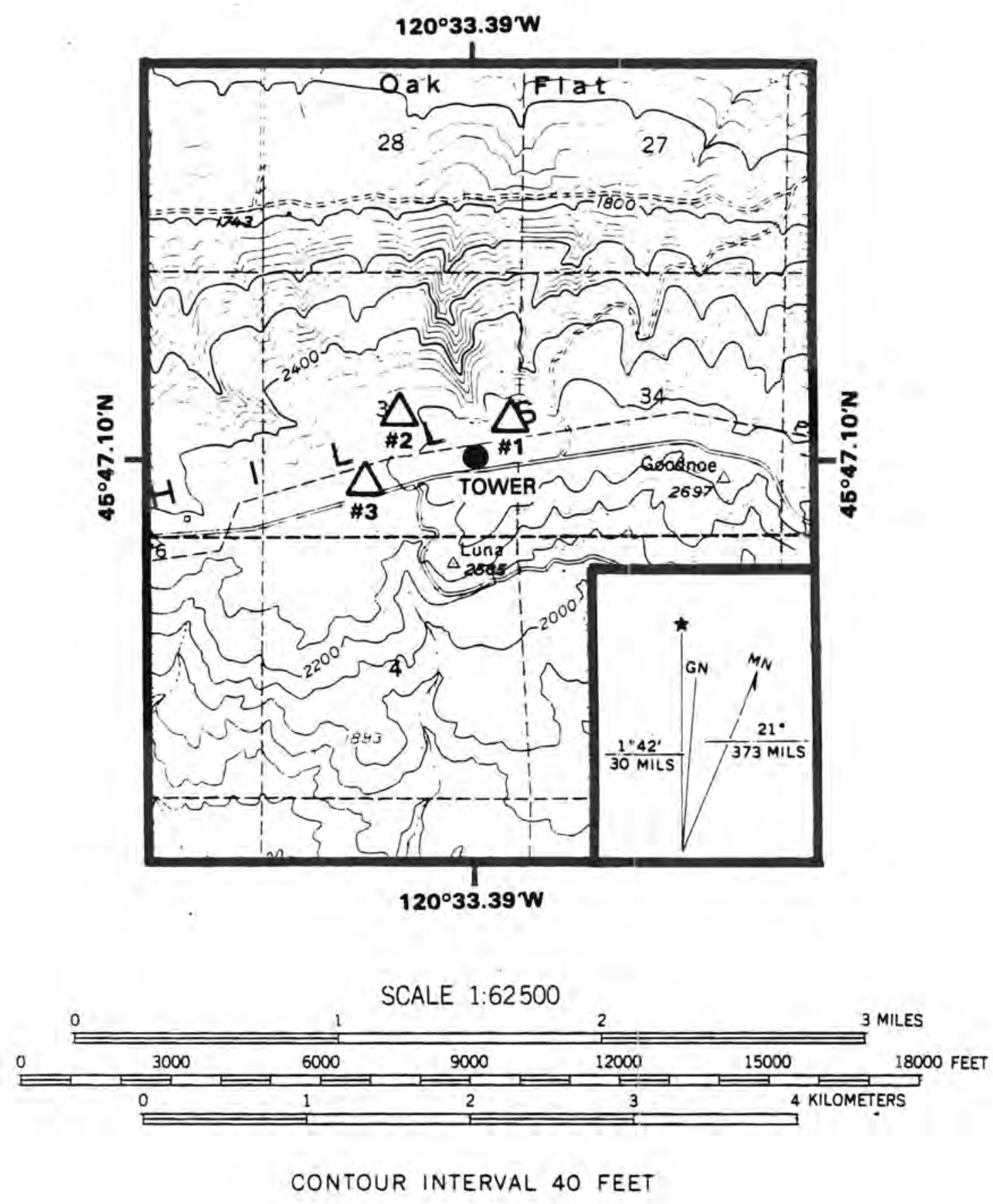

Figure 4.1 Goodnoe Hills Test Site 
The MOD-2 cluster was 1 aid out to study the wake effects of one turbine upon another. Figure 4.1 shows the locations of the three turbines and the 107-m PNL tower. Not shown is the 60-m BPA meteorological tower located approximately one-third of the way from turbine 非 3 to 非2. The separation between turbines 非 1 and 非 2 is 7D, while the separation between 非 1 and 非 3 is $10 \mathrm{D}$, both in a direction to frequently align two turbines with the wind. Turbines 非 2 and 非 3 are $5 \mathrm{D}$ apart in a direction that experiences relatively few occurrences of strong winds. The site, though it appears relatively flat, is not simple by micrometeorological standards. There is a $16-\mathrm{m}$ difference in elevation between units 非 1 and 非. A ravine cuts southeastward into the site between units 非 2 and 非. About $1 \mathrm{~km}$ upwind of unit 非3, there is a forested slope.

Routinely acquired site data used in this project were collected on the MOD-2 Project Distributed Data System, operated by PNL. The system collects and stores data in user selectable rates and formats from the 10- and 59.4-m levels of the BPA meteorological tower, from the 10-, 15-, 61-, and 107-m levels of the PNL meteorological tower, and from eight channels from each of the three wind turbines.

\subsection{EXPERIMENTAL STRATEGY}

There was only 1 imited time available for this project, which began in June and ended in September. The emphasis was placed on determining the wake characteristics of a single wind turbine that is not significantly influenced by other turbines. Wind turbine 非 3 was the only turbine used in this project. The selection was based on the following considerations:

1. Under the prevailing wind conditions, the wake behind 非 3 extends up to 10D with little interference from the other two wakes.

2. Nearby Highway 8 facilitates the transport of equipment along the entire length (up to 10D) of the wake axis.

3. All equipment, except cameras, can be operated from within the MOD-2 site boundary.

To achieve the objectives of the proposed work, several methods of flow visualization were tested and used. The strategy of method selection provided for an overlap among methods to facilitate cross-comparison and evaluation of methods and results. The methods range from tracers, which show the wake 
evolution in the Lagrangian perspective, to tracers made visible in fixed planes, which provide an Eulerian view of the wake time history. Some of the methods have been successfully applied in atmospheric tests of scales similar to the MOD-2 project. A few of the methods were newly developed, based on the authors' laboratory experience in physical modeling using a tow tank. Some of the methods worked well and will provide important tools for future turbine wake studies and for atmospheric flow investigations. Other methods did not work as planned in the field. Still other methods did not receive adequate testing on this project because they were designated as low priority tasks which did not get completed.

The most successful techniques turned out to be the observation of vertical smoke trails laid down by model rockets, observation of smoke from a smoke generator placed just downwind of the rotor, and observation of streamers attached to the tether of a large parafoil kite. Details of the most successful methods are presented in Section 5 .

The experimental strategy was to attempt to obtain wake data in a variety of conditions, yet not under such a wide variety that there was no hope of achieving repetition for any given condition. First, it was desired to obtain information from wind speeds between 7 and $12 \mathrm{~m} / \mathrm{s}$. Within this range of wind speeds, the power coefficient of the rotor of the MOD-2 is between 0.32 and its maximum value of about 0.37 . It is in this range of power coefficients that one expects to be able to best observe wake effects, since the velocity deficit (either absolute or normalized by the free-stream winds or both) is largest.

Second, it was desired to obtain infotmation from both unstable and stable atmospheric stability conditions. Under unstable conditions, it is expected that turbulence and momentum transfer would be greatest, thereby contributing to rapid wake decay. Conversely, it would be expected that under stable conditions where turbulence is suppressed, wake growth would also be suppressed. It was not clear what to expect of the rotor tip vortices in stable conditions, since one argument claims that the vortices would persist longer due to the suppression of turbulence, and another argument claims that the vertical motions of the vortex action would likewise be suppressed and vortex instability would be promoted (Maxworthy 1977). The criterion for defining unstable versus stable conditions was based on the sign of the potential temperature gradient. 
The third condition that had to be met was that the wind direction was greater than $240^{\circ}$ but less than $280^{\circ}$. These were the prevailing wind directions and also were the directions with the simplest terrain downwind of turbine 3 . Finally, measurements were sought in the "near wake" and the "far wake". For this purpose, the far wake was defined as points at 4D or beyond.

\subsection{EXECUTION OF EXPERIMENTS}

The experiments began with atmospheric testing of various techniques and methods at Flow's headquarters in Kent, Washington. There, the rocket design (see Section 5.1.1) was tested and modified. The visibility of smoke and the photographic techniques (exposures, film speed, filter combinations etc.) were tested. Balloon release and tracking techniques were also verified and practiced.

One proposed test was a smoke test with illumination from a searchlight. The purpose was to enable flow visualization during strong nightime wind conditions when the atmosphere was stably stratified. The technique was tested in Kent and found to be workable. However, the searchlight technique became of secondary priority as the field experiment progressed, and was never used at Goodnoe Hills.

Once all preliminary tests were completed at Kent, the experimentation moved to Goodnoe Hills. Three study periods of five to eight days in duration were projected at the beginning of the project. The first period was to be a shakedown of all the techniques under actual field conditions. The second period was to concentrate on the near wake and unstable conditions. The third period was to emphasize the far wake and stable conditions.

Due to several factors, the data base obtained during the sumner experiments was somewhat less than what was planned. Development of the rocket smoke trail technique to a point in which the success rate was greater than $90 \%$ spanned the first two test periods. Rocket engine and smoke candle malfunctions delayed full utilization of the technique until late in the project. It was determined by the rocket engine manufacturer that Flow had received a shipment with a high percentage of defective rocket engines. This should be regarded as an unfortunate coincidence, since these rocket engines have a statistically high reliability record. The smoke candle malfunction was 
tracked down to smoke candles that became overheated while in storage at the Goodnoe Hills between the first and second test periods. Once diagnosed, that problem could be avoided. Finally, a lack of wind during the second and third test periods truncated some of the planned rocket and other experiments. To compensate, two additional test periods were added for a total of five field periods. 


\section{EXPERIMENTAL METHODS}

Several experimental methods employing different tracers were used to aid in visualizing flow characteristics of the wind turbine wakes. The visualization experiments were recorded on both still and movie films. From the results of flow visualization, we derived qualitative as well as limited quantitative information for understanding essential properties of the wind turbine wakes. In this section, we decribe the various experimental methods and the pertinent processes of data analysis.

\subsection{METHODS OF FLOW VISUALIZATION}

After a series of small-scale and onsite feasibility testings, we selected several promising methods to visualize the flow characteristics of the wind turbine wakes. The visualization methods included three types of tracers: smoke tracers, neutrally buoyant balloons, and streamers. The same tracers were used for visualizing different wake characteristics, such as vertical profiles of the longitudinal velocity, rotor tip vortices, and wake heights; but the methods of deployment varied depending on the flow phenomena of interest. In the following, we describe the methods of flow visualization used for observing the various wake characteristics.

\subsubsection{Vertical Profiles of Longitudinal Velocity}

To obtain a vertical profile of the longitudinal velocity, time-lapse photographs were taken of vertical smoke trails released from smoke generators mounted on an upward traveling model rocket moving at about $80 \mathrm{~m} / \mathrm{s}$. (a)

Figure 5.1 gives a conceptual drawing of the experiment. The rocket includes a nose cone, a payload section for the smoke generators (four 1-min orange smoke candles ${ }^{(b)}$ ), a rocket body in which a parachute is placed, a set of three fins, and an E-size rocket motor at the tail section. A detailed description of the rocket design and assembly is given in Appendix A. Figures 5.2 and 5.3 are a sketch and the corresponding photographs of the rocket assembly, respectively. The rocket assembly was placed in a launching pad with an electronic

(a) The original design of the model rocket was provided by Jim Konarski, a model rocketeer, and was subsequently revised by Flow's personnel.

(b) Manufactured by E. Vernon Hil1, Inc., P.O. Box 14248, San Francisco, CA 94114. 31 


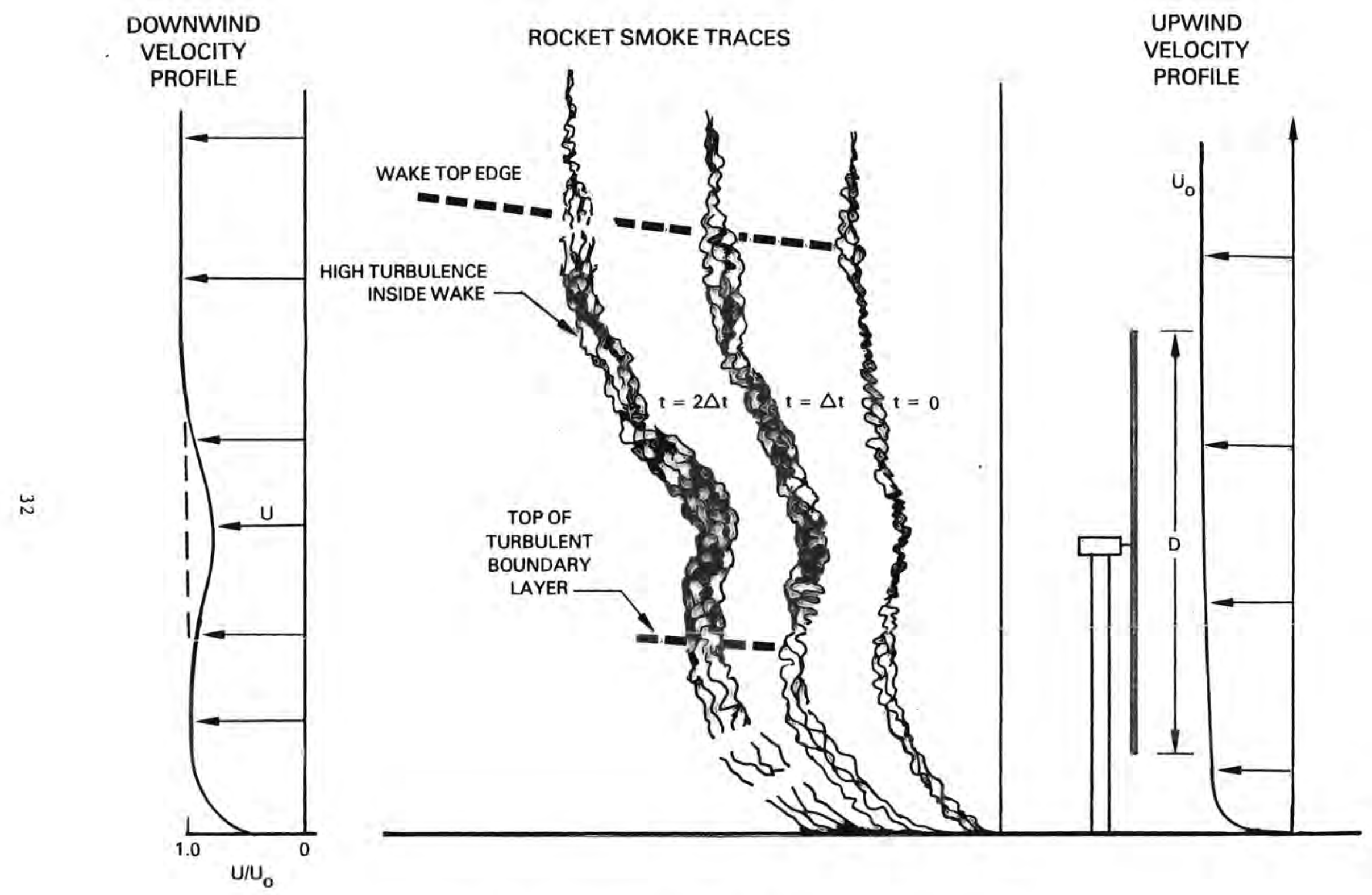

Figure 5.1 Conceptual Sketch of Rocket Trail Experiments 


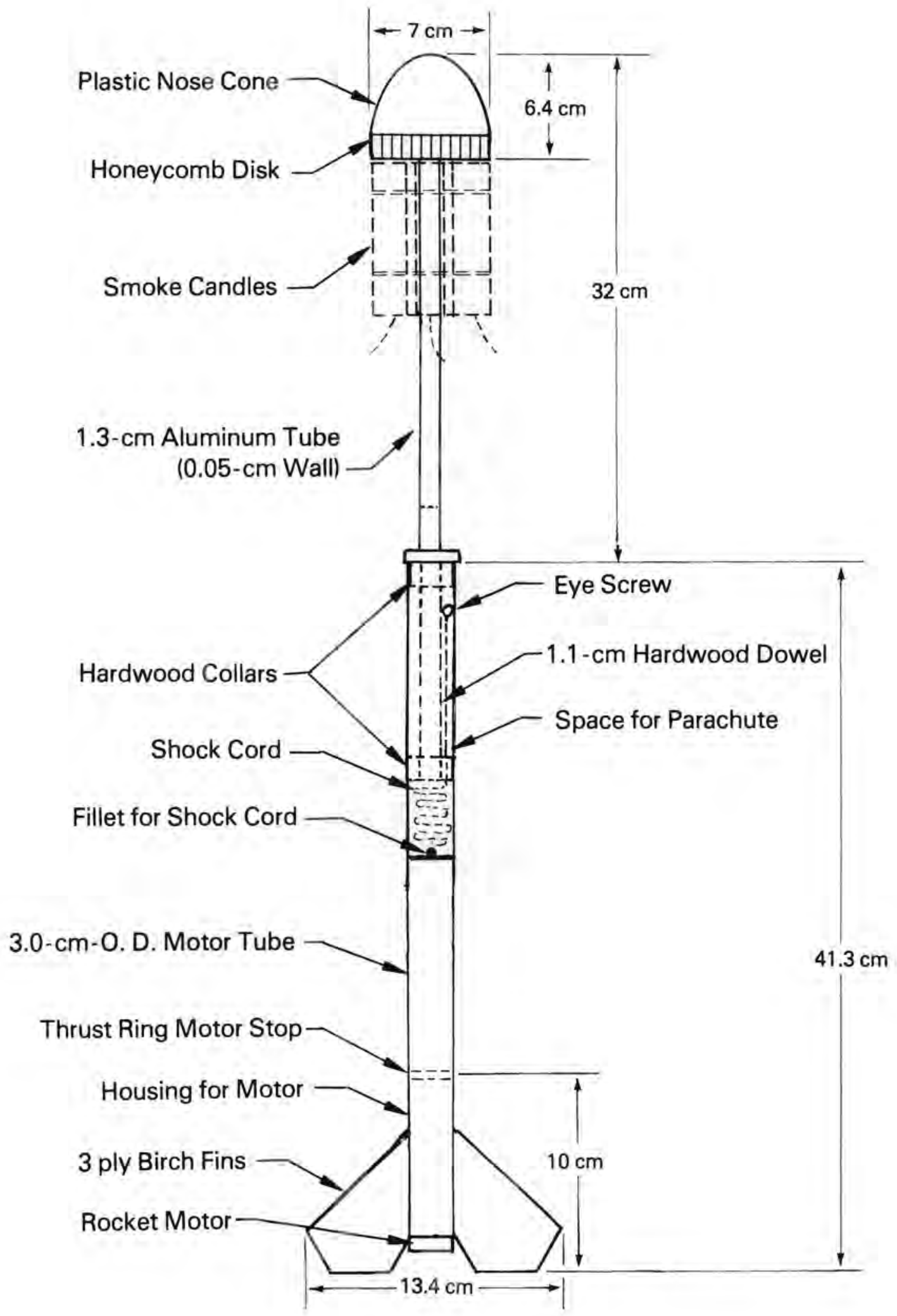

Figure 5.2 Sketch of Rocket Assembly 

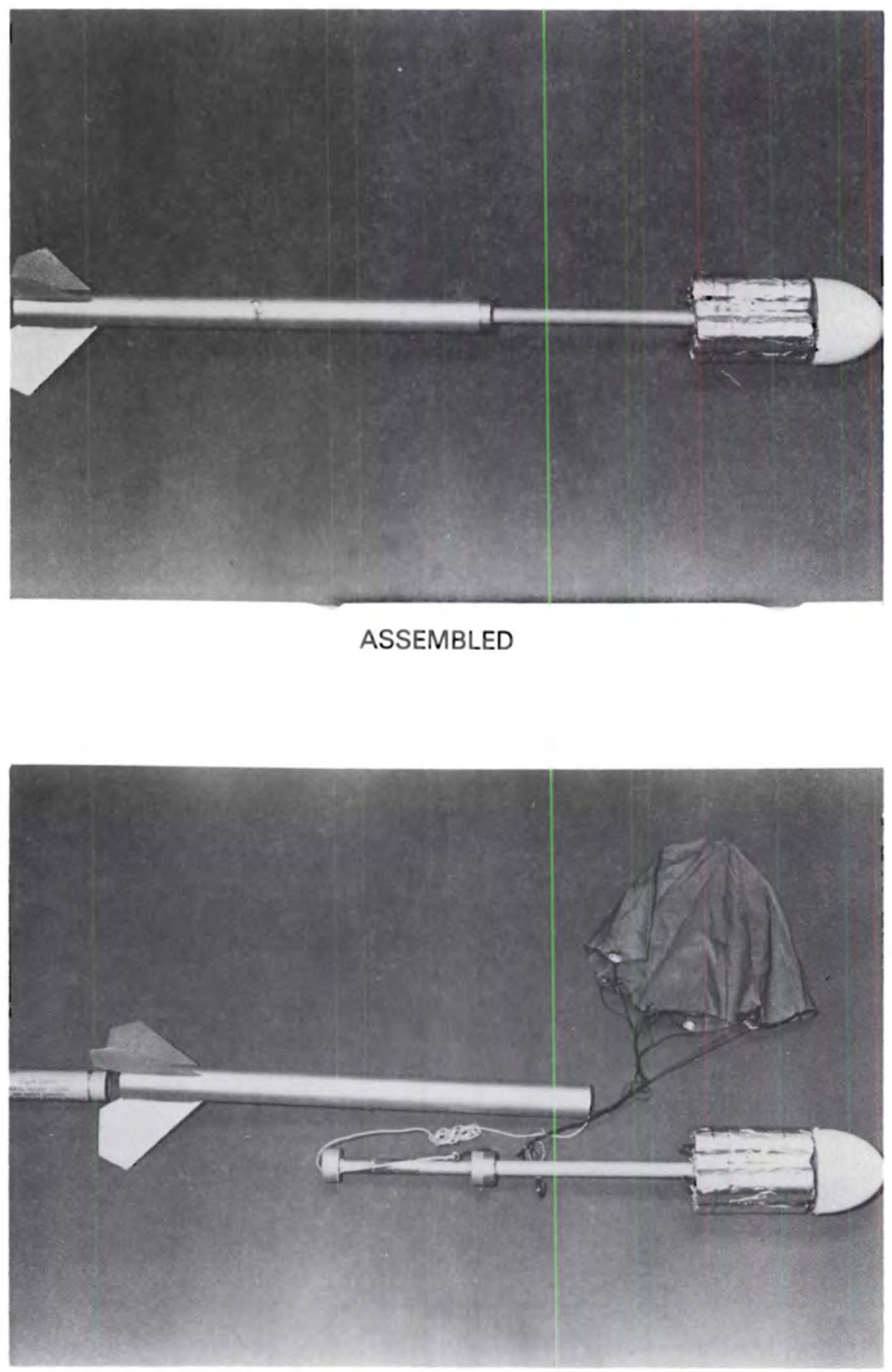

DISASSEMBLED

Figure 5.3 Photographs of Rocket Assembly 
ignitor placed inside the rocket nozzle. The ignitor was set off by using a 12-volt car battery. Before ignition, the smoke candles were 1 it for about 30 seconds until all four candles were burning at full strength. The smoke released from the candles leaves a nearly vertical smoke trail along the path of the rocket. At the end of the vertical traverse, a delayed charge is fired to release the parachute placed inside the rocket body. The parachute slows down the speed of descent of the rocket assembly for ease of recovery. A person equipped with a fire extinguisher was assigned to recover the rocket. He was positioned downwind at the point of expected impact and his sole responsibility was to recover the rocket assembly.

The rockets were launched at downwind points along an anticipated wake centerline and viewed from cameras placed perpendicular to the centerline. Two $35-\mathrm{mm}$ motor-drive cameras recorded the rocket trail advection at a rate of either two or four photographs per second. Depending on the turbulent intensity, the persistence of the smoke was from about 3 to 15 seconds. Typically, a photographic record of 18 consecutive slides is available for each rocket launch or about one-half of a 36 exposure roll of slide film. Figure 5.4 shows the generalized geometry of the rocket experiments. The angle $\theta_{\text {eff }}$ is defined as the effective turbine axis angle and is the deviation between the wake centerline and the orientation of the baseline on which the rocket is launched. A summary of the site geometry laid out behind turbine 非 3 is presented in Figure 5.5.

Prior to the launch of a rocket, a decision was made to set the launch pad on a turbine axis baseline corresponding to the mean expected advection of the wake centerline. Cameras were placed at the corresponding off-axis camera station. The rocket was then launched and retrieved. Since the wind direction during the launch from both meteorological towers was recorded at a 5-sec sample interval, the mean advected centerline of the wake can be determined. In actuality, onsite determination of the position of the wake centerline was difficult, and thus it was a rarity to launch a rocket very close to the centerline. With multiple rocket launches at a variety of off-axis angles from a single downwind position, a reconstruction of the two-dimensional wake was deduced from the rocket launches. 


\section{LEGEND \\ $X$ ROCKET LAUNCH PAD \\ O camera STATION}

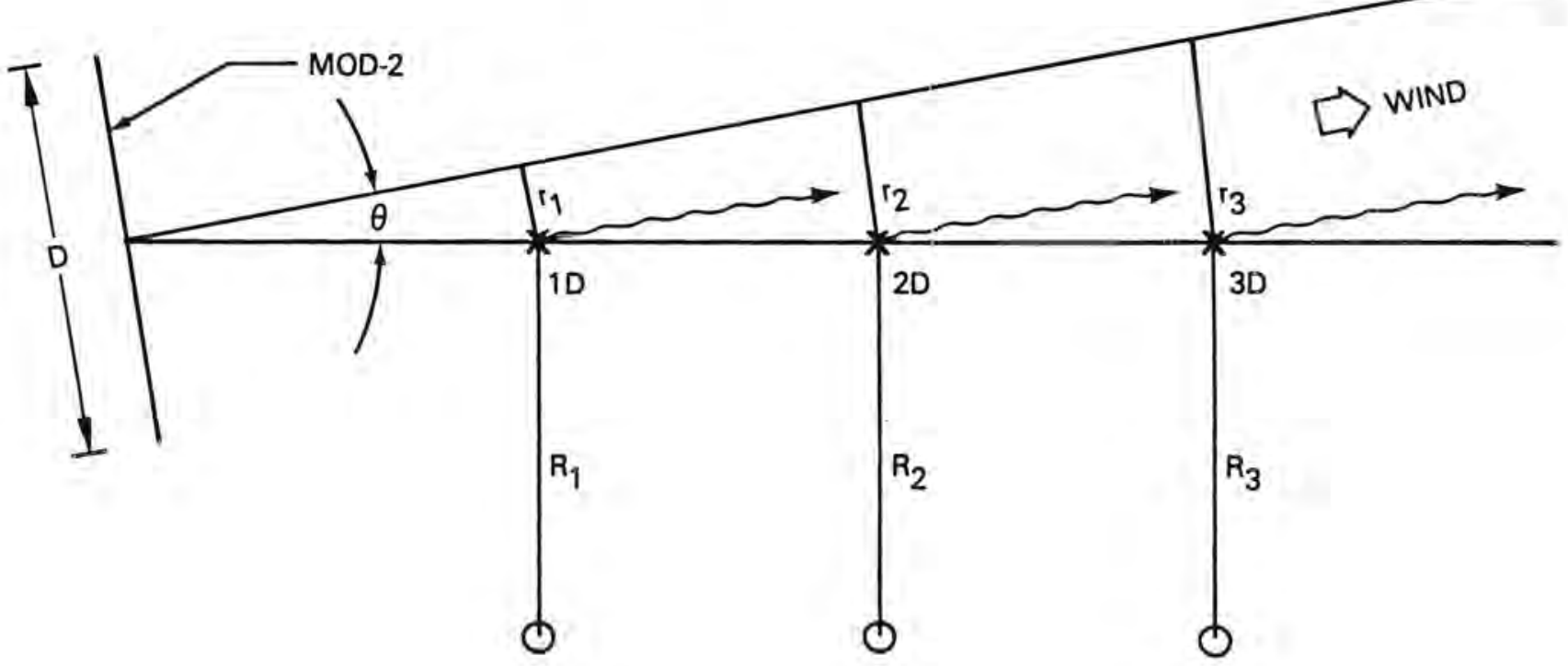

Figure 5.4 Geometrical Layout of Rocket Experiments 


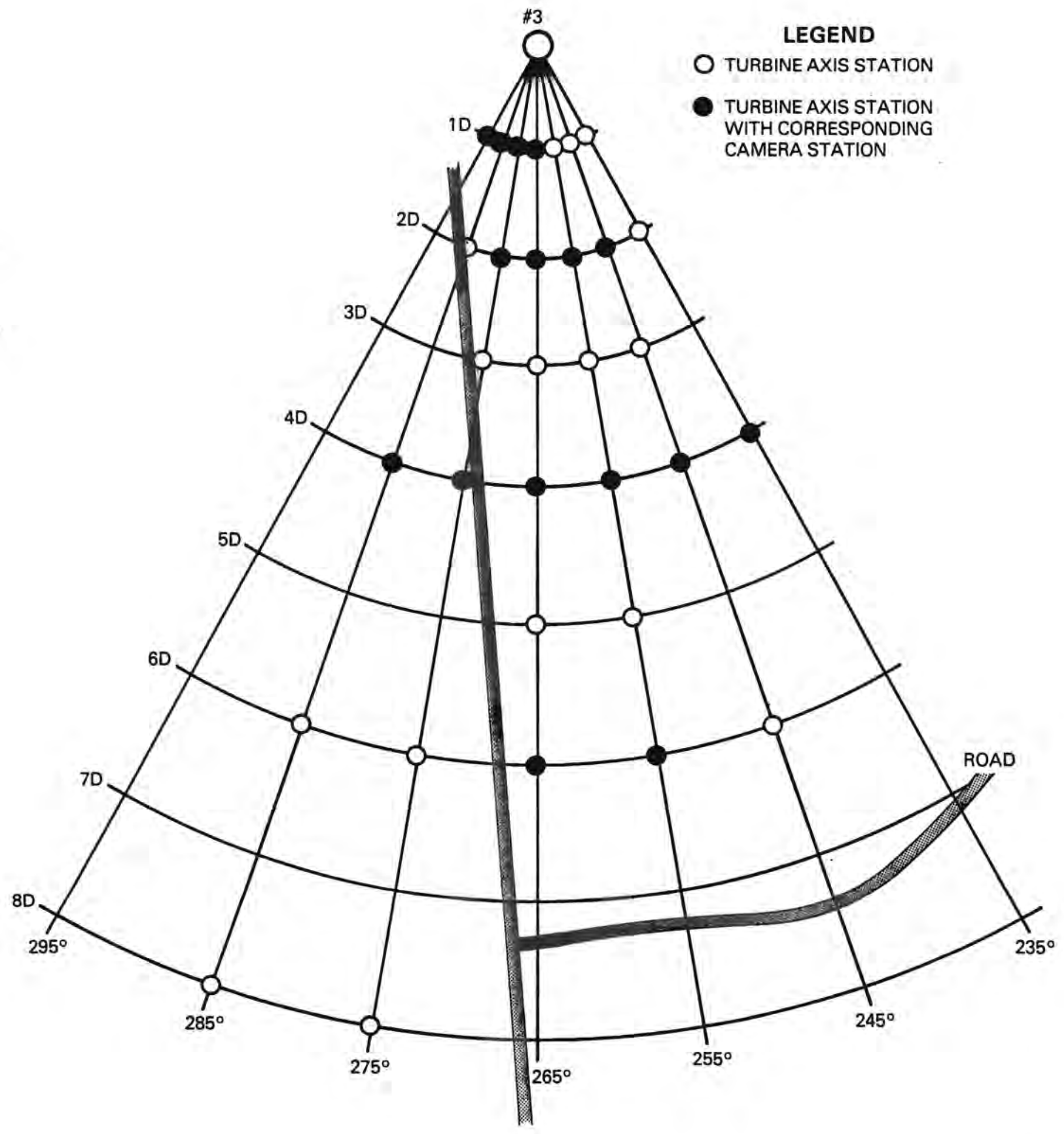

Figure 5.5 Placement of Stations Downwind of Turbine \#3 
The velocity profile as deduced from the smoke trails is an instanteous "snapshot" of the wake, a unique capability that cannot be matched by most available methods. Due to the turbulent nature of the MOD-2 wind turbine wake, this instantaneous velocity profile is very different from a "time-averaged" profile derived from point measurements using individual sensors, such as hotwire probes, kite anemometers, or cup anemometers. In the turbine wake, all the dynamic processes are actually governed by the shape of the instantaneous rather than the time-averaged profile. Such processes include turbulence generation by shear instability and vortex instability, turbulence and vortex interaction, and turbulent entrainment. Similarly, the instantaneous rather than the time-averaged velocity profile is most critical to the dynamic loading of downwind turbines in a wind farm, especially when the maximum loading is considered. To make a fair comparison with results derived from point measurements, it was anticipated during the planning of the field experiments that averaging of these trails would act to smooth out the turbulent fluctuations

within the velocity profile and result in a profile comparable to an "ensembleaveraged" wake profile. In principle, the process of averaging many trails obtained at similar experimental conditions corresponds to an ensemble-averaging approach.

\subsubsection{Rotor Tip Vortices}

Several tracer methods were adopted in an attempt to visualize the formation and evolution of the vortices generated by the rotor blades. The tracers included large smoke generators, flow streamers, and neutrally buoyant balloons (with and/or without smoke tracers attached).

\subsubsection{Large Smoke Generator}

For 1arge smoke generators, we used Superior White Smoke Pots manufactured by Superior Signals. The flammable chemical, which is stored in a canister, can be ignited manually using a pull tab or electrically using a 9-volt battery. The smoke pot generates a total volume of $14,160 \mathrm{~m}^{3}$ of white smoke in about 5 to 7 minutes. To raise the smoke pot into position (as close as $6 \mathrm{~m}$ downwind of the rotor plane), we designed a cable system tethered to the catwalk of the wind turbine at about $45^{\circ}$ to the vertical downwind of the turbine. A schematic 
of the experimental setup is given in Figure 5.6. Two large pulleys were mounted on the catwalk to facilitate sliding of the tethered cable up and down. A small pulley, which was tied to the cable, was used to raise the smoke pot to the designated level via a small cable. The smoke pot was ignited on the ground level and then raised quickly into position. Two persons were tending the tethered cable, one holding the lower loop of the cable and the other positioning the pot by pulling the tethered cable. They were responsible for keeping the cable taut and for maintaining the relative position of the smoke pot with respect to the rotor blades. A third person controlled the small cable to raise and lower the smoke pot in the vertical direction. A fourth person located upwind directed the crews to place the smoke pot in the optimum position for tracking the tip vortices. Two sets of movie and still cameras were used to record the side and front views (from upwind positions) of the formation and evolution of the tip vortices as they were displayed by the smoke tracer. A number of handheld cameras from various positions were used to record perspective and closeup views of the vortex formation. (More details on the setup and procedure for the smoke pot experiments are provided in Appendix B.)

The smoke tracers were very effective in showing the formation and evolution of the tip vortices for a relatively short time after release. In the presence of turbulence, the smoke tracers diffuse rapidly and can no 1onger "paint" a sharp picture of the tip vortices after the smoke has been released for a relatively short time. Our experience shows that the smoke tracers are not discernable from the background haze or clouds at a distance of 3D or more, where $D$ is the diameter of the rotor. Most importantly, the features of utmost interest, which are generally fine structures such as the core of the tip vortices, lose their identity at about $1 D$.

\subsubsection{Flow Streamers}

Another method used flow streamers attached to the tethered line of a JALBERT ${ }^{\otimes}$ parafoil kite. The kite, loaned to Flow by NOAA Pacific Marine Environmental Laboratories, has an area of $2.8 \mathrm{~m}^{2}$. The streamer/kite setup is

\footnotetext{
Model J-30, Kite Factory, 678 West Prospect, Seattle, WA 98119 (Contact: David Checkley).
} 


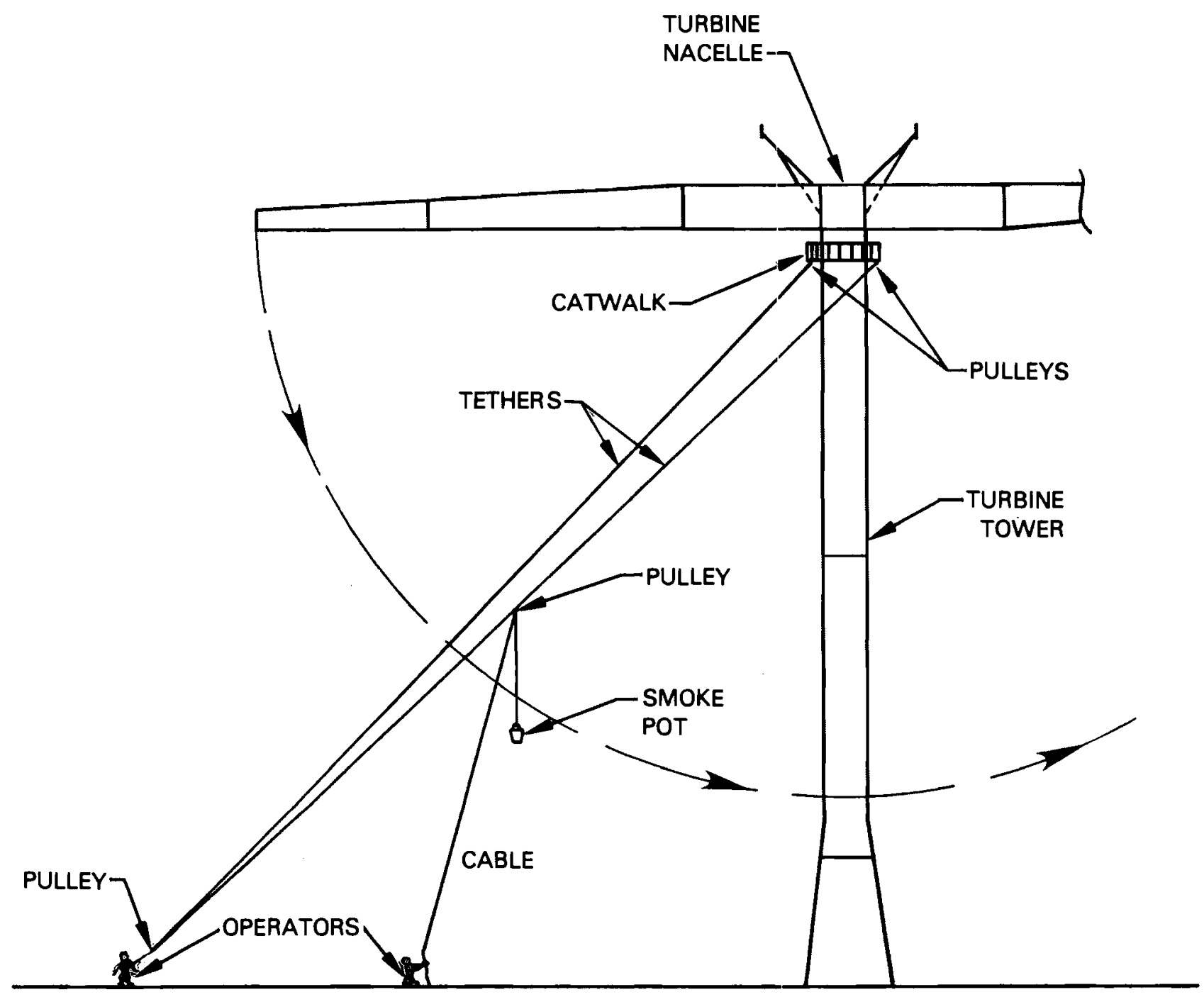

Figure 5.6 Setup of Tethered Cable System for Positioning Smoke Pot (Looking Upwind) 
shown in Figure 5.7. The streamers were made of lightweight material, such as crepe paper or thin fabric strips. The crepe paper worked better than the fabric strips in terms of following the air motion but tended to break off too easily, especially when wet. The streamers were about 2 to $5 \mathrm{~cm}$ wide and 11 to $12 \mathrm{~m}$ long and were attached to the tethered line at about 12-m intervals. The streamers were fluorescent in color and, under the illumination of sunlight, showed good contrast against a blue sky with the use of a polarizer filter. Twelve streamers were attached to the tethered line with half below and half above the edge of the top of the wake. We anticipated that the organized flow pattern, a reminiscence of the tip vortices generated by the rotor blades or large-scale eddies within the wake, would lead to a contrasting motion of the streamers below and above the wake edge. Most of the time, the 1 ine length of the kite was about $200 \mathrm{~m}$. With a $45^{\circ}$ inclination angle, the vertical height of the kite was about $150 \mathrm{~m}$. The top streamer was either at about $140 \mathrm{~m}$ or $130 \mathrm{~m}$ (30 $\mathrm{m}$ from the kite).

\subsubsection{Balloon Tracers}

Three sizes of balloons filled with helium were released upwind from the tethered cable to observe the tip vortices in a Lagrangian frame of reference. only very limited tests were conducted because we found that the results obtained from the balloon tests did not offer additional information to those obtained from the smoke tests using the smoke pot.

The smaller size balloons, with dimensions of 4 to $10 \mathrm{~cm}$ in diameter and of 20 to $40 \mathrm{~cm}$ in length, were purchased at toy shops. The larger ones were $30 \mathrm{mg}$ meteorological balloons with a maximum diameter of about 1 meter. These were used to provide the necessary buoyancy to carry an orange smoke candle ( 4 oz $)$ aloft. When released from upwind of the turbine, the balloons were made slightly buoyant so that they rose into the wake when they reached the turbine.

The balloons released from the tethered cable were neutrally buoyant. Several methods of release were tested. For the smaller balloons, heated nichrome wire loops were used to trigger the release of the balloons. A nylon string was tied to the wire at one end and to the balloons at the other end. To release the balloons, the nichrome wire loops were heated from a 115-volt (ac) power source via an extension cord. A voltage regulator was used to 


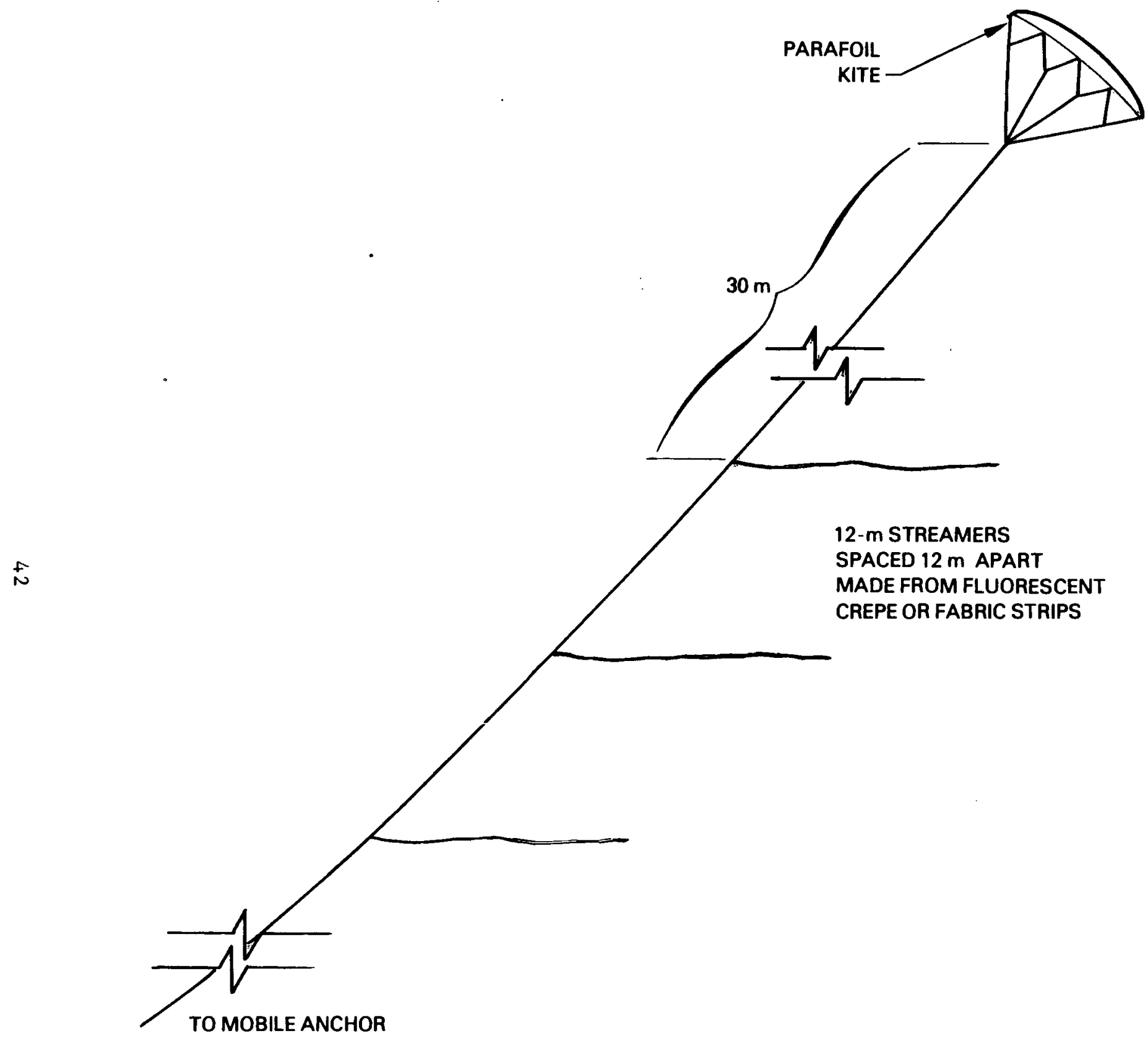

Figure 5.7 JALBERT Parafoil Kite with Flow Streamers 
increase the voltage from zero to the voltage required for melting the nylon strings. For the larger balloons, the tip vortices caused a violent motion of the balloons, which caused the wire loops to break easily. The largest balloons (with the smoke candles) were released upwind of the turbine.

\subsubsection{Wake Height}

Two methods were selected for providing an estimate of the maximum height of the turbine wake as a function of the downwind distance. The two methods made use of the visual results of the rocket smoke trails and of the streamers on the parafoil kite. A smoke trail, once it is laid in the air, is advected with the wind. Above the wake, the smoke trail travels with the ambient wind. Within the wake, the speed of the smoke trail is retarded in the presence of a momentum deficit. As a result, the profile of the smoke trail is distorted. Such a distortion increases with time after the trail is laid (see Figure 5.1). Furthermore, the wake turbulence increases the diffusion rate of the smoke trail within the wake. The portion of the trail within the wake disappears sooner than that above the wake (Figure 5.1). Near the ground, the topographygenerated turbulence is so strong that the smoke trail there is diluted even more quickly than that elsewhere in the wake. The top edge of the wake can be determined from the distorted smoke trail. When the rocket coincides with the wake axis, the maximum wake height can be obtained.

The streamers are capable of following the air motion and are designed to detect the wake-generated organized motion, a reminiscence of the tip vortices and the swirl induced by the rotor motion. Such organized motion is less dissipative than wake-generated turbulence and, consequently, is expected to persist in the far wake. With the streamer section centered about the top edge of the wake, one may in principle estimate the wake height from the vertical positions of the streamers at which their motion shows a distinctive discontinuity. In practice, the streamers have to be designed so that they can respond to the differences in motion to be detected. In this regard, the crepe paper works better than the fabric strips. 


\subsection{PHOTOGRAPHIC EQUIPMENT}

To record the results of flow visualization, we used both still and movie cameras. The still pictures were used for data analysis because the resolution and contrast are superior to those of the movie records. Two of the still cameras were equipped with a motor drive to advance the film at 4 or $2 \mathrm{frames} / \mathrm{sec}$; 50- and 28-mm lenses were used with the two cameras. Several other $35-\mathrm{mm}$ still cameras were used for recording snapshots and closeup pictures of interesting features. Two 16- $\mathrm{mm}$ cameras were used at a film speed of $48 \mathrm{frames} / \mathrm{sec}$ for recording motion pictures. The movie cameras used a 30-m film cartridge. The cameras were equipped with wide angle and zoom lenses and a set of filters. The most frequently used filters were the polarizer filter for color films and the red and yellow filters (with the use of the polarizer) for the black and white films. These filters are capable of enhancing the contrast between the smoke and the blue sky (which is darkened).

Several types of films were used depending on the illumination and sky conditions. For movies, we used either Eastman Kodak Ektachrome films with an ASA of 160 (ASA 400 for poor to fair illumination conditions) or Kodak Tri-X films with an ASA of 400 , depending on the illumination. For still pictures, we used Eastman Kodak Ektachrome films with an ASA of 200 (400 for poor to fair illumination conditions), Kodak Tri-X films with an ASA of 400 , or ALFA films with an ASA of 200.

\subsection{DATA ANALYSIS}

The most quantitative technique pursued during this study was the method of launching model rockets vertically through the MOD-2 turbine wake, monitoring the downwind advection of the smoke by the method of time-lapse photography. Analysis of the photographic sequence can give important information on the structure of the wake. To analyze the photographic record, the photographs were digitized, the data were adjusted to account for errors, and the vertical velocity profiles were plotted. Two quantities were then available from the velocity profile plots: 1) a relative velocity deficit of the wake

\footnotetext{
-Eastman Kodak Company, Rochester, N.Y. 14650
} 
compared to the free-stream velocity and 2) an effective wake height as measured by the portion of the rocket trail having a strong velocity shear or a discontinuity in the diffusion rate of the smoke trail. In the following subsections, the methods of digitization, error estimation, and data analysis are described.

\subsubsection{Rocket Trail Digitization}

The sequence of $35-\mathrm{mm}$ slides showing the advected rocket smoke trails were digitized for later analysis. This was accomplished at Flow by means of a microcomputer-digitizer system consisting of a microcomputer and a digitizer. The slides are projected onto the rear of a $70 \times 70 \mathrm{~cm}$ translucent tablet. The $x$ and $y$ coordinates of the handheld digitizing pen on the activated tablet are transmitted serially to the microcomputer and stored on a floppy disk. Format of the data on the random access file consists of a position available for data point indexing in addition to the paired data points.

A scale factor is entered into the digitizer to produce digitized points having a magnitude corresponding to the measured distance in meters at the launch pad. This scale factor is a quantity that is photographically determined by viewing a pair of flags spaced a known distance apart from the camera positions.

Appendix $C$ details the required structure of the sequential file resulting from the digitizer. Essentially, a series of rocket trail photographs is projected sequentially on the digitizer tablet. Care is taken to overlay the slides such that a shift in the smoke position corresponds to a true advection of the wind, though a correction is made for any offset. The digitizing pen is operated in TRACK mode, in which a stream of points is output to the com puter when the pen is traversed along the rocket smoke trail. File delimiters are placed between each photograph digitization. Once digitized, and stored on the microcomputer, the file of sequential rocket trails is transferred to a mainframe computer, where computations are performed to correct for lens distortion and to produce the velocity profile.

As many as 12 rocket tracks were digitized for any one launch. This was done to examine the evolution of the structure and because by the time the rocket reached its maximum height the lower smoke had diffused. Thus, the 
velocity determination at lower elevations is made with the first few rocket trail digitizations, and the last few tracks are used to determine the velocity profile at the highest elevation.

\subsubsection{Rocket Trail Data Analys is}

Analysis of the smoke trail data to produce a velocity profile of the MOD-2 wind turbine wake principally involves correcting the position coordinates of the digitized rocket trails due to camera lens distortion. This distortion can affect absolutely measured quantities in both the horizontal and vertical dimensions, since the camera views a linear distance having a constant scale on a spherical surface. For the rocket trail data, the lens distortion must be compensated for in the following ways:

1. The horizontal position of the smoke must be adjusted if the instantaneous wind direction does not lie on the chosen launch pad orientation. This correction is determined from the geometry of Figure 5.8. Scaling the output of the digitizer to real physical units at the rocket launch pad location suggests that all measured distances are in scale along the circle of constant scale having radius $R$, as shown in Figure 5.8. Thus the measured distance $S_{x}$, the angle $\theta$, and offaxis camera position $R$ must be used to compute the actual advected smoke trail distance $\chi$. Since for small $B$,

and

$$
\begin{gathered}
X=S_{x} / \cos (\beta+\theta) \\
B=\frac{S_{x}}{R} ;
\end{gathered}
$$

then

$$
X=R B / \cos (B+\theta) \text {. }
$$

In the lens distortion correction procedure, each measured downwind point is corrected with Equation $(5.3)$.

2. A vertical compression of distance that increases with elevation must similarly be corrected to provide reliable estimates of the smoke trail height. The geometrical construction of Figure 5.9 illustrates 


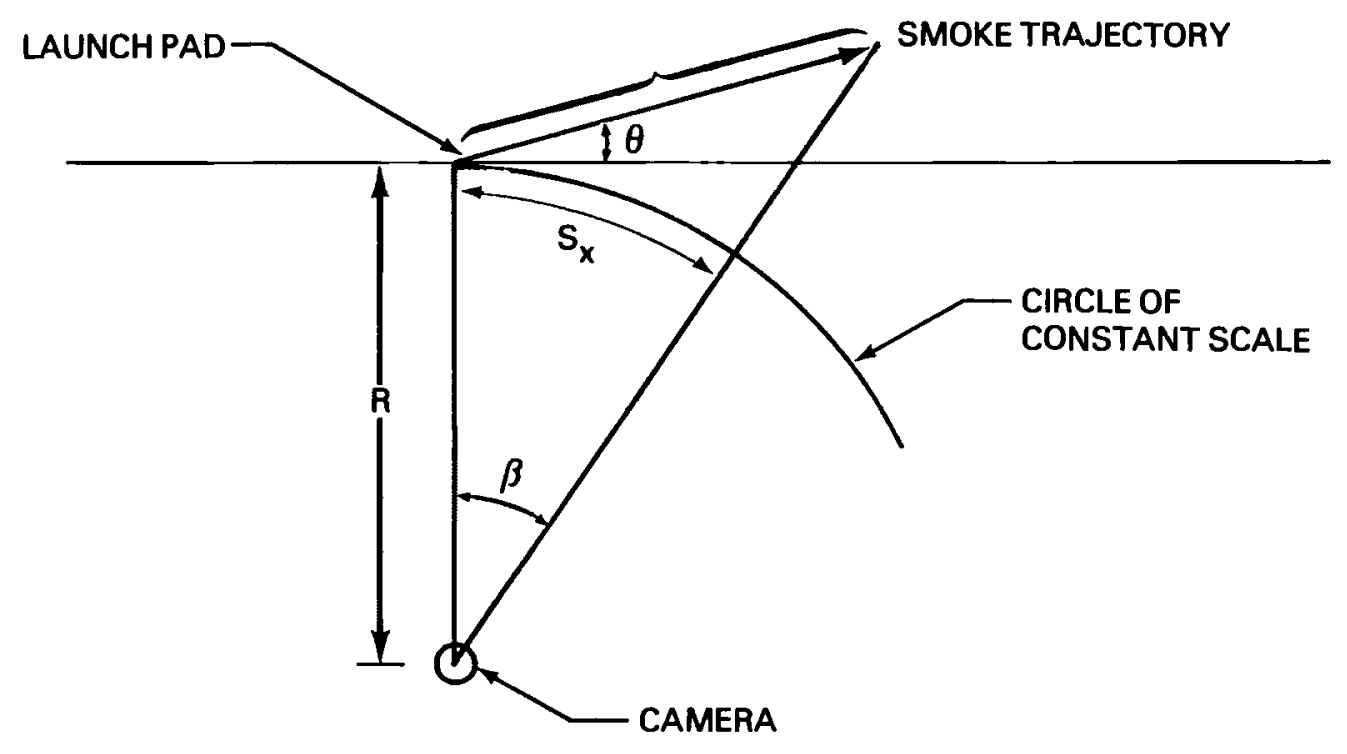

Figure 5.8 Horizontal Correction of Rocket Smoke Trail Position Data 


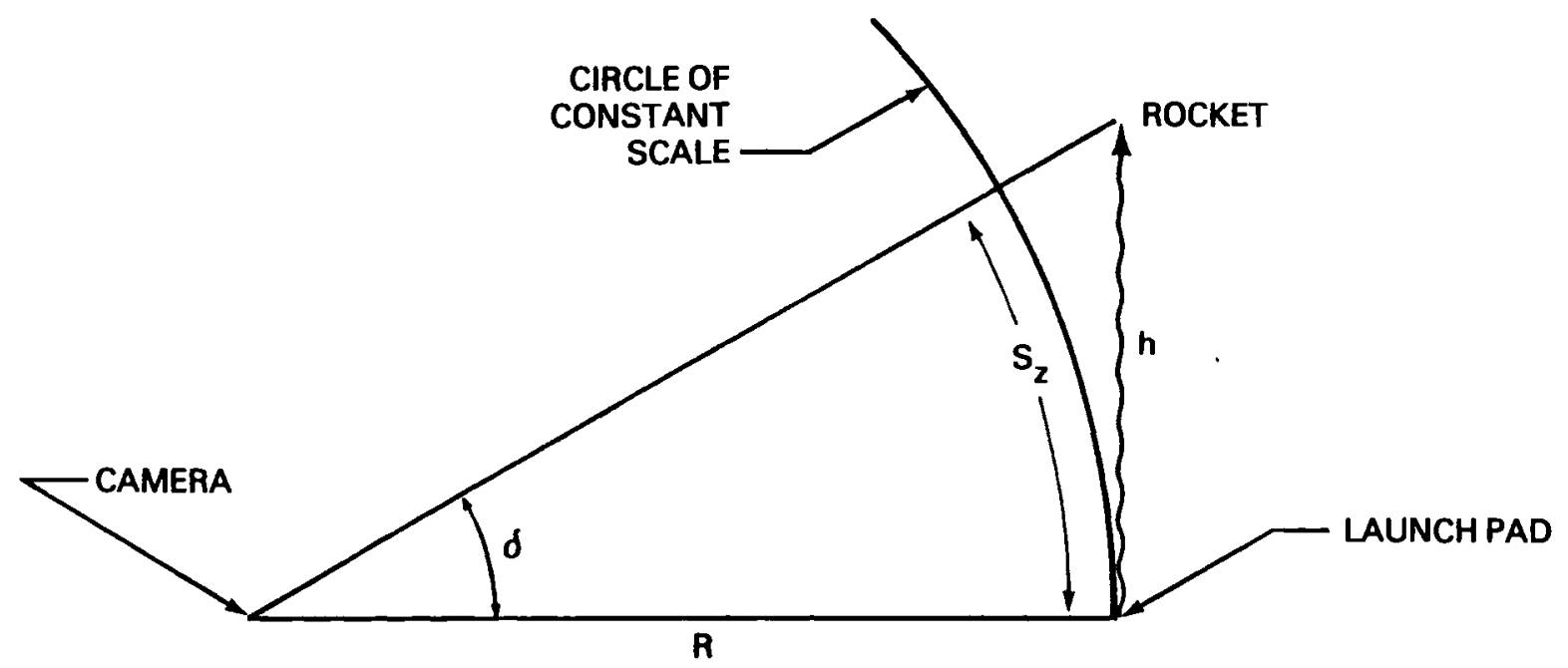

Figure 5.9 Vertical Correction of Rocket Smoke Trail Position Data 
this. Since $\delta=S_{z} / R$, where $S_{z}$ is the quantity as measured by the digitizer,

and

$$
\begin{aligned}
& \tan \delta=\frac{h}{R}, \\
& h=R \tan \left(\frac{S_{z}}{R}\right)
\end{aligned}
$$

then

This equation implies that the vertical correction becomes very large as $S_{z} / R$ approaches $\pi / 2$. During the experiments, both 28 - and 50-mm focal length camera lenses were used from off-axis ( $R$ ) distances of about $140 \mathrm{~m}$. Maximum measured digitizer heights were about $160 \mathrm{~m}$, resulting in a corrected height of $310 \mathrm{~m}$. However, top-ofthe-wake heights were measured at roughly $100 \mathrm{~m}$, and the corrected height therefore was only $120 \mathrm{~m}$. Clearly, errors in measurement are greater for digitizer outputs toward the top of the rocket trail. A check of the vertical elevation correction has been performed by laying out a typical launch pad/camera geometry at the PNL tower. Photographs of the 106.7-m tower were corrected by the same means as were these of the rockets, and the error in correct height determination was $2 \%$ using the 50-mm lens and $4 \%$ using the 28 -mm 1 ens.

3. A third distortion, which becomes larger with elevation, is in the measured distance between successive rocket trails. As viewed in Figure 5.10, this separation becomes small as the rocket approaches a high elevation relative to the camera baseline. A relationship must be formed such that

$$
L=f\left(p, s_{z}\right)
$$

since both $\mathrm{p}$ and $\mathrm{S}_{\mathrm{z}}$ are measured by the digitizer. Now,

$$
\frac{\mathrm{P}}{\mathrm{r}}=\frac{\ell}{\mathrm{R}}
$$

Thus, for sma11 angles,

$$
L \simeq \ell=\frac{\mathrm{pR}}{\mathrm{r}}
$$




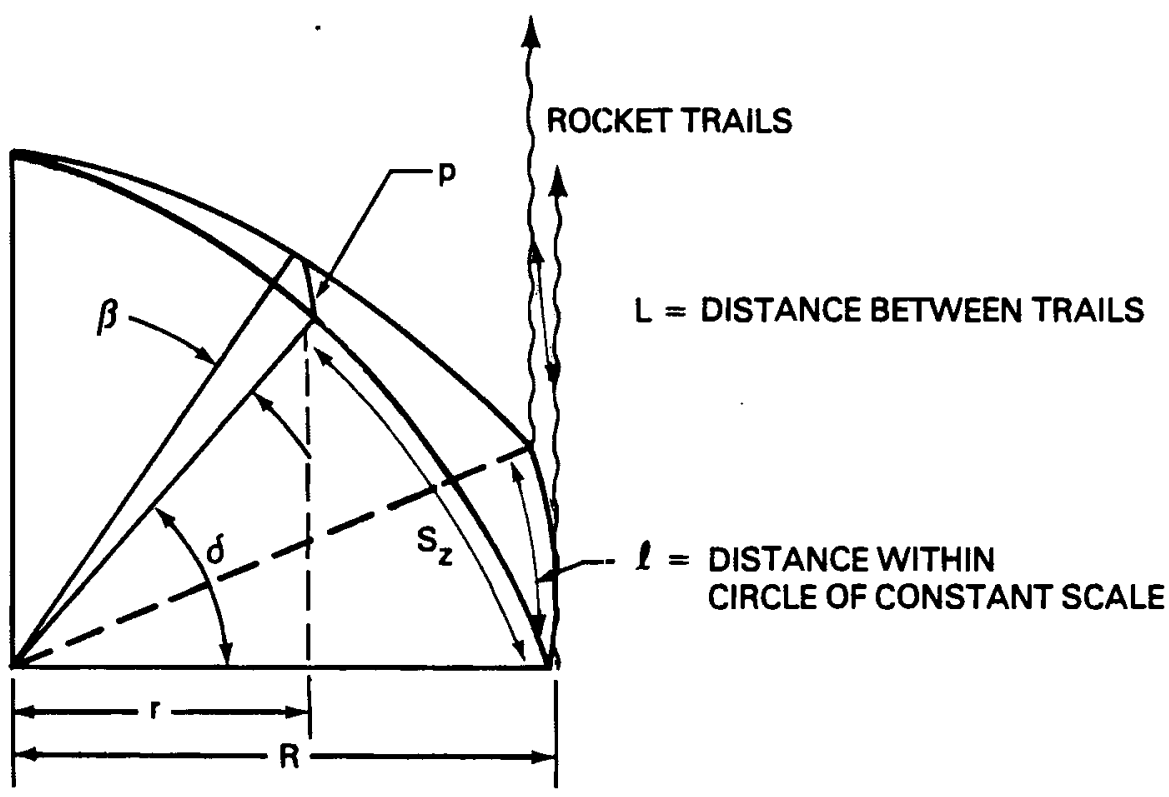

Figure 5.10 Vertical Segment Correction Between Successive Rocket Smoke Trail Position Data 
Since

$$
r=R \cos \delta
$$

then

$L \simeq \frac{p}{\cos \left(S_{z} / R\right)}$

4. As the rocket smoke trail advects downwind, the distance between the camera and the smoke will change, and thus the scaling factor as determined by the launch pad/camera distance ( $R$ ) will not apply without correction. An assumption can be made at this point, however, to avoid this complication. The velocity determination is made by measuring the separation between two successive smoke trails. If it is required that this distance is much less than the launch pad/camera baseline, then the resulting camera distortion will be negligible. For all the velocity profile determinations, the profiles were calculated using smoke trails at a position no farther than $20 \mathrm{~m}$ downwind. For a 180-m baseline, the change in scale over this downwind distance can be considered negligible.

\subsubsection{Tracking the Wake Centerline}

Since only a single rocket is launched within the wake at any one time, an estimation must be made of the launch position with respect to the wake center1 ine so that an overall mapping of the wake structure can be made. This estimation must encompass a possible curvature of the wake path due to terrain features. Analysis of the tower data at the Goodnoe Hills site indicates that a curved wake path may in fact be the norm, as the BPA and PNL meteorological towers consistently show a systematic deviation in wind direction from one another.

With reference to Figure 5.11, the off-axis distance $r$ must be determined as a function of downwind distance and the wind directions at the BPA and PNL towers. The double arrows in the figure represent orientation directions from true north. The direction $\phi$ is the designated turbine axis angle from which the rocket is launched. The wind directions at wind turbine 3 3 and at the BPA and PNL towers are designated $\theta_{0}, \theta_{\mathrm{BPA}}$, and $\theta_{\mathrm{PNL}}$, respectively. The mean wind direction at the launch pad, $\gamma^{-}$, can be deduced from the other wind directions with a few observations and simplifications. For all of the rocket runs in 
question, the wind direction at the BPA tower was between $240^{\circ}$ and $280^{\circ}$. Since the orientation of the PNL tower from turbine 3 is about $260^{\circ}$, and since $\theta_{0} \simeq \theta_{\mathrm{BPA}}$ due to proximity, a linear interpolative procedure may be used to determine wind directions downwind of the turbine and between the two towers. This scheme results in the equation,

$$
\frac{\bar{\gamma}-\theta_{\mathrm{BPA}}}{\theta_{\mathrm{PNL}}-\theta_{\mathrm{BPA}}}=\frac{x}{8.3},
$$

where $\mathrm{x}$ is expressed in rotor diameters. Thus,

$$
\bar{\gamma}=\frac{x}{8.3}\left(\theta_{\mathrm{PNL}}-\theta_{\mathrm{BPA}}\right)+\theta_{\mathrm{BPA}} \text {. }
$$

The off-axis distance $r$ can be expressed by a first-order expression as

$$
r \simeq \mathbf{x}\left(\phi-\theta_{0}+\frac{\varepsilon}{2}\right)=x \theta_{\text {eff }}
$$

where

$$
\varepsilon=\bar{\gamma}-\theta_{0} \simeq \bar{\gamma}-\theta_{\mathrm{BPA}}
$$

The term $\varepsilon$ represents a correction due to a transverse advection of the wake downstream. Combining Equations (5.12) and (5.13) gives

$$
\theta_{\text {eff }}=\left(\theta_{\mathrm{BPA}}-\phi\right)+\frac{\mathrm{x}}{16.6}\left(\theta_{\mathrm{PNL}}-\theta_{\mathrm{BPA}}\right) \text {, }
$$

and a simple expression for calculating the off-axis distance $r$ has been determined, since $r=x \theta_{\text {eff }}{ }^{\circ}$ By convention, this expression holds when the effective wake centerline is north of the rocket launch baseline. For the opposite case, we take $\theta_{\text {eff }}$ to be negative.

\subsubsection{Computer Program}

A computer program named DEFICIT was developed to read the digitizer data files, correct the data for lens distortion, create a plottable data file of the corrected position data, and calculate the velocity profile. A description of the program, the program listing, and sample printouts are included as Appendix $C$. 


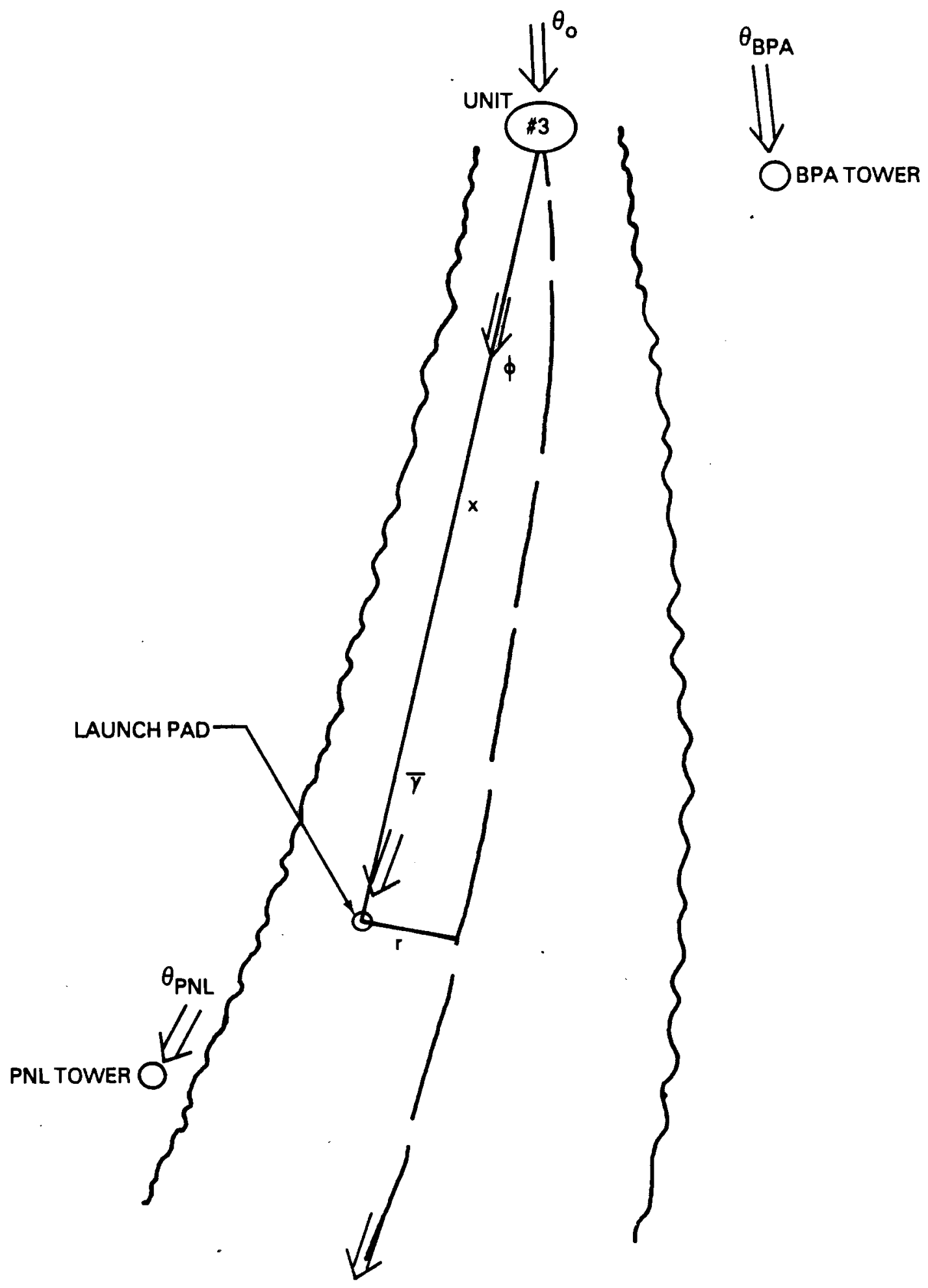

Figure 5.11 Geometry of the Curved Wake of Turbine \#3 •

The launch pad is shown a distance $r$ from the wake centerline. Double arrows indicate the orientation direction from true north. 


\subsubsection{Estimated Errors in the Rocket Trail Data}

A number of factors can critically affect the results of the rocket trail experiments. For valid conclusions to be made based on the smoke trail measurements of wake height and velocity deficit, an accurate placement of the smoke trail within the wake is crucial. The analysis above provides a determination of the off-axis distance $r$, but this is a time-averaged quantity. For example, to obtain a spatial average of the wake position, the linear interpolation scheme as outlined in Section 5.3.3 requires time-averaged quantities of $\theta_{\mathrm{BPA}}$ and $\theta_{\mathrm{PNL}}$. A simple 2-min average of these quantities was chosen on the basis that the "time constant" of the wake position is of this order. This "time constant" can be thought of as having a magnitude close to the advection time of an air parcel passing from turbine $\$ 3$ to the rocket launch pad.

A number of errors can result from this procedure. In the near wake, the 2 -min average is not very representative due to the short downwind advection time of the air parcel. In the far wake, terrain features could cause the wake to meander independently of upstream wind directions. Thus, a possibly large and unquantifiable error can enter into the calculations of the off-axis distance $r$.

In determining the effect of this error, it is important to re-examine the goals of this visualization experiment. From the rocket trail data, a goal was set to obtain velocity deficit and wake height information. A statistical approach was pursued based on the above random errors. All rocket runs made under similar meteorological conditions and at the same downwind distance were plotted together on a graph having the off-axis distance $r$ as the abscissa. The ordinate was either the velocity deficit or the wake height elevation. When enough runs were plotted in this way, a statistical confidence in the trend of the data resulted. Thus, we were able to overcome the large ambiguity in the wake centerline determination.

The change in wind direction with height is not considered to be important in the vertical region of the wake. In the -near wake, close to the roll-off in topography on the west side of the site, this effect is strongest but is thought to be of lesser magnitude than the large-scale wake meandering due to changes in the vertically integrated wind direction from west to east across the site. 
Another systematic error results in an ambiguity in the vertical placement of the smoke trail position data with respect to the turbine disk. The vertical variation in topography over the area downwind (east) of turbine 非 3 is relatively large. The base of turbine 3 is $5 \mathrm{~m}, 9 \mathrm{~m}$, and $16 \mathrm{~m}$ below the $1 \mathrm{D}$, 2D, and 6D positions, respectively. Since a streamline compression can occur in the flow over this type of terrain, depending on stability conditions, correction of the absolute elevation of the rocket trail for comparison to existing models is difficult. In the interest of consistency, no change in the elevation scale is made to the rocket data. A similar error in height positioning occurs when the camera cannot view the launch pad due to some topographical obstruction. The elevation that is hidden is simply added to the vertical positions as measured by the rocket.

Two relatively insignificant random errors are mentioned for completeness. The method of determining the velocity profile by subtraction of the horizontal positions does not take into account vertical motion in the wake, which obviously exists. The turbulent eddies responsible for this vertical motion do not approach the strength of the downwind advection behind the turbine; so for the most part this is a small error. However, occasionally a "kink" will develop in the velocity profile which then can either move upward or downward. This cannot be recognized by the computer program as being a true vertical motion, so errors result in the position differences between two trails having "kinks" displaced vertically from each other. At worst, this problem will show up in the velocity profile as a small spike having an obvious nonphysical character. Generally, the digitizer operator can recognize this potential problem and avoid digitization of small-scale turbulent "kinks" in the rocket trail photograph.

The second random error can come from the speed of the 35-mm motor-drive camera. The time between frames is set at a nominal $1 / 4$ second, but it has been found that an error as high as $10 \%$ may exist in the timing, depending on the state of the batteries. Before and after each field test period, the timing rate is checked using the trigger input on a digital oscilloscope. In this way an accuracy of better than $3 \%$ is obtained in the timing. 


\section{EXPERIMENTAL RESULTS}

In this section, the results of the visualization experiments are presented and discussed. First, the visual results of the rocket smoke trail experiments are presented, followed by an analysis of the rocket smoke trail data. Then, we present the analysis of the wake height and velocity deficit information derived from the digitized visual data and resultant velocity profile plots. Next, the wake height and velocity deficit data are compared to those from a theoretical model. Finally, the results of the rotor tip vortices visualizations, using smoke tracers, rocket smoke trails, and flow streamers, are presented and discussed. A $16-\mathrm{mm}$ movie film, entitled "Flow Visualization in the Wake of a MOD-2 Wind Turbine" (Liu et al. 1982), summarizes the visual results. Frequent reference to this $\mathrm{film}$ is made to help interpret the results.

\subsection{VISUAL RESULTS FROM SMOKE TRAILS}

In this section, we present typical visual results of the rocket smoke trail experiments, and point out the essential features of the wake that can be extracted from the results. The discussion also lays the necessary ground work for the derivation of qualitative and quantitative results deduced from the smoke trail data.

Figure 6.1 shows seven sequential pictures of a smoke trail released at $1 / 2 \mathrm{D}$ and $275^{\circ}$ for rocket run RT70. The 10-min averaged wind speed and direction, as measured from the BPA tower at $59.4 \mathrm{~m},\left(\right.$ a) are $9.1 \mathrm{~m} / \mathrm{s}$ and $266^{\circ}$. The experimental conditions for the rocket trail experiments are summarized in Table 6.1. The time lapse between pictures is 1 second, with time increasing from left to right. The arrow in the figure indicates the wind direction. Initially, the rocket followed a reasonably straight and vertical course. The Formation of a velocity deficit in the lower portion of the trail becomes evident as time increases. The vertical profile of the velocity deficit appears to be disk-1ike at this station. The presence of a turbulent boundary layer just above the ground is noticeable, as indicated by the relatively rapid. diffusion of the smoke trail. Near the top of the wake, where the velocity

(a) The 10-min averaged wind speed and direction referred to hereafter were measured at the 59.4-m BPA tower. 
Table 6.1. Summary of Rocket Smoke Trail Run Conditions and observations

\begin{tabular}{|c|c|c|c|c|c|c|c|c|c|c|c|c|c|c|}
\hline Run & $\begin{array}{l}\text { Downwind } \\
\text { Distance } \\
(94 \mathrm{~m} / \mathrm{D})\end{array}$ & $\begin{array}{c}\text { Date } \\
(1982)\end{array}$ & $\begin{array}{l}\text { Time } \\
\text { (PST) }\end{array}$ & $\begin{array}{c}\text { BPA } \\
59.4 \mathrm{~m} \\
W . S \\
(\mathrm{~m} / \mathrm{s}) \\
\end{array}$ & $\begin{array}{c}\text { Turbine } \\
\text { Axis Angle } \\
\left(\theta_{\text {eff }}\right)(\text { deg })\end{array}$ & $\begin{array}{c}\Delta \mathrm{T} \\
96.6 \mathrm{~m} \\
\left({ }^{\circ} \mathrm{C}\right) \\
\end{array}$ & $\begin{array}{c}\sigma_{\theta} \\
\text { W.D. } \\
\text { std.dev. } \\
\text { (deg) }\end{array}$ & $\begin{array}{c}\alpha \\
\text { Transverse } \\
\text { Turb. Int. } \\
(\%) \\
\end{array}$ & $\begin{array}{c}r \\
\text { off- } \\
\text { Axis } \\
\text { Position } \\
(\mathrm{m})\end{array}$ & $\begin{array}{c}\text { Measured } \\
\text { Free- } \\
\text { Stream } \\
\text { W.S. } \\
(\mathrm{m} / \mathrm{s}) \\
\end{array}$ & $\begin{array}{c}\quad \text { Mean } \\
\text { Internal } \\
\text { Velocity } \\
\text { Deficit } \\
(\%) \\
\end{array}$ & $\begin{array}{l}\text { Top } \\
\text { Wake Edge } \\
\text { Vert ical } \\
\text { Shear } \\
\left(\mathrm{ms}^{-1} / \mathrm{m}\right)\end{array}$ & $\begin{array}{l}\text { Upper } \\
\text { Wake } \\
\text { Edge } \\
\text { Height } \\
(\mathrm{m}) \\
\end{array}$ & $\begin{array}{l}\text { Lower } \\
\text { Wake } \\
\text { Edge } \\
\text { Height } \\
\text { (m) } \\
\end{array}$ \\
\hline$* \mathrm{RT} 70$ & $\frac{1}{2} \mathrm{D}$ & $9 / 8$ & $16: 12: 03$ & 9.1 & -9.6 & -1.28 & 6.4 & 11.2 & -8 & 9.2 & 42 & 0.16 & 104 & 8 \\
\hline RT 18 & & $8 / 3$ & $18: 23: 04$ & 6.4 & -13.2 & -0.98 & 2.1 & 3.7 & -21 & 5.3 & 49 & 0.51 & 97 & 23 \\
\hline RT28 & & $8 / 4$ & $18: 03: 10$ & 6.8 & +1.8 & -1.06 & 4.7 & 8.2 & +9 & 6.4 & 65 & 0.23 & 130 & 53 \\
\hline *RT30 & 1D & $8 / 4$ & $18: 20: 10$ & 8.2 & +7.7 & -1.08 & 6.0 & 10.5 & +12 & 6.9 & 47 & 1.06 & 94 & 22 \\
\hline *RT67 & & $9 / 8$ & $15: 40: 00$ & 9.1 & -3.5 & -1.62 & 7.0 & 12.3 & -6 & 9.6 & 51 & 0.10 & 132 & 18 \\
\hline$* \mathrm{RT} 68$ & & $9 / 8$ & $15: 46: 27$ & 8.2 & -2.5 & -1.62 & 8.0 & 14.0 & -4 & 8.4 & 50 & 0.33 & 117 & 18 \\
\hline RT42 & & $8 / 30$ & $07: 44: 00$ & 7.3 & +1.0 & -1.19 & 5.2 & 9.1 & +3 & 8.0 & 42 & 0.10 & 92 & 11 \\
\hline RT46 & & $8 / 30$ & $08: 46: 00$ & 7.7 & +15.7 & -1.14 & 5.2 & 9.1 & +50 & 6.9 & 61 & 0.50 & 87 & 36 \\
\hline$* \mathrm{RT} 47$ & 2D & $8 / 30$ & $09: 08: 30$ & 8.6 & -8.3 & -1.37 & 6.4 & 11.2 & -26 & 9.6 & 10 & 0.96 & 45 & 23 \\
\hline$\because$ RT 48 & & $8 / 30$ & $11: 07: 00$ & 7.7 & -6.8 & -1.27 & 11.0 & 19.4 & -22 & 8.7 & 26 & 1.10 & 112 & 22 \\
\hline$* \mathrm{RT} 49$ & & $8 / 30$ & $11: 10: 30$ & 8.6 & -5.6 & -1.30 & 8.1 & 14.2 & -18 & 13.3 & 20 & 0.08 & 157 & 22 \\
\hline$*$ RT51 & & $9 / 8$ & $10: 04: 24$ & 8.2 & -3.6 & -1.63 & 7.8 & 13.7 & -29 & 8.2 & 46 & 0.08 & 120 & 35 \\
\hline$*$ RT5 52 & & $9 / 8$ & $10: 10: 24$ & 6.8 & -3.2 & -1.79 & 9.4 & 16.6 & -21 & 6.7 & 18 & $\sim 0.05$ & 163 & 38 \\
\hline * RT53 & & $9 / 8$ & $10: 32: 20$ & 9.1 & -6.4 & -1.66 & 6.5 & 11.4 & -41 & 6.1 & 51 & 0.09 & 125 & 45 \\
\hline *RT54 & & $9 / 8$ & $10: 36: 26$ & 6.8 & 0.0 & -1.80 & 6.3 & 11.1 & 0 & 6.8 & 0 & - & - & - \\
\hline$\therefore R T 55$ & 4D & $9 / 8$ & $10: 46: 50$ & 7.7 & 0.0 & -1.81 & 9.1 & 16.2 & 0 & 6.6 & 20 & 0.12 & 118 & 55 \\
\hline *RT56 & 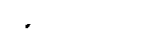 & $9 / 8$ & $10: 56: 05$ & 8.2 & -17.6 & -1.72 & 10.3 & 18.2 & -112 & 9.2 & 26 & 0.10 & 115 & 12 \\
\hline$* \mathrm{RT} 57$ & & $9 / 8$ & $12: 24: 30$ & 8.2 & +6.4 & -1.68 & 7.2 & 12.6 & +41 & 9.2 & 28 & 0.11 & 116 & 32 \\
\hline *RT58 & & $9 / 8$ & $12: 29: 40$ & 9.1 & -10.6 & -1.92 & 7.2 & 12.6 & -68 & 8.4 & $\sim 0$ & - & - & - \\
\hline *RT59 & & $9 / 8$ & $12: 42: 00$ & 8.6 & +7.5 & -2.16 & 6.4 & 11.2 & +48 & 8.7 & 19 & 0.11 & 114 & 24 \\
\hline$* \mathrm{RT} 60$ & & $9 / 8$ & $13: 10: 45$ & 10.0 & -2.3 & -1.90 & 8.4 & 14.7 & -22 & 7.6 & $\sim 0$ & - & 171 & 12 \\
\hline *RT61 & & $9 / 8$ & $13: 48: 20$ & 7.7 & -9.2 & -1.67 & 8.6 & 15.2 & -88 & 9.5 & $\sim 0$ & - & 125 & - \\
\hline${ }^{*} \mathrm{RT} 62$ & & $9 / 8$ & $13: 52: 40$ & 9.1 & +2.2 & -2.03 & 9.6 & 17.0 & +21 & 8.0 & 9 & $\sim 0.015$ & 157 & - \\
\hline RT64 & $6 \mathrm{D}$ & $9 / 8$ & $14: 06: 00$ & 9.5 & -7.2 & -1.49 & 2.5 & 4.4 & -69 & 6.4 & 20 & 0.11 & 127 & 10 \\
\hline$* \mathrm{RT} 65$ & & $9 / 8$ & $14: 13: 20$ & 8.2 & -8.5 & -1.79 & 8.4 & 14.8 & -81 & 7.5 & 15 & 0.11 & 166 & 30 \\
\hline$*$ RT66 & & $9 / 8$ & $14: 18: 20$ & 8.6 & -5.0 & -1.79 & 8.5 & 14.9 & -48 & 7.7 & 13 & 0.16 & 150 & 30 \\
\hline
\end{tabular}

*Runs used for comparison to Lissaman model. 
deficit decreases with height, two cusps form in the very beginning and become fully developed after 3 or 4 seconds. The top cusp develops into a vortex formation which is generated by the rotor tip of the MOD-2 turbine (see discussions in Section 6.6), whereas the lower cusp belongs to the strong shear region of the velocity profile. Note that the integrity of the trail persists for at least 15 seconds (last picture taken), except in the lower $40 \mathrm{~m}$ where terrain-generated turbulence prevails. Even the highly swirling vortex flow surrounding the core of the tip vortex does not appear to be turbulent except maybe in the thin core. The persistence of the integrity of the smoke trail indicates that 1 ittle turbulence is created, in addition to the ambient turbulence already present in the approaching flow, as the wind passes through the rotating blades. This is a very important finding and we will discuss it more in detail after examining more smoke trails at downind stations.

It is interesting to note that the $1 / 2 \mathrm{D}$ profile exhibits no periodicity due to the passage of the MOD-2 blades. The profile instead lends support to the potential core model of the initial momentum deficit, which assumes that the initial state of the wind turbine wake resembles the coflowing jet model discussed in section 3. The observations support this thesis, though a physical interpretation is in order. It is not expected that the upstream wind speed, upon encountering the MOD-2, experiences a discontinuous change in magnitude. Instead, the upstream blockage by the turbine may cause a gradual decrease in wind speed (a momentum deficit) as the turbine is approached so that there is very little change in speed as the flow passes through the turbine disk. The relatively low solidity of the rotating blades, as compared to an obstacle, reduces the magnitude of the momentum deficit and the disturbance to the flow passing through the turbine.

Figure 6.2 is another sequence of pictures of two smoke trails, one in the wake (left) and the other ( $r i g h t$ ) away from the wake, launched simultaneously at 1D. The "in-wake" rocket was launched at $275^{\circ}$ and the "reference" rocket was launched at about $1 \mathrm{D}$ behind the in-wake one. The time lapse between pictures is also $1 \mathrm{~s}$ in this case. The wind speed and direction are $8.2 \mathrm{~m} / \mathrm{s}$ and $283^{\circ}$, respectively. The relatively poor contrast in the lower portion of the picture is caused by the presence of haze in the horizon. From the in-wake trail, we may define easily the wake region in which a velocity deficit 


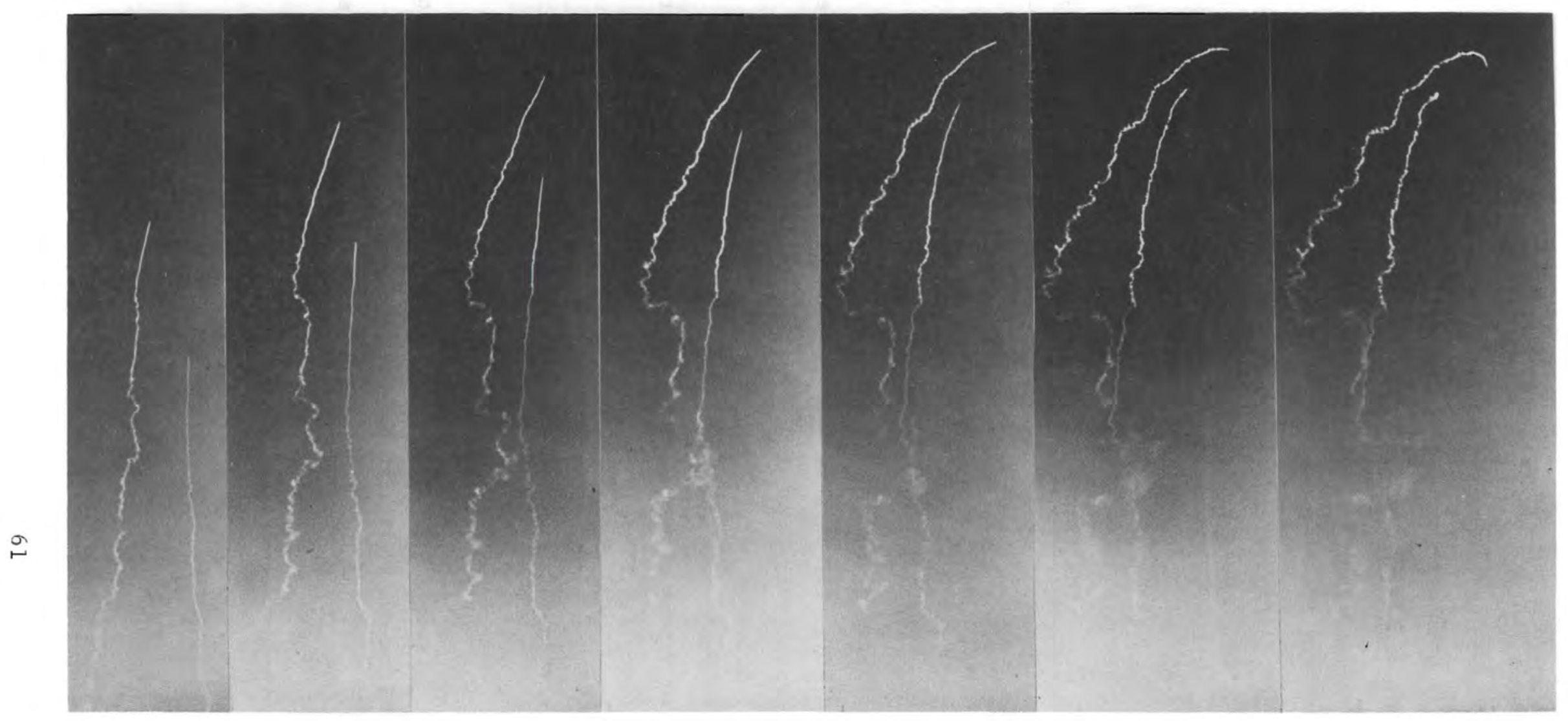

WIND DIRECTION

\section{Figure 6.2 Sequential Pictures of Two Smoke Trails [RT30 and RT31].}

The in-wake trail (left) is at $1 \mathrm{D}$ and $\phi=275^{\circ}$ and the reference trail (right) is about $1 \mathrm{D}$ behind the in-wake trail. The pictures were taken looking south, and the time between two pictures is 1 second.

Comparison of the two trails clearly demonstrates the presence of a velocity deficit in the turbine wake. 
develops (the criterion based on the velocity deficit). The velocity deficit becomes very distinctive and even more so when we compare the time evolution of the relative displacement of the two trails. The velocity deficit appears to also be disk-1ike at ID, as estimated from the shape of the smoke trail. A much larger deficit was observed in the middle portion of the wake and is be1 ieved to be the nacelle wake although we do not see it again from all other profiles including that at $1 / 2 \mathrm{D}$. Above the wake region where a deficit exists, the integrity of the reference trail deteriorates very slowly, indicating very low level ambient turbulence. Inside the wake region, the integrity of the trail deteriorates somewhat sooner. From the difference in the turbulence level inside and out of the wake (the criterion based on the turbulence level), we may also determine the wake height to be the vertical distance at which the diffusion rate of the smoke shows a discontinuity. From the figure, we find that the wake heights estimated from the two criteria, one based on the velocity deficit and the other on the turbulence level, are consistent and differ only slightly. From the original slides of the pictures, the entire trail is still discernable after $7 \mathrm{~s}$ (the last picture shown), indicating the turbulence level in the wake is still relatively low. It is evident that the lower portion of the reference trail diffuses more slowly than does the same portion of . the in-wake trail, as anticipated. Based on the above discussion, we feel confident to define the wake height as the height at which the velocity deficit vanishes, as long as a velocity deficit can be identified. Note that the wake height so defined is not necessarily the maximum wake height because the trail may not coincide with the turbine wake axis. More discussion on this subject and a method to estimate the off-axis distance are presented in section 6.3. Gareful examination of the reference trail shows a slight velocity excess in the same region where a deficit is observed from the in-wake trail. Such an excess can be seen clearly from enlarged photographs of the first, third and fifth pictures in Figure 6.2, as shown in Figure 6.3. Apparently, the presence of the wind turbine creates a partial blockage to the approaching flow that causes the outside flow to speed up slightly. The degree of speeding up is expected to be small due to the low solidity of the wind turbine as an obstacle to the flow. 

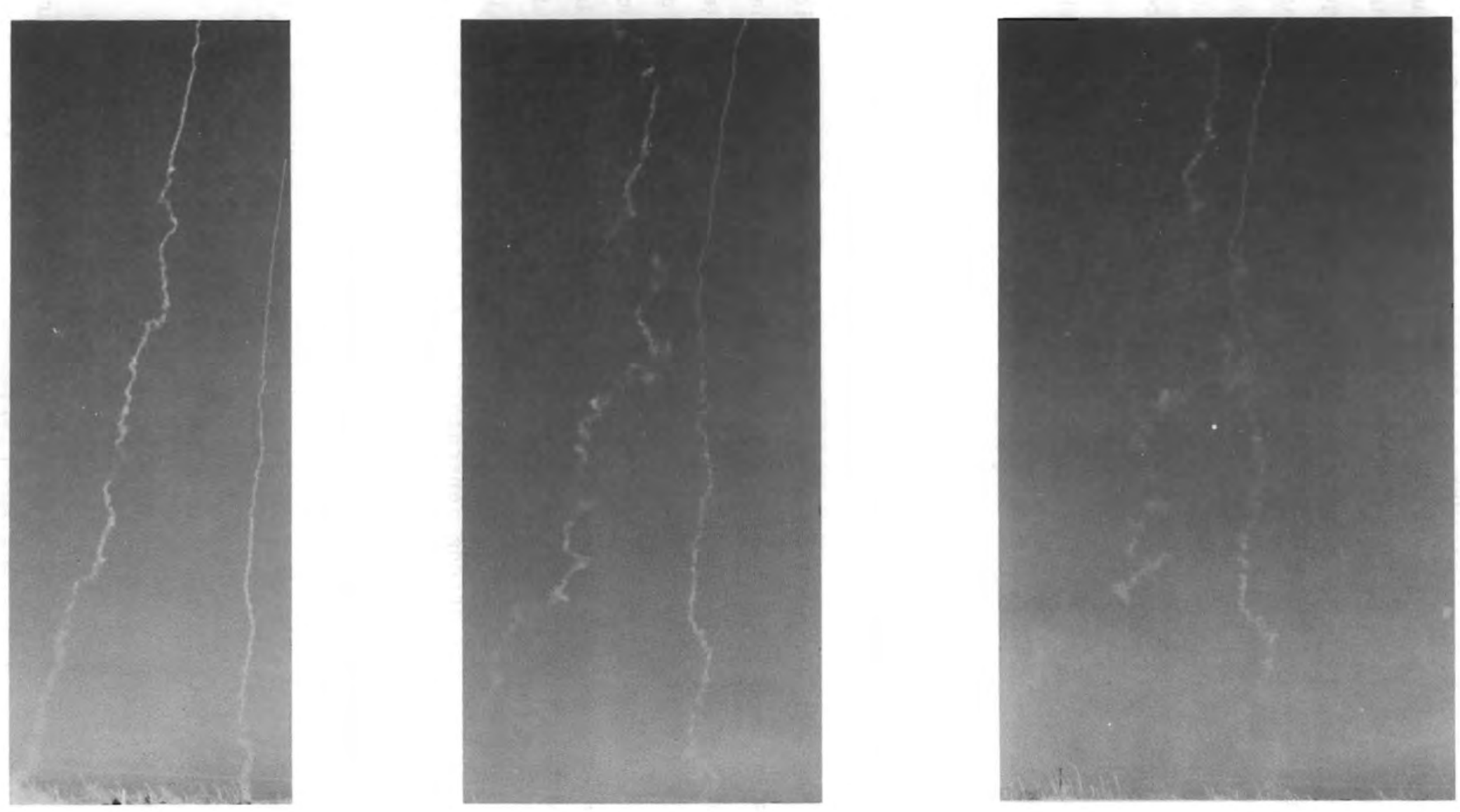

WIND DIRECTION

Figure 6.3 Enlarged Photographs of Smoke Trails Selected from Figure 6.2 [RT30 and RT31] •

Note the difference in the integrity of the in-wake and reference trails as an indicator of the turbulence level. 
Figure 6.4 shows yet another sequence of pictures of a smoke trail released at $6 \mathrm{D}$ and $255^{\circ}$ for run RT66. The wind speed and direction are $8.6 \mathrm{~m} / \mathrm{s}$ and $250^{\circ}$. The velocity deficit is still observable but is not as clear as that in Figures 6.1 through 6.3. However, the wake region can be clearly distinquished from the ambient region above the wake by the appearance of the trail, that is, the difference in the integrity of the trail. In other words, we ray adopt the criterion based on the turbulence level, as discussed above. Tn the far waice where the velocity deficit is difficult to observe, we will use this criterion to determine the wake height. In the near wake, however, we may use the above criterion or that based on the velocity deficit as described earlier in this section.

Qualitatively, the time in which the in-waice trails maintain their integrity tends to decrease with increasing downwind distance. At $1 / 2 \mathrm{D}$, we find very low level turbulence in the wake, which implies that very little turbulence is created as the flow passes the rotating blades. Momentum is being extracted from the approaching flow to drive the turbine blades. A portion of the extracted momentum is deposited back in the wake via tip vortices generation. The swirling flow surrounding the core is coherent but not very turbulent. At downwind stations, the turbulence level tends to increase due to wake instability, such as shear and vortex instabilities, vortex-turbulence interaction, and other potential sources. This finding is not entirely consisrent with the results derived from wind tunnel simulation using a porous disk, a tea strainer, or a scaled model rotating at a relatively high speed. All these turbine "simulators" are strong generators of turbulence and momentum deficit. Therefore, in the very near wake, the turbulence level and velocity deficit in a simulated wake are expected to be higher than those in a fullscale wake. Thus, the validity of the simulated results should be examined with discretion, especially as they have been applied extensively to verify various numerical inodels of wind turbine wakes.

\subsection{ROCKET SMOKE TRAIL DATA}

Table 6.1 shows a sumnary of all successful rocket lannches performed during the months of August and September. A fey preliminary launches were made during July as part of the technique testing program, but all of these 

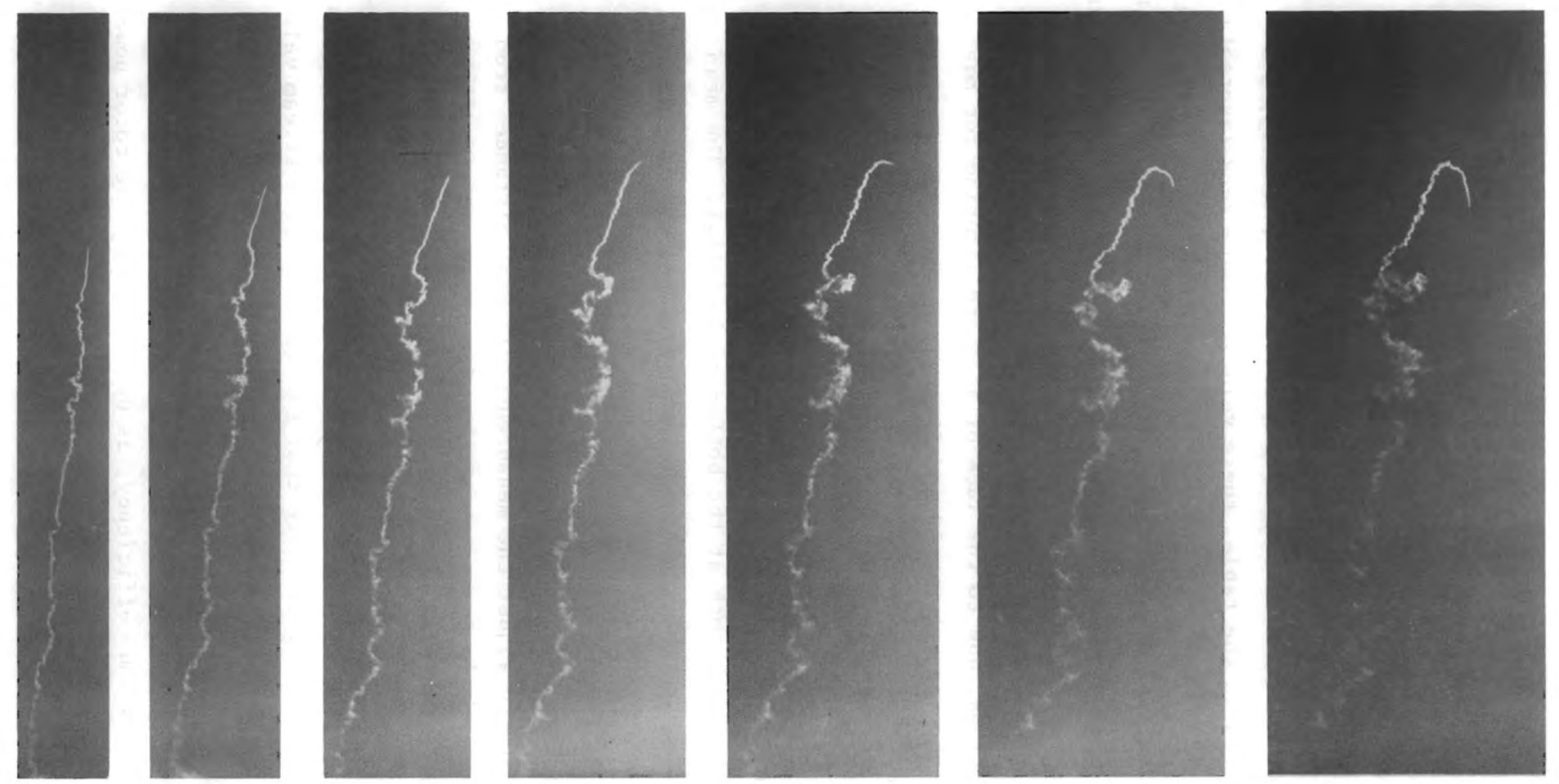

WIND DIRECTION

Figure 6.4 Sequential Pictures of a Single Smoke Trail at 6D and $\phi=255^{\circ}$ [RT66] .

The pictures were taken looking south, and the time between two trails is 1 second. Note the difference in the integrity of the smoke traces above and below the top edge of the wake. 
rocket trails were photographed with a 50-rom lens, later proven to have too sinall an angle of acceptance for wake height measurements. All of the 4D and 6D runs took place on September 8. This day dawned clear and warm and hail very steady wind speeds, as shown by the BPA $59.4-\mathrm{m}$ wind speed column in the table. The criteria for comparison of rocket smoke trail results, as outlined above, include similar conditions of wind speec, turbulence intensity, and stability. As can be seen in the table, these values compare very favorably Eor two of the 1D runs [RT67 and RT68], three of the 2D runs [RT47, RT48, and . RT49], all of the 4D runs, and all but one of the 6D runs [RT64], all of which were performed under highly unstable conditions. In addition, the single $1 / 21$ run has turbine and meteorological conditions similar to these particular rocket launches. None of the smoke trail experiments were performed under stable night-time conditions, due to the lack of adequate 1 ighting for tine photographs. A few of the runs at the 10 and 20 positions were only maryinally unstable. This is a shortfall in the completeness of the data base that had been set as a goal prior to the onset of field work. Though each individual rocket experiment can show important features of the wind turbine wake, only the highly unstable runs are compared to each other. The runs ased in the comparison are indicated with a note at the botcom of Table 6,1. The mean results of this comparison are then related to a theoretical model.

Several observations are evident from Table 6.1. Free-stream wind speeds as measured at the BPA $59.4-\mathrm{m}$ level are $8.2 \pm 1.8 \mathrm{~m} / \mathrm{s}$ for all the compared rocket smoke trail runs. Using this information, a check can be made of the computer program's ability to adjust the measured horizontal distances fron the digitizer due to an off-axis component of the wind. Most of the freestream wind speeds as measured from the plotted velocity profiles at $x \leq 1 D$ compare very favorably (within approximately 15\%) with the BPA tower wind speeds. The agreement deteriorates with increasing distance between the tower and the rocket station, as expected. Note that the measured free-stream velocity is generally at a level higher than $59.4 \mathrm{~m}$, thus some deviation between the two measurements is expected.

The occurrence of wind speeds averaging about $8 \mathrm{~m} / \mathrm{s}$ implies that the turbine is operating at near maximum efficiency, as determined by the rotor power 
coefficient, $\mathrm{C}_{\mathrm{p}} \cdot{ }^{(a)}$ The relatively flat portion of the $\mathrm{C}_{\mathrm{p}}$ curve 1 ies between 7.5 and $9.5 \mathrm{~m} / \mathrm{s}$. Thus, the velocity deficits as measured from the profiles reflect the maximum amount of upstream turbine effect, which facilitates comparison of the results with presently available wind turbine wake models.

The measured transverse turbulence intensities in Table 6.1 all show that rather similar turbulent conditions existed for the highly unstable runs. The median of all the values is about $14 \%$, as determined by the approximate equation

$$
\alpha=\frac{\sigma_{\mathrm{v}}}{\overline{\mathrm{U}}} \simeq \frac{\overline{\mathrm{U}}_{\mathrm{BPA}} \tan \sigma_{\theta}}{\overline{\mathrm{U}}_{\mathrm{BPA}}} \simeq \tan \sigma_{\theta}
$$

where $\alpha$ is the transverse turbulence intensity, $\bar{U}_{B P A}$ the 59.4-m BPA tower wind speed (2-min average), and $V \simeq \sigma_{v}$ the transverse wind speed. The approximation for $\sigma_{v}$ in Equation (6.1) has been shown by Lumley and Panofsky (1964) to be close in magnitude to the exact expression.

Also in Table 6.1, a column entitled turbine axis angle $\left(\theta_{\text {eff }}\right.$ ) gives the effective angle between the wake centerline and the rocket launch pad, as measured from the base of turbine $\equiv_{3}$. As developed in section 5.3.3, this angle can be determined from Equation (5.15), which computes the migration of the wake downwind based on the BPA and PNL 59.4-m wind directions. The offaxis position $r$ (Table 6.1) can then be computed from the angle $\theta_{\text {eff }}$ using Equation (5.13). Negative signs on both $\theta_{\text {eff }}$ and $r$ indicate that the smoke trail is advected off-axis toward the camera, resulting in an off-axis distance on the same side of the wake as the camera placement. Conversely, a positive $\theta_{\text {eff }}$ implies that the smoke trail is advected off-axis away from the camera. To determine the values of $\theta_{\mathrm{BPA}}$ and $\theta_{\mathrm{PNL}}$ required by Equation (5.15), a simple 2-min average of the wind directions at each tower is used. This is thought to represent an appropriate time scale for spatially determing the location of the wake, as explained in Section 5.3.3. A noticeable increase in the variance of the wind. direction values at the PNL tower became apparent when the wake path encountered the tower. The BPA tower anemometer (59.4 m)

(a) Taken from "MOD-2 Performance, Calculated Using PROP," a table provided by T. R. Richards, NASA-Lewis Research Center, Cleveland, Ohio, 44135. 
indicated a wind direction ranging from $245^{\circ}$ to $260^{\circ}$ during the time the PNL tower ( $260^{\circ}$ from turbine 非 3 ) was in the wake. This observation lends further support to the concept of a mean southerly wake curvature in the time of westerly winds.

\subsection{WAKE HEIGHT DETERMINATIONS}

The photographs of the rocket trails shown in the last section can be used to determine the wind turbine wake height at the frarticular off-axis and downwind positon of the launch pad. Plots of the digitizer data, corrected for lens distortion by the equations of Section 5.3.2 and viewed in comparison to the photographs, provide reliable elevations of particular occurrences in the smoke trail.

Before a detailed analysis can be made of the wake height as deduced from the rocket smoke trails, a suitable example of a smoke trail experiment performed under background conditions is examined. With the turbine not operating, a rocket was launched during the first test period in unstable conditions at the 10 downwind position. The velocity profile that results after correction of the data for lens distortion is shown in Figure 6.5. The abscissa and ordinate are the mean longitudinal velocity in meters per second and the height in meters, respectively. The launch pad was placed at a position sufficiently offset from the turbine to avoid any possible tower wake effect. The wind speed was $6.1 \mathrm{~m} / \mathrm{s}$ and the traverse turbulence intensity about $17 \%$. The smooth, straight profile shows some undulation in the lower region, corresponding to ground turbulence, but generally exhibits a form expected of a profile under no influence of upstream turbine power extraction. Figure 6.5 is generated from a data file having the prefix VELP, denoting the velocity profile data as output from the analysis program. The rocket run number [RTQ4] is appended to the file name. Appendix $C$ examines the details of the file generation and naming structure. The axes of Figure 6.5 are labeled in physical units so that the profile can be easily compared to the runtime observations.

The profiles of several background rockets launched during the course of the field experiments similarly show no gross velocity deficits, nor any large-scale turbulence in the middle-to-upper portions of the profile. This 


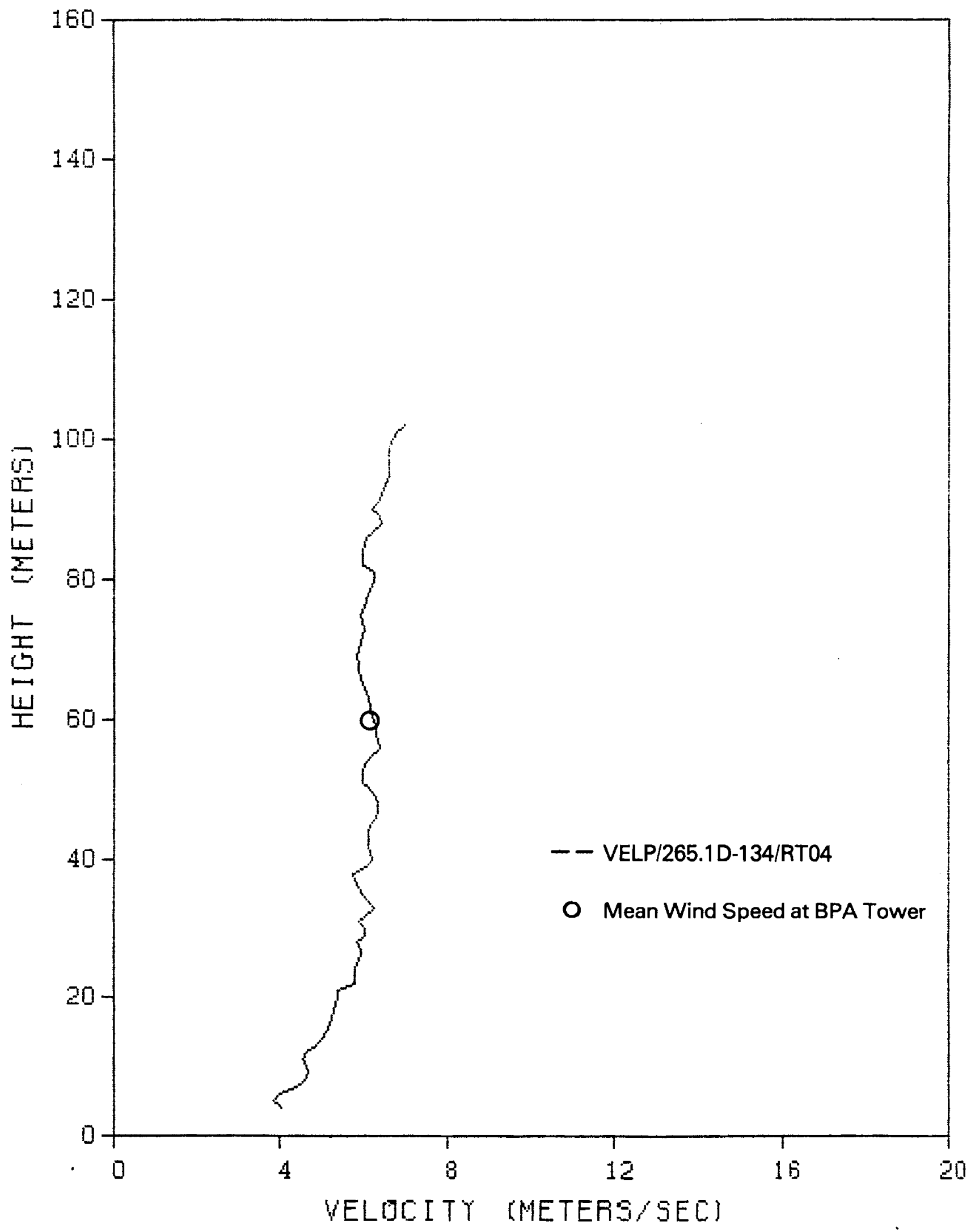

Figure 6.5 Velocity Profile at an Effective Off-Axis Position of -6 Meters (Background) . Launch time: 192:15:30:04. Mean BPA wind speed $=6.1 \mathrm{~m} / \mathrm{s}$. 
fact is very important since in some later cases, a top wake edge is only visible by the interface between the more turbulent wake region and the less turbulent free stream. The reference rocket position plot, corresponding to the velocity profile in Figure 6.5, is shown in Figure 6.6 for examination of the downwind advection of the smoke. Here the abscissa becomes the downwind distance in meters. The file naming structure is similar to that for the velocity profile, except a prefix POSN indicates that the plot is a corrected smoke trail position plot. The time increment between smoke trails, DT, is 0.46 second. Often, it is easier to see the top wake edge from the position plots, comparing them to the photographs, since a velocity deficit is not always discernable from the profile plots. Figure 6.6 will act as a good background reference for all position plots corresponding to times when turbine 非 3 was operating.

A few comments on the features of Figure 6.6 must be made for a thorough understanding of the plot. Each of the traces represents a digitized photograph, and all the sequential traces for this run have been plotted on one graph. The top point of each trace represents the rocket position in the photograph. One may notice that although the rockets typically followed a very straight path vertically, connection of all the first points in each trace of Figure 6.6 does not resemble a straight 1 ine. This is a result of the lens distortion correction corresponding to Equation (5.10), and of the fact that the rocket went out of view after the fourth digitized trail. Also note that the traces toward the right of the plot do not extend all the way to ground level since the smoke has diffused by this point. This has no effect on the velocity profile calculation, since a velocity point is defined only by the average length and number of segments that are encountered in any horizontal cut through the position plot.

Though terrain effects have been mentioned above as possibly accounting for the observed southerly curvature of the wind turbine wake, it was decided to use the observations from a few background rockets at specified terrain locations to compare with all the cases when the turbine was on. Principally, this resulted from the basic observation that all the background rocket velocity profiles deviated less than $10 \%$ from a straight line profile, i.e., a profile having no appreciable shear, except due to ground effects below $25 \mathrm{~m}$. 


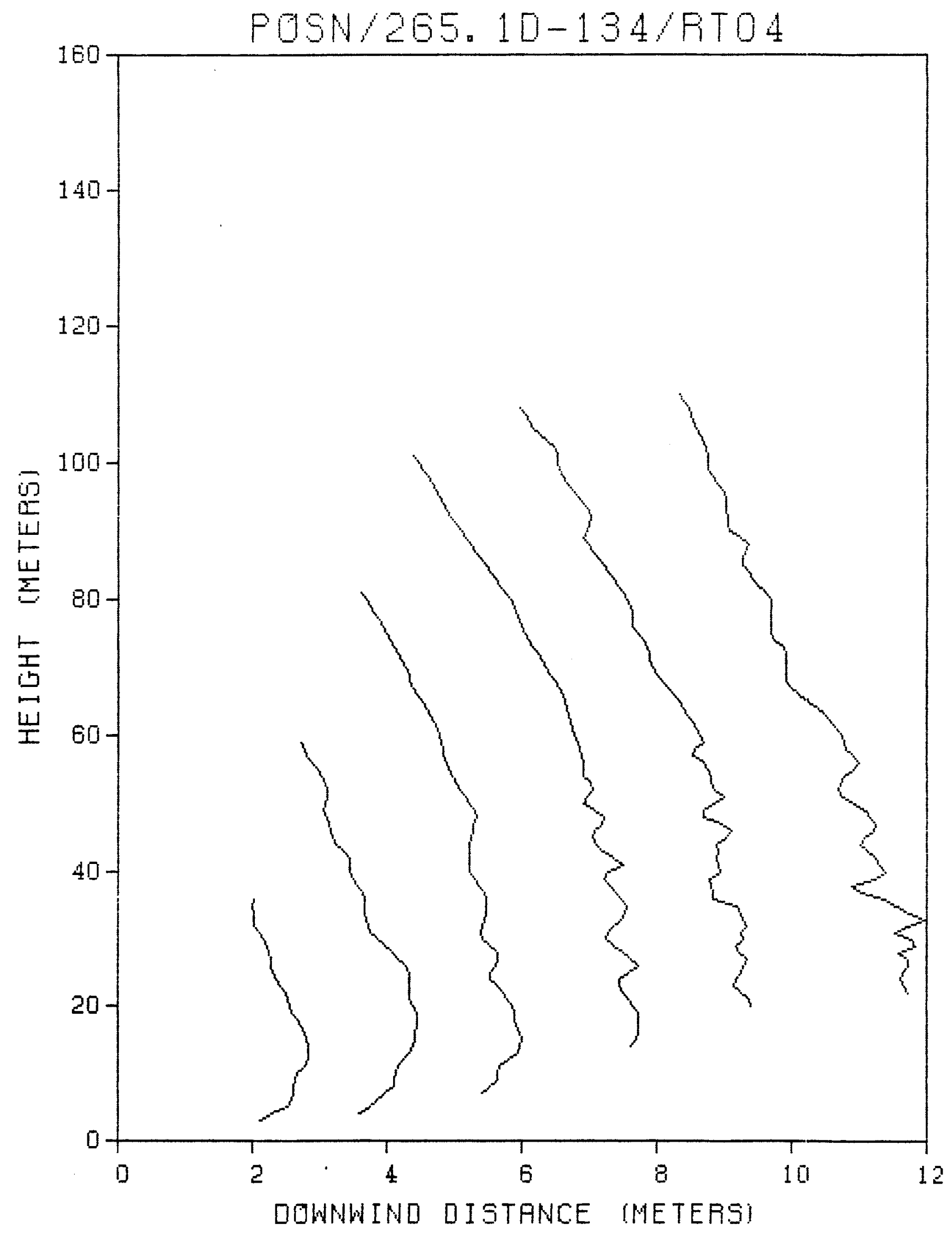

Figure 6.6 Rocket Trail Position Data Corrected for Lens Distortion (Background). Launch time: 192:15:30:04. DT $=0.46 \mathrm{~s}$. 
Using the definition of wake height in which the top edge of the turbine wake is defined as the point at which the velocity deficit vanishes, we may compare the position and profile plots (Figures 6.7 and 6.8 , respectively) of rocket run RT30 with the photographs of Figure 6.2. The smoke trail within the wake shows a very strong shear region having an uppermost vertical extent of $94 \mathrm{~m}$. Though this is even less than the top of the turbine disk $(107 \mathrm{~m})$, the rocket was launched at $12 \mathrm{~m}$ off-axis, so the measurement cannot be directly compared. Later, a plot is developed from all the wake height measurements at any single downwind distance. In this way, a maxinum centerline wake height can be inferred.

The above method of wake height determination is more difficult to implement in the mid-to-far wake, since the shear region associated with the velocity profile may be inconspicuous compared to the background turbulence. In this case the photographs must be examined for the clear demarcation between the turbulent wake and the less turbulent free stream. As an example, the sequence of photographs in Figure 6.4 was taken of rocket run RT66 at a downwind distance of 6D. These can be referenced to position and profile plots (Figures 6.9 and 6.10 , respectively) for determination of the elevation of particular features within the photographs. The photographs show a more rapid diffusion of the rocket trail smoke below an elevation of $150 \mathrm{~m}$, in addition to evidence indicating the existence of a velocity deficit. There is little possibility that the turbulence visible in the photographic sequence of Figure 6.4 is associated with terrain or ground effects. Noting the comparatively smooth traces of the background rocket position plot in Figure 6.6, and observation of the reference rocket in the photographs of the dual rocket launch (Figure 6.2), a distinct difference can be seen in Figure 6.4 between the smoke diffusion rates within and outside of the wake.

Further correspondence between the two methods of determining wake height is indicated by the 2D position and profile plots of Figures 6.11 and 6.12 . In the slightly unstable atmospheric conditions during the early moring of that day in August, the rocket trail distinctly shows an interface between the turbulent MOD-2 wind turbine wake and the more laminar-like free stream. From the position plot, the measured height is $92 \mathrm{~m}$. The analysis of the velocity deficit in the profile plot results in an identical measurement. 


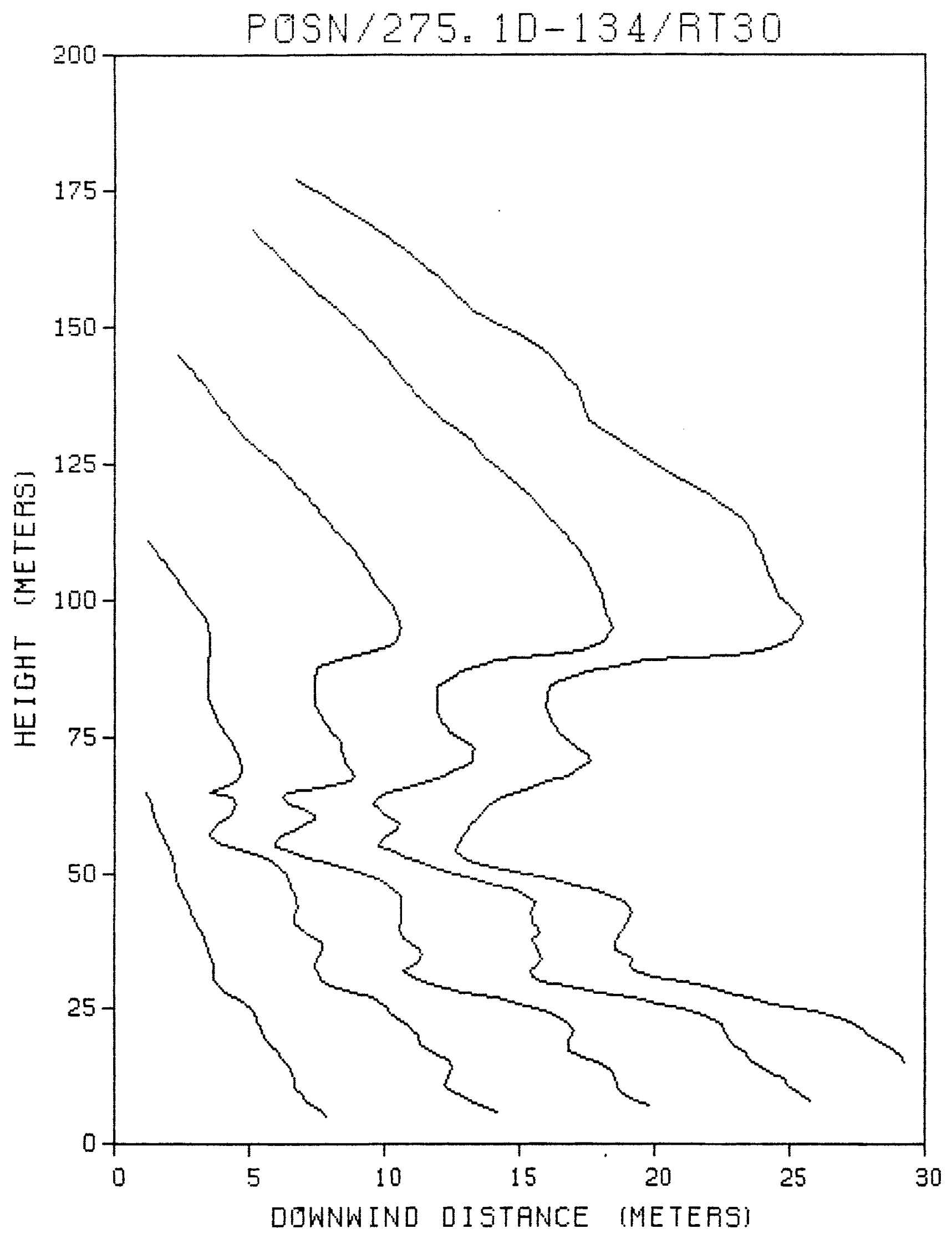

Figure 6.7 Rocket Trail Position Data Corrected for Lens Distortion [RT30] Launch time: $216: 18: 20: 10$. DT $=0.93 \mathrm{~s}$. 


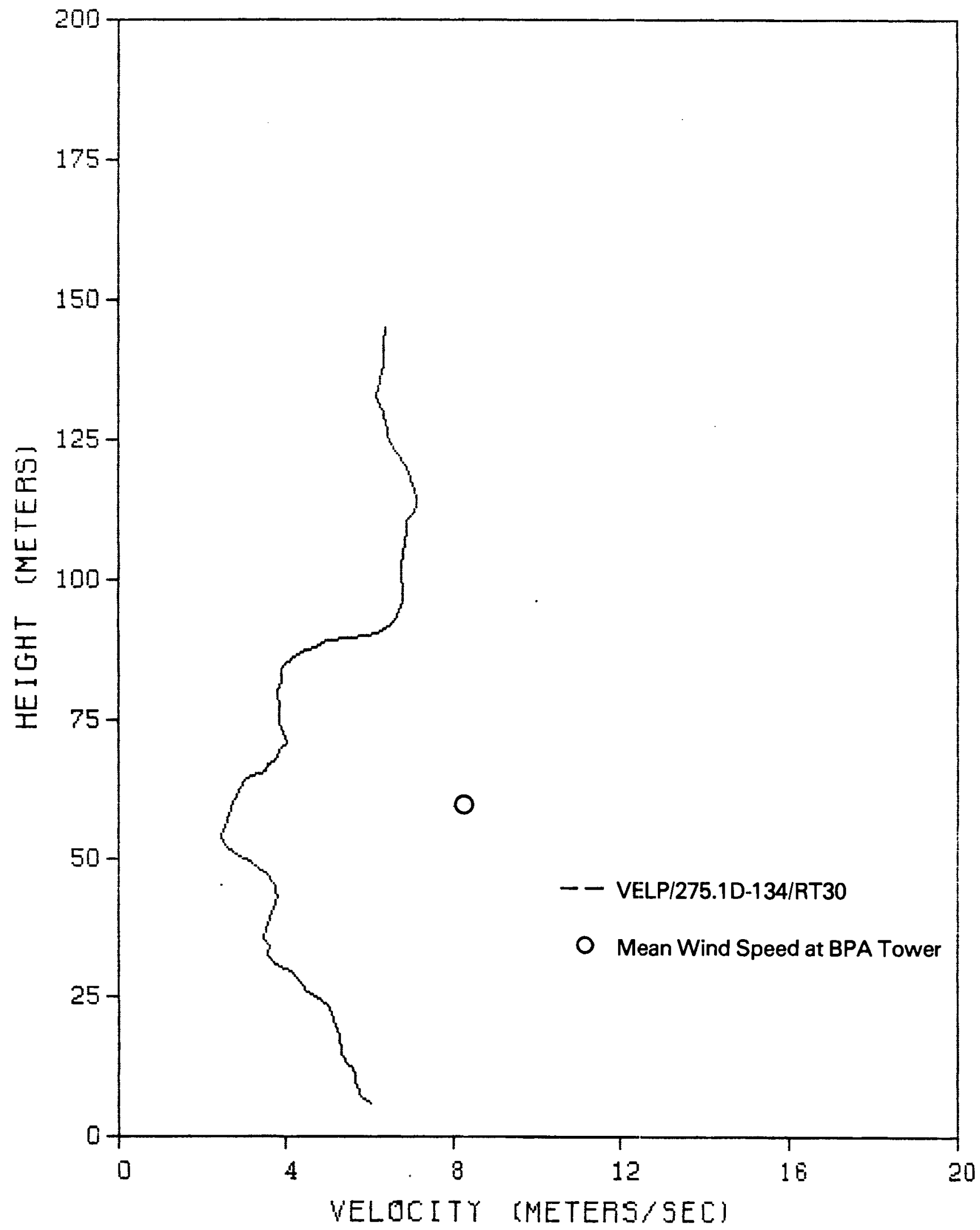

Figure 6.8 Velocity Profile at an Effective Off-Axis Position of +12 Meters [RT30] Launch time: 216:18:20:10. Mean BPA wind speed $=8.2 \mathrm{~m} / \mathrm{s}$. 


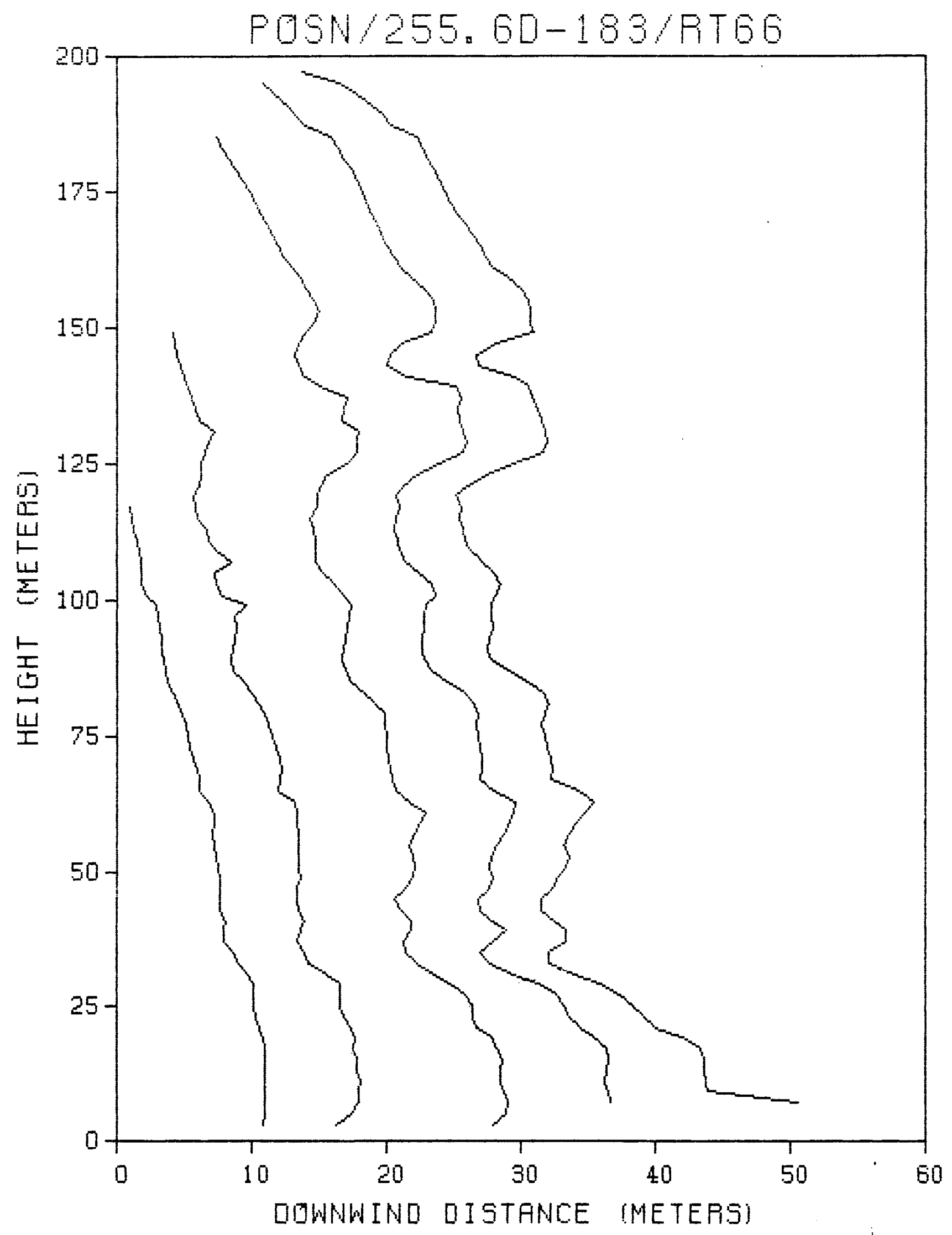

Figure 6.9 Rocket Trail Position Data Corrected for Lens Distortion [RT66]. Launch time: $251: 14: 18: 20 . \mathrm{DT}=0.96 \mathrm{~s}$. 


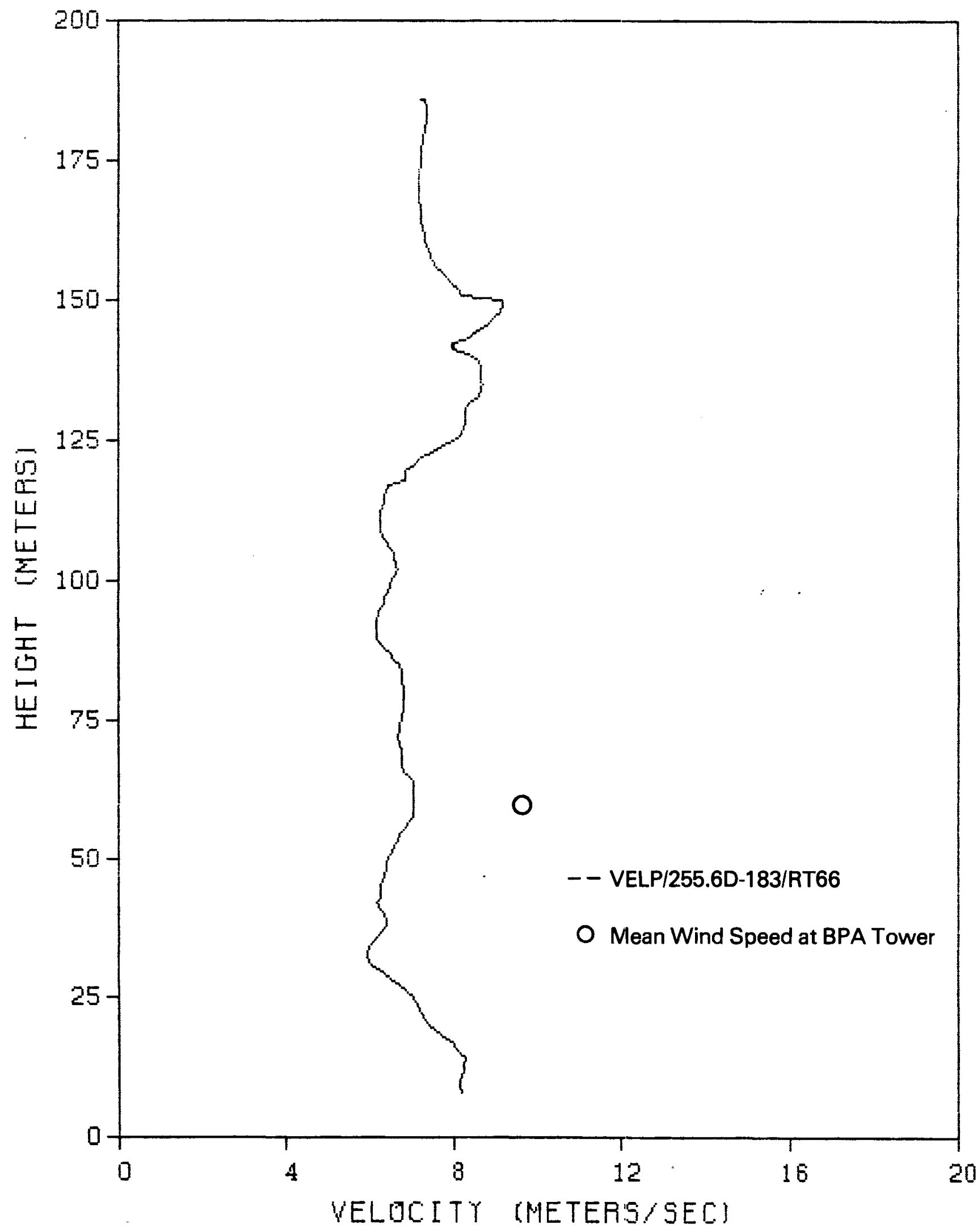

Figure 6.10 Velocity Profile at an Effective Off-Axis Position of -48 Meters [RT66] Launch time: 251:14:18:20. Mean BPA wind speed $=8.6 \mathrm{~m} / \mathrm{s}$. 


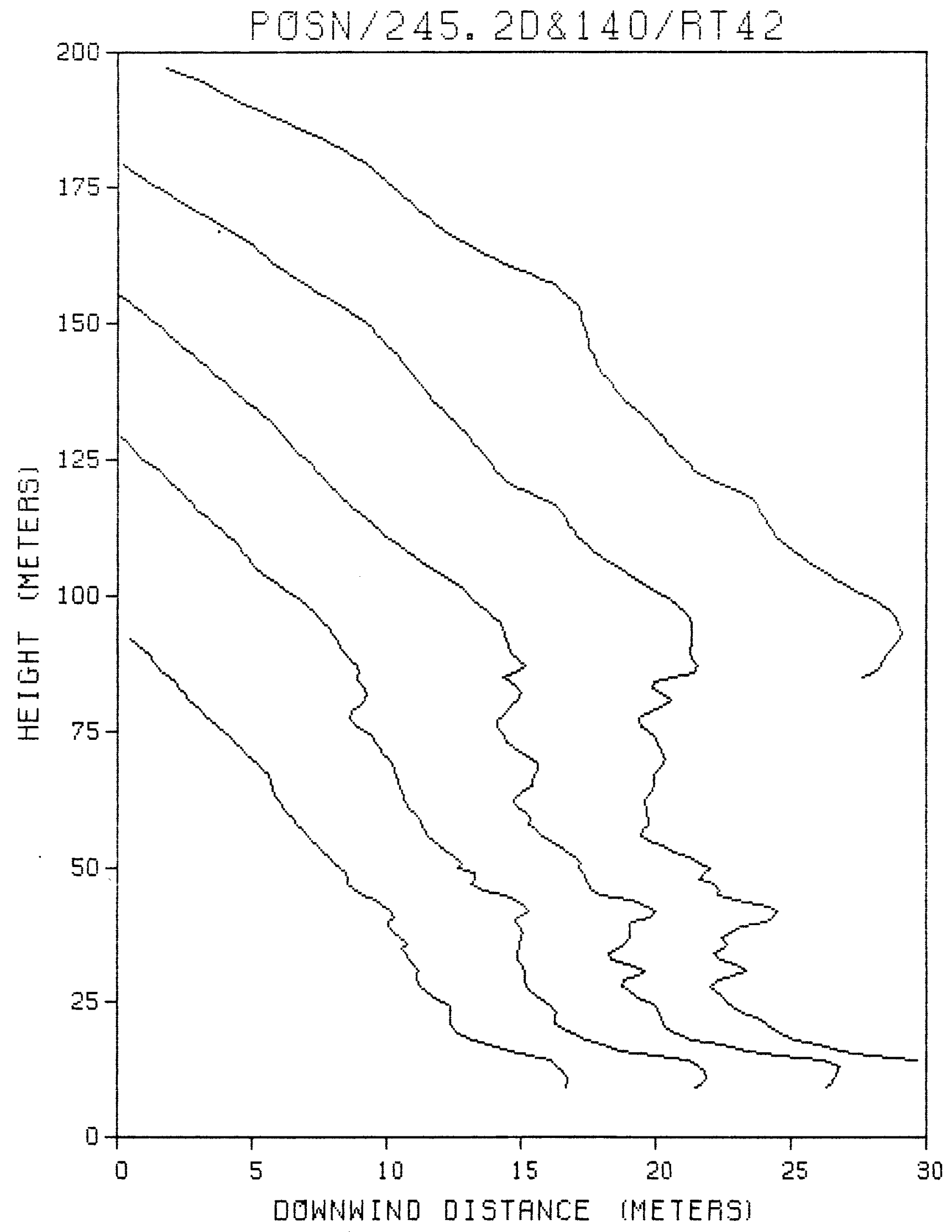

Figure 6.11 Rocket Trail Position Data Corrected for Lens Distortion [RT42] Launch time: $242: 07: 44: 00$. DT $=0.88 \mathrm{~s}$. 


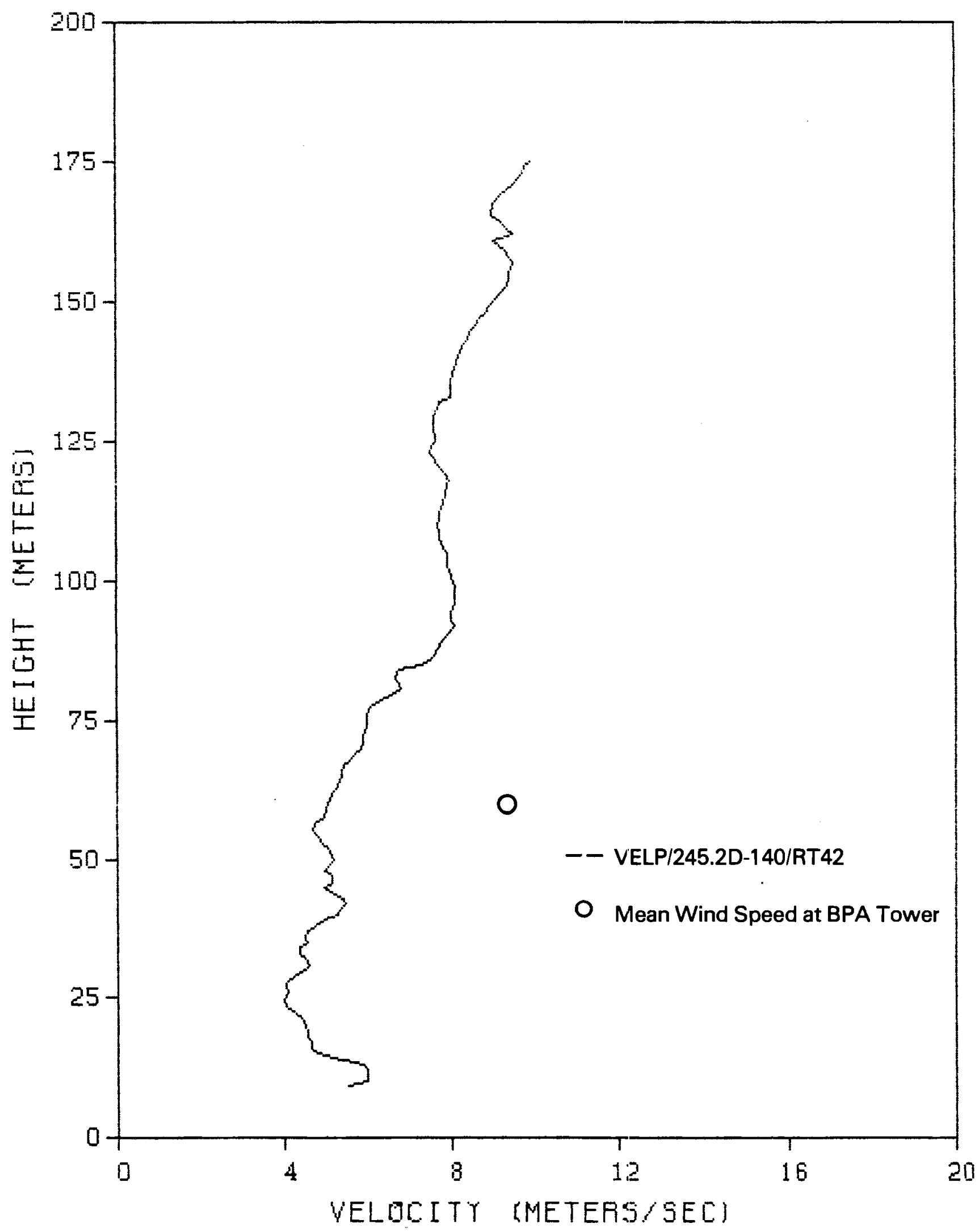

Figure 6.12 Velocity Profile at an Effective Off-Axis Position of +3 Meters [RT42]. Launch time: 242:07:44:00. Mean BPA wind speed $=7.3 \mathrm{~m} / \mathrm{s}$. 
A final velocity profile, which results from the sequence of photographs in Figure 6.1 [RT70], is shown in Figure 6.13 to illustrate the large, uniform velocity deficit encountered at the $1 / 2 \mathrm{D}(46-\mathrm{m})$ downwind position. The measured free-stream velocity of $9.2 \mathrm{~m} / \mathrm{s}$ is very close in agreement to the BPA tower measurement of $9.1 \mathrm{~m} / \mathrm{s}$. The measured wake height $(104 \mathrm{~m})$ exactly corresponds to the value expected $8 \mathrm{~m}$ off-axis if the initial momentum deficit is taken to correspond to the physical turbine disk. This is expected since the wake will have expanded very little during the short advection downwind.

As noted above, highly unstable downwind rocket runs (denoted by the asterisk in Table 6.1) were made under similar conditions and thus can be compared. Figure 6.14 shows a contour plot of the wake height at these positions. The upper wake height measurement in Table 6.1 is plotted against the off-axis distance for each downwind rocket launch position. Though a very small data set exists, there is a trend in the data as indicated by the dashed lines faired through the points for each of 1D, 4D, and 6D to indicate the mean vertical extent of the wake. Note that the single $1 / 2 \mathrm{D}$ point 1 ies exactly on the turbine disk. No linear relationship is made for the two 2D points plotted on Figure 6.14, since too small of a data set exists. These points, along with an unrealistically low wake height measurement at $45 \mathrm{~m}$ [RT47], can only provide knowledge of a range of possible wake height values at the $2 \mathrm{D}$ downwind position.

There is obviously a large degree of scatter in the points plotted in Figure 6.14. This is expected for two reasons. Principally, the interpretation of a few nearly instantaneous and turbulent rocket trail measurements in the time-averaged sense will lead to a large variance in the definition of the mean. Many more single point measurements are needed to accurately define the long-term time-averaged wake position. Secondly, the ambiguity in the determination of $\theta_{\text {eff }}$ causes some scatter in $r$, the off-axis distance. Since the wake is constantly meandering in the horizontal, due to a variance in wind direction, and accentuated by terrain irregularities, the centerline position is difficult to determine instantaneously. Instead of attempting to track the wake centerline, which results from instantaneous changes in wind direction, a 2-min average approach is used, as described in section 5.3.5. 


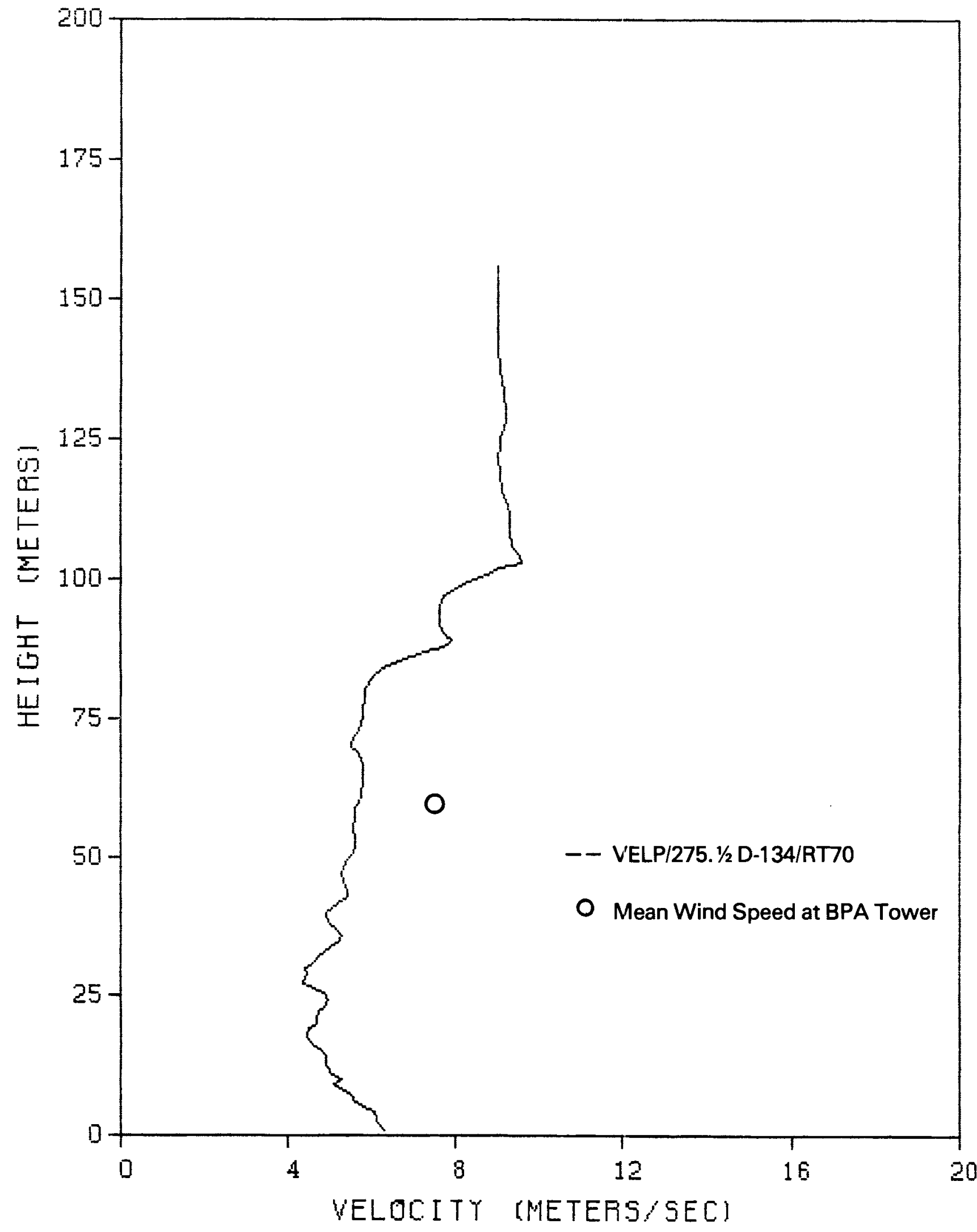

Figure 6.13 Velocity Profile at an Effective Off-Axis Position of -8 Meters [RT70] Launch time: 251:16:12:03. Mean BPA wind speed $=9.1 \mathrm{~m} / \mathrm{s}$. 


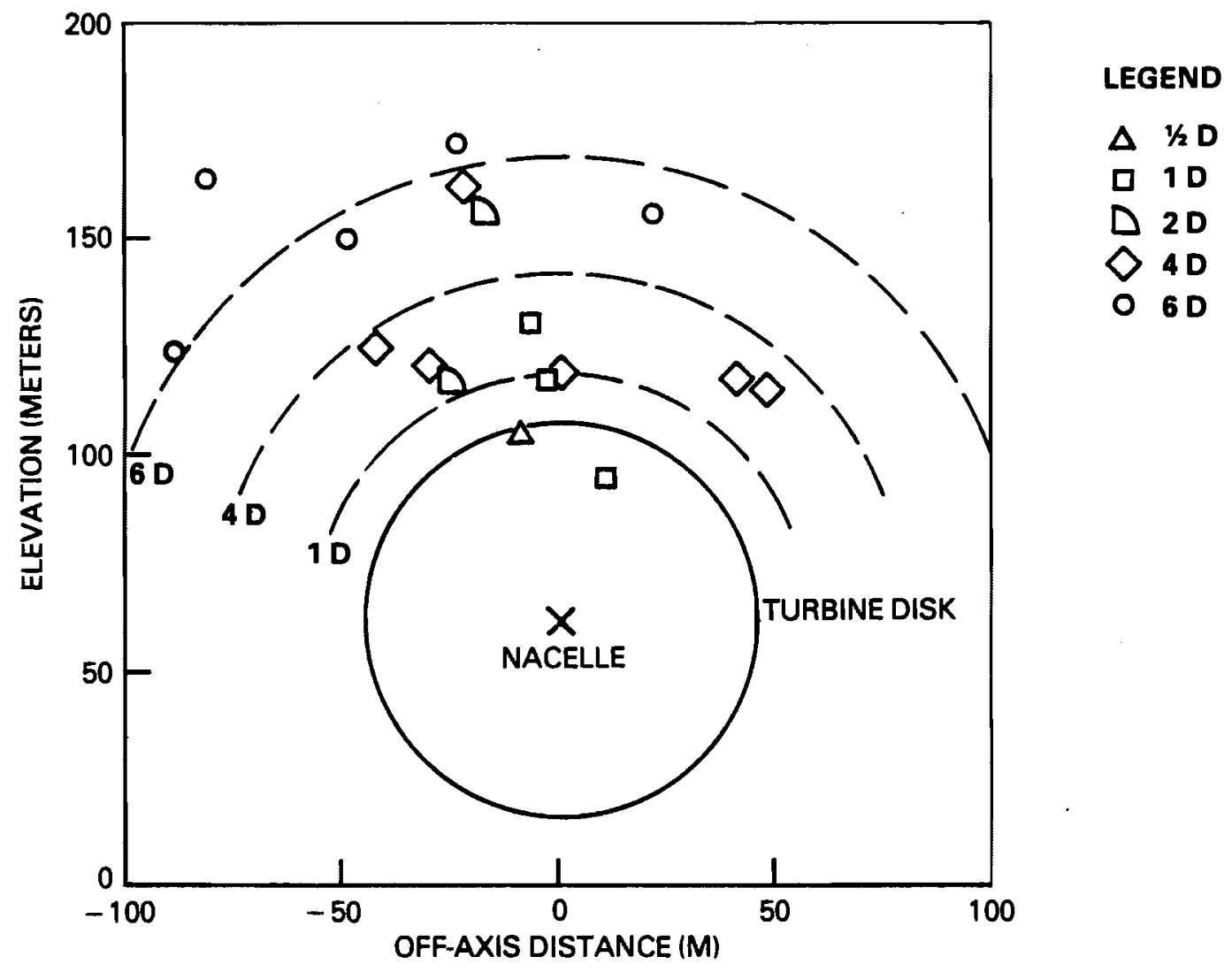

Figure 6.14 Down-Axis View of MOD-2 Wake Growth.

Dashed contour lines indicate the mean wake height at 1D, 4D and 6D downwind. Mean wind speed $=8.2 \pm 1.8 \mathrm{~m} / \mathrm{s}$. Transverse Turbulence Intensity 14\%. 
Nevertheless, the wake height contour plot (Figure 6.14 ) has considerable merit. It is obvious that the random error in $\theta_{\text {eff }}$ described above results in a more systematic error in the determination of wake height, since no wake height can be measured that is larger than the centerline value. Bearing this in mind, the points far off-axis that have heights comparable to the centerline (e.g., the 6D contour) are weighted somewhat less in the placement of the dashed line than are the equally high centerline points.

In addition, the rocket smoke trail visualization of the instantaneous (2-s determination) wake height shows exactly what is viewed in the temporal reference frame of a downwind turbine. The blade periodicity is about 2 seconds, which may be thought of as an appropriate time scale for the assessment of turbulent flow effects on the turbine. Thus, the rocket trail visualization of the flow provides measurements that are more consistent with actual wind turbine behavior than do theoretical models that incorporate the variance in wind direction in their predictions.

\subsection{VELOCITY DEFICIT}

From the velocity profiles presented in the last section, the percentage velocity deficit has been measured for each of the rocket runs shown in Table 6.1. The same criteria apply for comparison of the velocity deficits as for the wake height measurements, so the same runs plotted within Figure 6.14 will also be plotted on a graph of the velocity de:icit versus the off-axis distance.

To understand the analysis procedure, let us consider the photographic sequence of Figure 6.2 and the corresponding position and profile plots (Figures 6.7 and 6.8 ) for the dual $1 \mathrm{D}$ rocket launch of runs RT30 and RT31, having run conditions as detailed in Table 6.1. The strong velocity deficit occurs in the region from 22 to $94 \mathrm{~m}$ in elevation. The turbulence level of the ambient flow during the time of this rocket launch was similar to the background profile of Figure 6.5, thus the segment of filow intersecting the turbine (indicated by the dark line at the left of the photo) can be compared. Evidently, very little vertical shear flow exists in the flow approaching the turbine disk during unstable conditions. Thus the velocity deficit can be determined fairly closely from the velocity profiles by the expression $\Delta U_{m} / U_{o}$, 
where $\Delta U_{m}$ is the difference in magnitude between the measured free-stream velocity on the plot and the "mean internal velocity". Use of this term discriminates between large velocity anomalies within the wake region and the mean value of velocity within the deficit region. For example, at the hub height $(61 \mathrm{~m})$ in Figure 6.8 , a very strong but localized deficit is visible. This may be associated with the turbine nacelle wake, though it does not appear in all $1 \mathrm{D}$ profiles. Since the vertical extent of the total deficit is much greater than the hub height anomaly, the mean internal velocity deficit is calculated using the larger scale structure.

At the 6D downwind position [RT66], the velocity deficit is more difficult to determine from the profile plot of Figure 6.10, ostensibly due to the highly turbulent fluctuations at the top of the wake. An interpretation for the large excess of velocity from 130 to $150 \mathrm{~m}$ could be the existence of large-scale turbulent eddies. Possibly due to tip vortex breakdown, deviations in the local velocity profile may be quite large and, in this case, an excess. If the freestream velocity is taken as $7.7 \mathrm{~m} / \mathrm{s}$ from Figure 6.10 , then a $13 \%$ velocity deficit is evident based on the mean internal velocity determination of $6.7 \mathrm{~m} / \mathrm{s}$.

A strong deficit ( $42 \%)$ is evident from the 2D velocity profile [RT42] shown in Figure 6.12. Because the correction factors become much larger with increasing elevation, the free-stream velocity is taken at the lowest point above the observed deficit. Here there appears to be no large velocity observation as seen in the $6 \mathrm{D}$ profile. In this case, the measured free-stream velocity is $8.0 \mathrm{~m} / \mathrm{s}$, which compares favorably with the BPA tower $59.4-\mathrm{m}$ measurement of $7.3 \mathrm{~m} / \mathrm{s}$.

Based on the results of the velocity deficit determination shown in Table 6.1, a plot can be made of the deficit versus the off-axis distance for the highly unstable runs marked by the asterisk. Again, due to the possibly large errors in the determination of $\theta_{\text {eff }}$, and hence $r$, a large scatter exists in the data. In this case, errors are magnified because the horizontal meandering of the wake affects the velocity deficit measurement, as well as the off-axis distance. This is in contrast to the wake height contour plot, in which higher confidence $c$ an be put into the wake height measurement since relatively little vertical wake meandering will occur. 
The data points for the 4D and 6D downwind positions have such a large variance that only error bars with a midpoint can give light to the timeaveraged velocity deficit. In Figure 6.15, the rectangular box having an initial $\Delta U_{m} / U_{0}$ of approximately $50 \%$ indicates the sharp potential core velocity deficit at the turbine. The lateral dimension of this box is equal to the turbine diameter and results from the single $1 / 2 \mathrm{D}$ rocket launch. (Figure 6.1 verifies that the initial wake has a sharp discontinuity at the turbine disk.) High confidence in the points at $1 D$ has justified the positioning of the dashed 1 ine velocity deficit superimposed at the rectangle. The horizontal extent of this dashed line corresponds to that predicted by the wake height contour plot above (Figure 6.14).

\subsection{COMPARISON WITH A SIMPLE NUMERICAL MODEL}

This section briefly examines the rocket trail ensemble-averaged results for wake height estimates and wake velocity deficit estimates in comparison with the predictions of the Lissaman (Lissaman et al. 1982) model for the wake of a single wind turbine. It must be emphasized that these comparisons are not intended to serve as a definitive verification of the model. The rocket trail results do not constitute a large enough sample for that. Instead, these comparisons are used to check the internal consistency of the rocket trail results and to indicate trends in the data that suggest an inability of simple numerical models to represent very 1 arge wind turbines. The Lissaman model was chosen for two reasons. First, the Lissaman model is easily implemented and used, which means that it is likely to receive a great deal of use in the design of wind turbine arrays in the near future. Second, the Lissaman model is the pioneer of the simple momentum theory approach. As such, it has been compared extensively to other data sets (mostly wind tunnel but some field data) and has spawned a number of similar model attempts by other investigators.

The equations of the updated Lissaman model (Lissaman et al. 1982) were coded to provide vertical profiles of the velocity deficit at arbitrary vertical lines within the wake. That is, the model was capable of producing velocity profiles that correspond to rocket trail launches at arbitrary downwind and off-axis positions in the wake. The comparisons shown here are for vertical profiles through the wake centerline, which was assumed to remain at $61 \mathrm{~m}$ above ground level. 


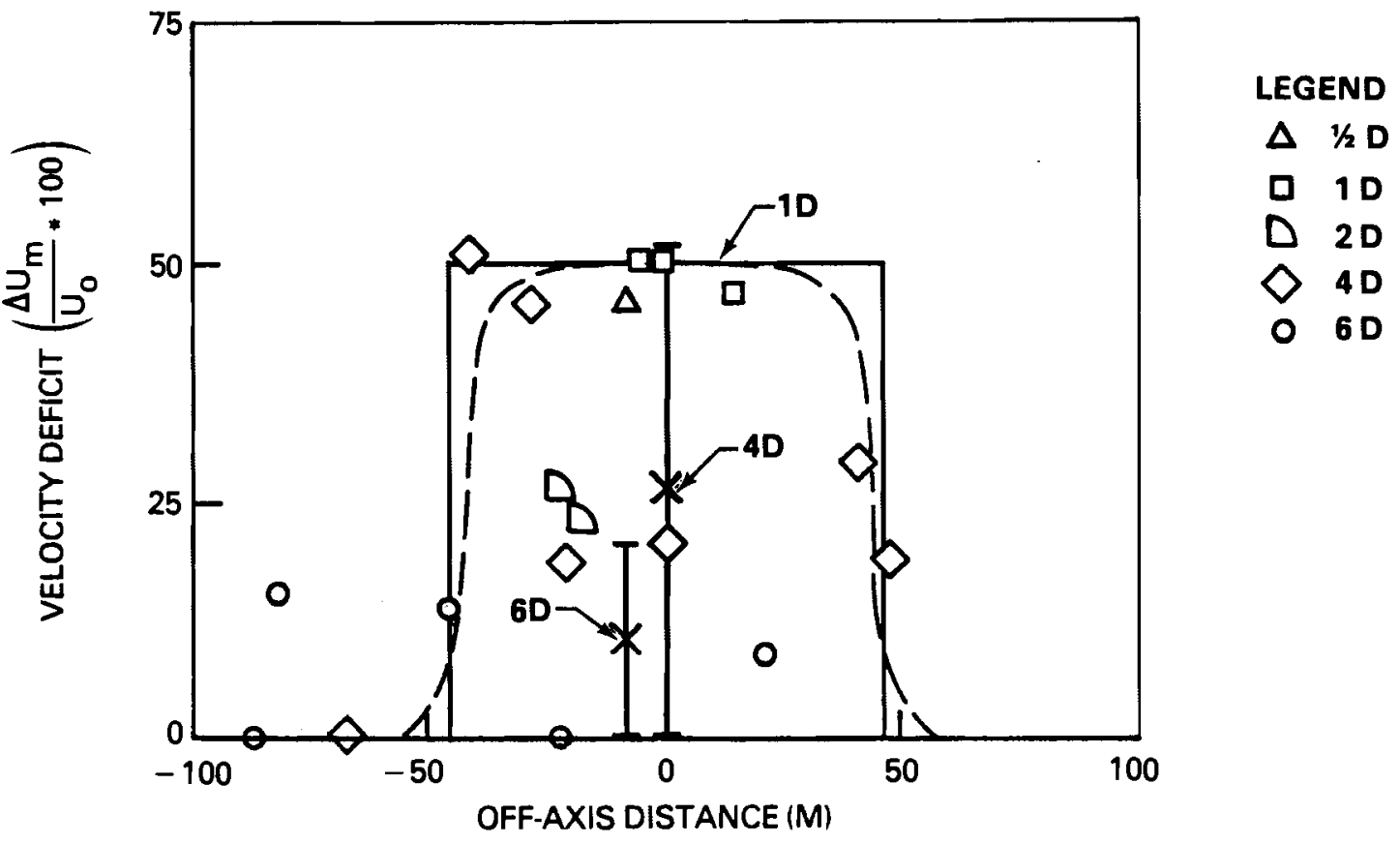

Figure 6.15 Mean Downwind Velocity Deficit Profiles at the 1D, 4D, and 6D Positions . Mean wind speed $=8.2 \pm 1.8 \mathrm{~m} / \mathrm{s}$. Transverse turbulence intensity $\sim 14 \%$. 
Comparisons were made for the data ensemble presented in Figures 6.14 and 6.15. As explained in Sections 6.3 and 6.4 , the conditions for this ensemble were fairly similar for all runs. Notably, all runs were during unstable atmospheric conditions, and all runs were at wind speeds near the peak of the MOD-2 power coefficient curve.

A key input to the Lissaman model is the parameter $m$, where

$$
\mathrm{m}=\frac{\mathrm{U}_{\mathrm{o}}}{\mathrm{u}(\mathrm{x}=0)}=\left(1-\mathrm{C}_{\mathrm{t}}\right)^{-0.5}
$$

$\mathrm{U}_{0}$ equals the free-stream wind speed (assumed the BPA wind speed), $u(x=0)$ is the velocity at the rotor disk, and $c_{t}$ is the turbine thrust coefficient. The mean wind speed measured at the 59-m level of the BPA tower for the ensemble was $8.2 \mathrm{~m} / \mathrm{s}$, with a range from 6.4 to $10.0 \mathrm{~m} / \mathrm{s}$. These wind speeds were used in a table provided by NASA on the performance of the Goodnoe Hills MOD-2 wind turbines to estimate the turbine thrust coefficients. The resulting values of $m$ were in a range from 1.5 (indicating an initial velocity deficit of $33 \%$ ) to 2.0 (an initial deficit of $50 \%$ ) with a value of 1.69 corresponding to the mean wind speed of $8.2 \mathrm{~m} / \mathrm{s}$. Values of $\mathrm{m}$ were also estimated from the turbine power output, but the results were comparable.

The second key input to the Lissaman model is the transverse turbulence intensity. The mean value for the ensemble is $14 \%$; in a range from 10 to $20 \%$.

Figure 6.16 shows three predictions of the maximum wake height versus downwind rotor distance, along with three data points from the rocket trail measurements. The model was run for the measured ambient turbulence conditions $(\alpha=14 \%)$ and for a case of light turbulence conditions ( $\alpha=5 \%)$. The model was also run for the case of wake growth rate $d R_{w} / d x=\alpha / 0.51$, where 0.51 was the constant used in earlier versions of the Lissaman model (see Section 3 ). The model was run for a range of $m$ [Equation (6.2)], but the wake geometry is not sensitive to $m$ over the range considered. The data points represent the apices of the dashed curves in Figure 6.14 .

The model quite obviously overpredicts the wake growth in terms of the growth rate and the wake height. However, we must point out that the comparison may not be valid. On one hand, the wake height determination from Figure 6.14 is something like an ensemble mean of "instantaneous" profiles through the wake. The rocket trails, as pointed out previously, show an abrupt 


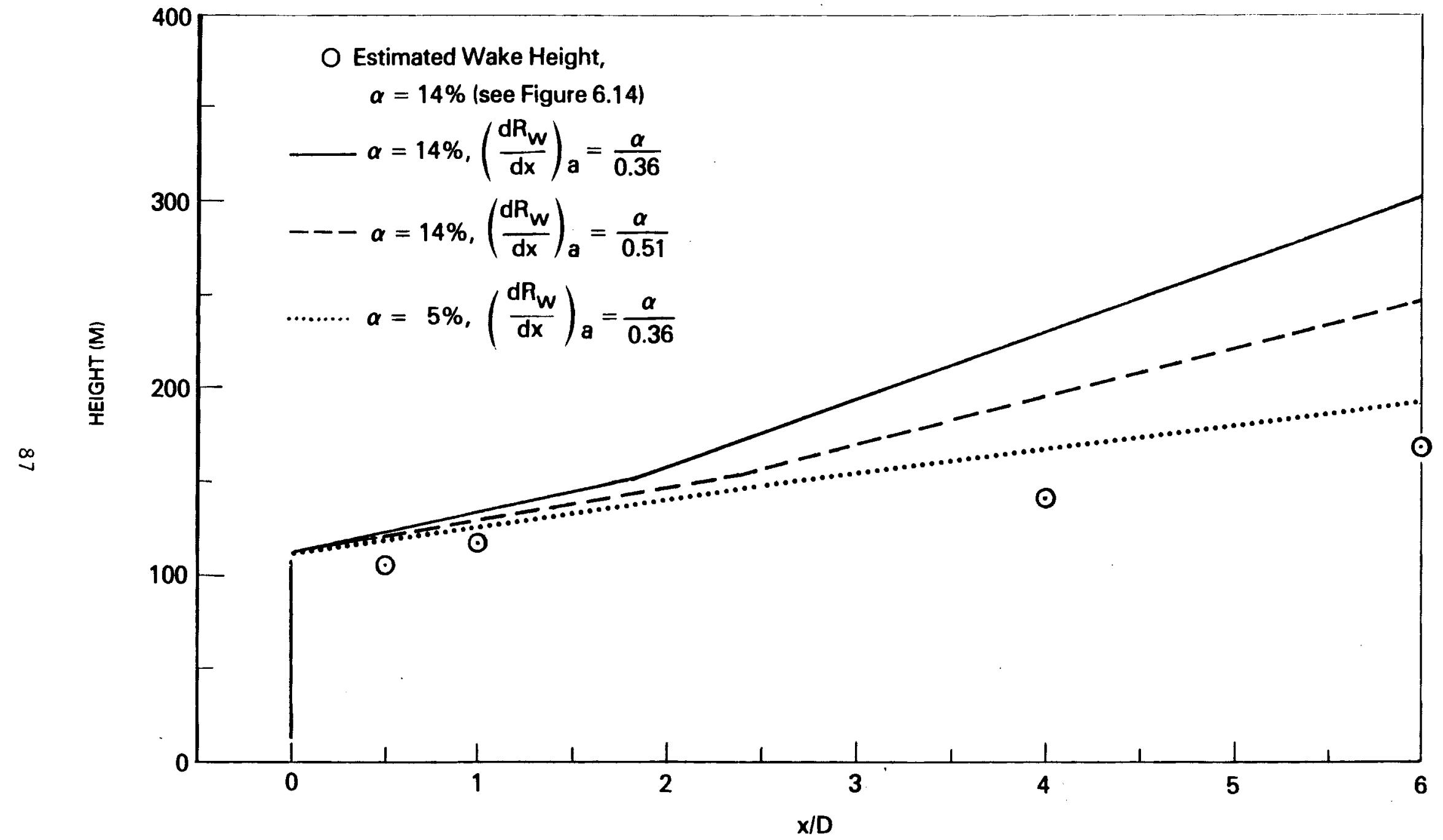

Figure 6.16 Comparison of Lissaman Model and Measurements of Wake Height 
discrete wake boundary. The Lissaman model predicts a discrete boundary also, but the edges of the wake still approach ambient conditions quite smoothly due to the meandering and intermittency of the wake edge in the empirical data upon which the model was founded (see Section 3). The wake model growth rate represents the results of a very large sample of empirical data. It is fair to make the comparison only to the degree that the limited sample of rocket runs represents a significant ensemble. On the other hand, the overprediction by a factor of about 2 at $x=6 \mathrm{D}$ can hardly be accounted for due to wake meander and intermittency alone, especially in the vertical direction. The overprediction in the growth rate, which is relatively sensitive to the difference in the determination of the wake height, indicates that the comparison is quite reasonable.

Assuming that the data of Figure 6.16 can be trusted to some degree, what can be said regarding the comparison with the model? One possibility is that the results are contaminated by topographical effects at the Goodnoe Hills site. It has been observed that there can be a core of higher velocity at about the 60-m level at the PNL meteorological tower. The terrain slopes upward slightly and continuously downwind of turbine 非 (during prevailing wind conditions). A topographically forced acceleration and the associated constriction of streamlines could effect a reduction in vertical wake growth.

A second consideration is that the original coefficient $(0.51)$ for wake growth due to ambient turbulence is better than the new improved one, despite the theoretical justification for the new choice (0.36). Related to this is the observation in Figure 6.16 that the model prediction based on a low ambient turbulence intensity is in better agreement with the data. It may be that the assumptions implicit in the Lissaman model regarding the entrainment process at the wake boundary do not hold in the very large scales considered here. Or, the method of defining the turbulence intensity (or that property of turbulence that affects wake growth) may need rethinking. If these were to be true, since the Lissaman model has been tuned with wind tunnel data (Lissaman et al. 1982), it may indicate the lack of suitability of wind tunnel models to simulate large wind turbine wakes as well.

A second comparison between data and the model is shown in Figure 6.17. In this plot, the range of predictions spanning the range of observed wind speeds 


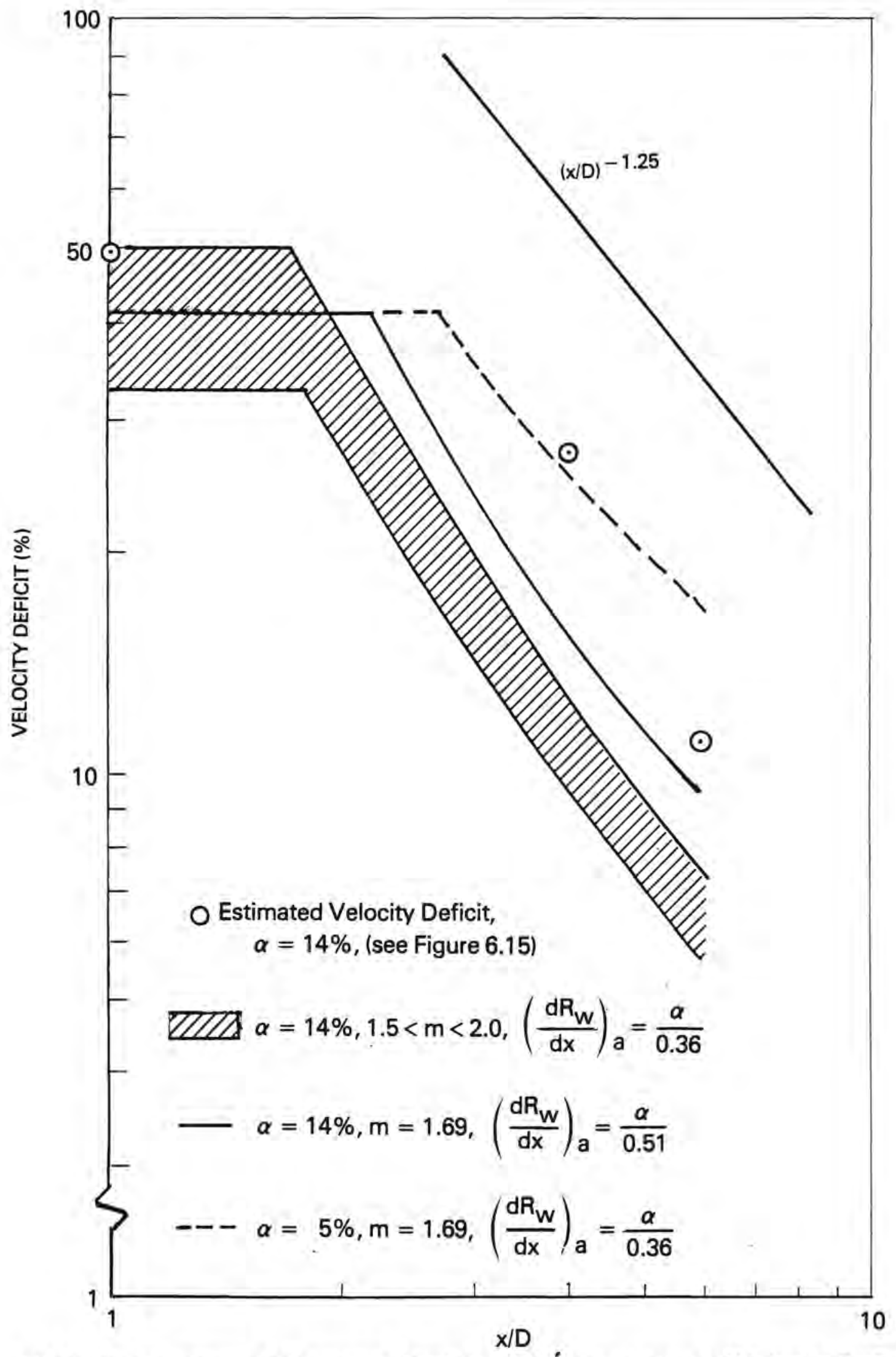

Figure 6.17 Comparison of Lissaman Model and Measurements of Velocity Deficit 
and estimated values of the parameter $m$ is shown. It is seen in general that the model tends to underpredict the mean velocity deficits measured by the rocket technique. Assuming a conservation of momentum deficit, this is consistent with overprediction of the wake growth rate, indicating the wake geometry determinations and the velocity deficit determinations are somewhat interna11y consistent. The result of the comparison is in turn consistent with our qualitative observation that the wake turbulence appears to be lower than we anticipated.

Interpretation of Figure 6.17 indicates the same need for reconsideration of the assumptions of the Lissaman model (and other simple momentum theory mode1s) as discussed above. Is the wake turbulence actually lower than we anticipated? Are these models overpredicting the role of ambient turbulence in wake growth? Is there anything related to the scale of the problem that invalidates the assumptions regarding the behavior and role of wake turbulence? The problem needs further examination since these limited data tend to indicate reduced wake growth rates and higher momentum deficits, thereby indicating a possible need for greater-than-previously-thought spacing between turbines.

\subsection{ROTOR TIP VORTICES}

A discussion of the visualizations of the rotor tip vortices is presented in this section. All three visualization methods, i.e., smoke tracers released from a single point, rocket smoke trails, and flow streamers, were used for observing the tip vortices. These methods complement each other very well, and the integrated results have provided much needed information for understanding the nature of the tip vortices and their possible impacts on other downwind turbines. In the following subsections, the results from the visualizations of the rotor tip vortices using each of these methods are discussed. A summary of the results for the smoke pot and flow streamer experiments is provided in Table 6.2. 
Table 6.2. Summary of Experiments Using Smoke Generators and Flow Streamers

\begin{tabular}{|c|c|c|c|c|c|c|c|c|}
\hline Run & Date & $\begin{array}{l}\text { Time } \\
\text { (PST) }\end{array}$ & $\begin{array}{l}\text { BPA } 59-\mathrm{m} \\
\text { Wind } \\
\text { Speed } \\
(\mathrm{m} / \mathrm{s})\end{array}$ & $\begin{array}{l}\text { BPA } 59-\mathrm{m} \\
\text { Wind } \\
\text { Direction } \\
\text { (degrees) }\end{array}$ & \multicolumn{3}{|c|}{$\begin{array}{c}\text { Coordinates of Smoke Sources } \\
\text { or Flow Streamers }\end{array}$} & Comments \\
\hline $\operatorname{SP} 17(a)$ & $8 / 3 / 82$ & $17: 34: 00$ & 6.3 & 297 & 11 & 37 & 27 & \\
\hline SP22 & $8 / 4 / 82$ & $15: 41: 00$ & 7.8 & 267 & 11 & 37 & 27 & \\
\hline SP 24 & $8 / 4 / 82$ & $16: 10: 00$ & 7.1 & 267 & 11 & 37 & 27 & \\
\hline SP25 & $8 / 4 / 82$ & $16: 33: 00$ & 6.8 & 268 & 11 & 37 & 27 & \\
\hline FS36 (b) & $8 / 5 / 82$ & $18: 45: 00$ & 8.6 & 275 & 180 & In-Wake & 280 & $\begin{array}{l}\text { 180-m Line Length }\left(40^{\circ}(\mathrm{c}) \text { ) }\right. \\
\text { Crepe Paper Streamers }\end{array}$ \\
\hline FS73 & $9 / 10 / 82$ & $11: 00: 00$ & 8.4 & 252 & 45 & 45 & 26 & $\begin{array}{l}120-\mathrm{m} \text { Line Length }\left(60^{\circ}\right) \\
\text { Fabric Streamers }\end{array}$ \\
\hline FS75 & $9 / 10 / 82$ & $13: 16: 00$ & 10.5 & 256 & 370 & In-Wake & - & $\begin{array}{l}200-m \text { Line Length }\left(60^{\circ}\right) \\
\text { Fabric Streamers }\end{array}$ \\
\hline FS76 & $9 / 10 / 82$ & $14: 30: 00$ & 10.3 & 266 & 370 & Ambient & - & $\begin{array}{l}200-\mathrm{m} \text { Line Length }\left(60^{\circ}\right) \\
\text { Fabric Streamers }\end{array}$ \\
\hline
\end{tabular}

\footnotetext{
(a) $\mathrm{SP}=$ Smoke Pots

(b) FS $=$ F1ow Streamers

(c) Angle of Inclination to the Horizontal
} 


\subsubsection{Visual Results from Point Release of Smoke Tracers}

Several trial runs were conducted before an optimum placement of the smoke pot was found. During each run, the tethered cable was 1 ined up almost parallel to the plane of the blades with the smoke pot placed about $0.12 \mathrm{D}$ downwind of the blades (see Figure 5.6). From the optimum placement of the smoke pot, we determined that the maximum strength of the tip vortices was very close to the tip of the blades. For an airplane, the trailing vortex pair is initiated at the wing tip and the vortex separation approaches an asymptotic value smaller than the wing span. According to inviscid wing theory, the asymptotic separation of a trailing vortex pair generated by a large aspect ratio wing is $(\pi / 4) \mathrm{S}$ or $0.79 \mathrm{~s}$, where $\mathrm{S}$ is the span of the wing (Thwaites 1960). For a wind speed of $10 \mathrm{~m} / \mathrm{s}$ and a rotational speed of $17.5 \mathrm{rpm}$ or a tip speed of $83.8 \mathrm{~m} / \mathrm{s}$, the total effective angle of attack of the rotor blades is $-13.8^{\circ}$, with the tips feathered at $-5^{\circ}$.

To help interpret the results of flow visualization, in Figure 6.18 we sketch the side and front views of the trajectories of the rotor tip vortices. The helical trajectories may be viewed as a stretched "slinky" with the solid and dashed portions representing the near and far halves of the slinky, respectively. In the figure, the direction of a tip vortex is indicated by a dot (out of the paper), a plus (into the paper), or an arrow. The sense of rotation of the trajectory is illustrated by small ellipses or circles. From the sense of the rotation, we find that an induced velocity of the helical trajectories forces them to move toward the turbine, whereas the mean wind advects the trajectories downwind. The net effect is to cause the trajectories to move more slowly than the ambient wind.

Figures 6.19 and 6.20 show the side view of several tip vortex trains for runs SP24 and SP17. The 10-min averaged wind speeds are 7.1 and $6.3 \mathrm{~m} / \mathrm{s}$, and the corresponding wind directions are $267^{\circ}$ and $297^{\circ}$, respectively, as measured from the sensors on the BPA tower at the 59.4-m level. These pictures, which sequentially run up and down (and from left to right in Figure 6.19), were photographed at a speed of 3.5 frames/second. Note that the pictures in Figure 6.20 are reversed in color, an attempt to enhance the contrast between the smoke and the background. The smoke pot was placed at the south wake edge at about $11 \mathrm{~m}$ downwind of the rotor, $27 \mathrm{~m}$ above the turbine base, and $37 \mathrm{~m}$ away 


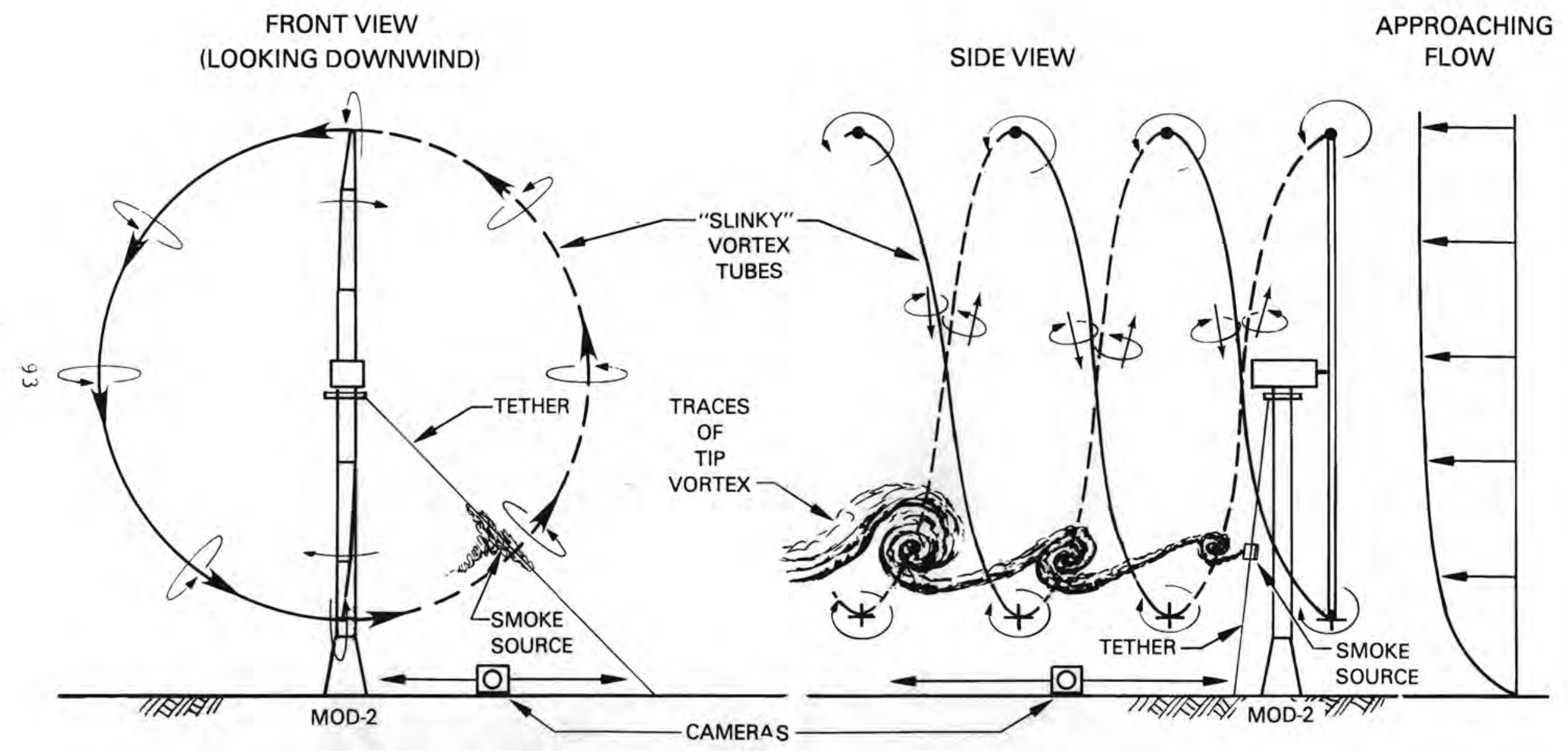

Figure 6.18 Conceptual Drawing of the Rotor Tip Vortices with Relation to the Smoke Observation . Solid and dashed lines represent, respectively, the near and far halves of the vortex tubes. 


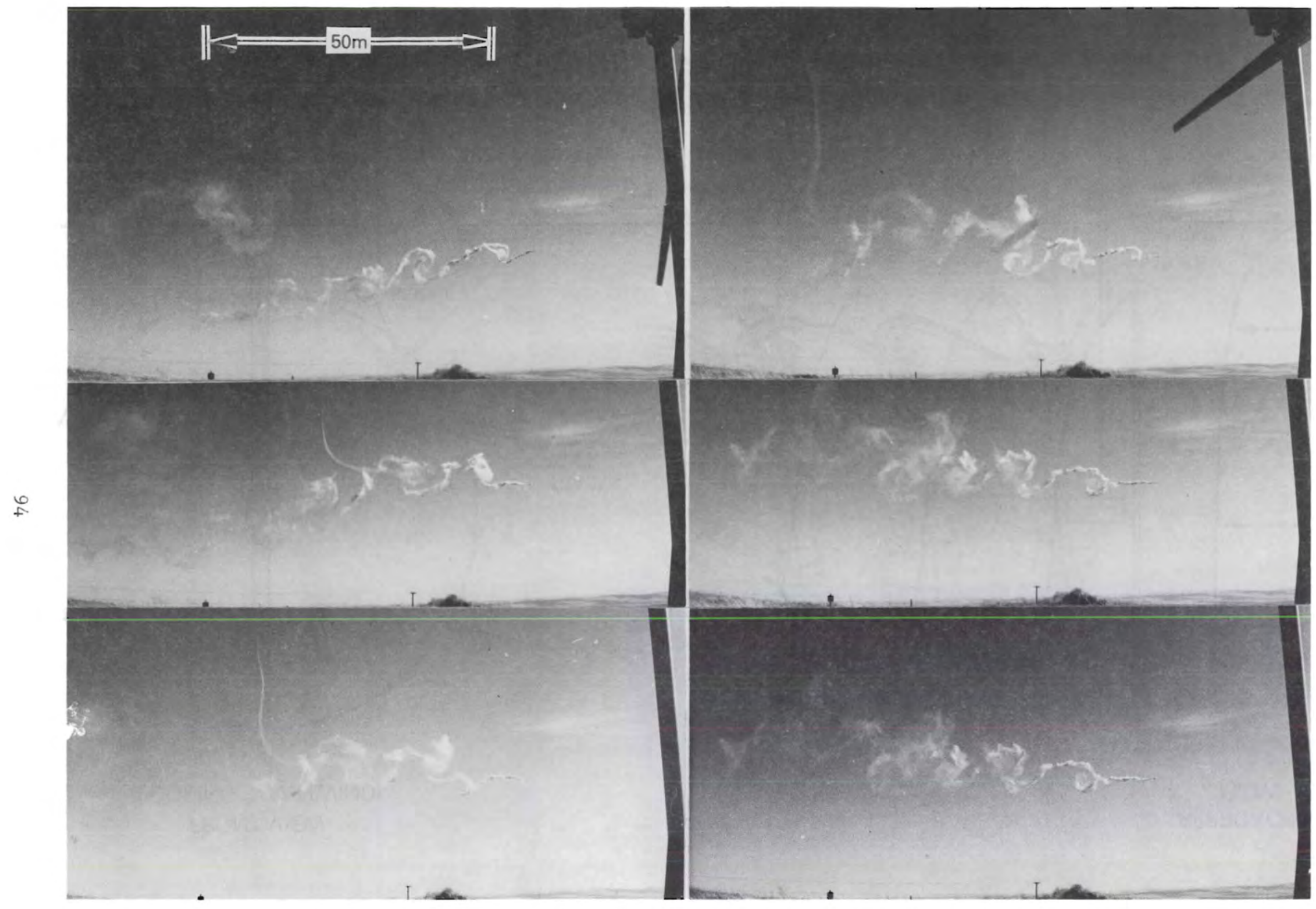

Figure 6.19 Side View of Rotor Tip Vortices [SP24]

The advection of a reverse spout is clearly observable. The length of the spout is estimated to be about $100 \mathrm{~m}$. The time between pictures is about 0.3 seconds. 

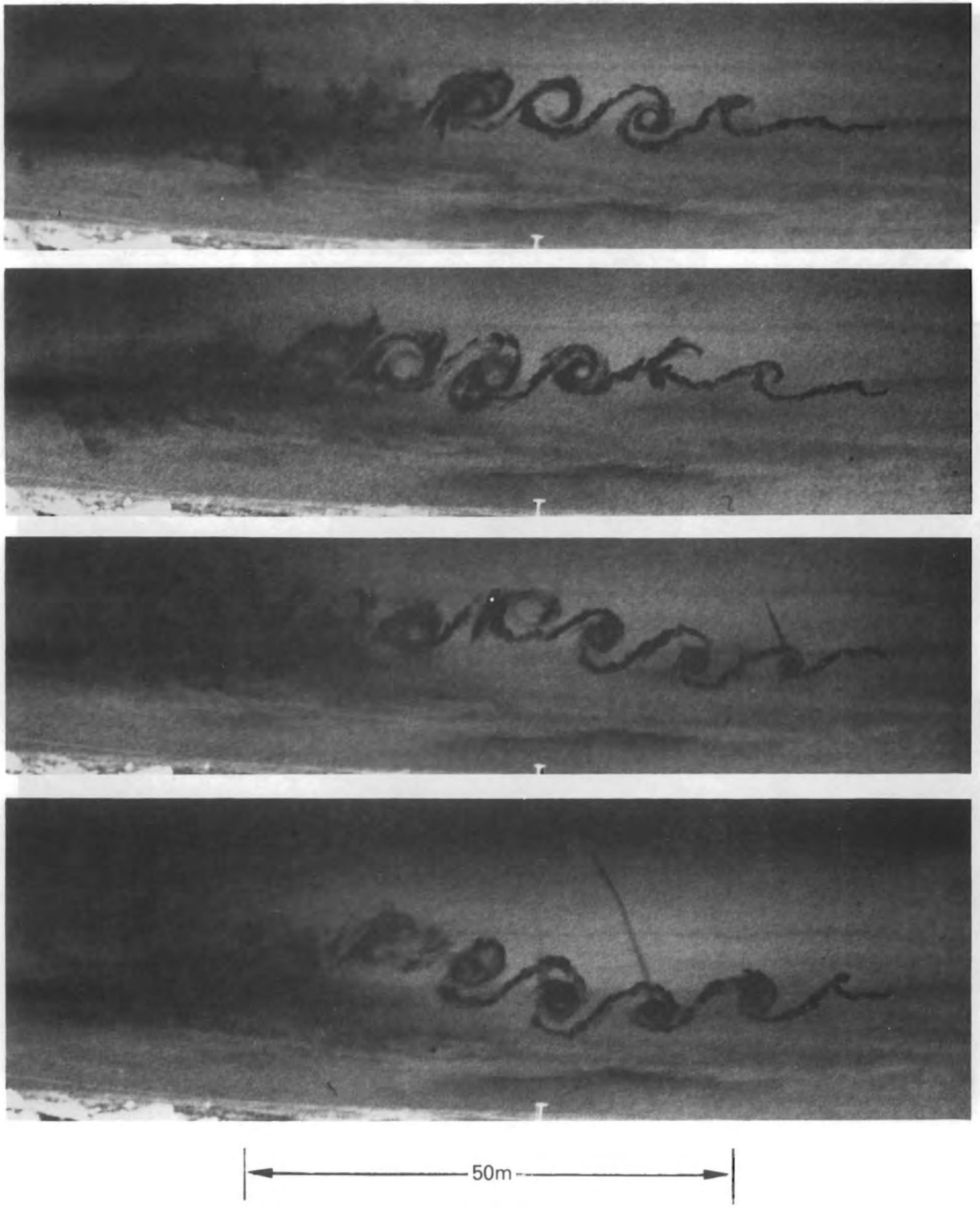

Figure 6.20 Side View of Rotor Tip Vortices That Persist as Far as 1.5 D [SP17] The pictures were taken looking south, and the time between pictures is about 0.3 seconds. Many reverse spouts are observed. The axial speed of the spouts is estimated as high as $50 \mathrm{~m} / \mathrm{s}$. 

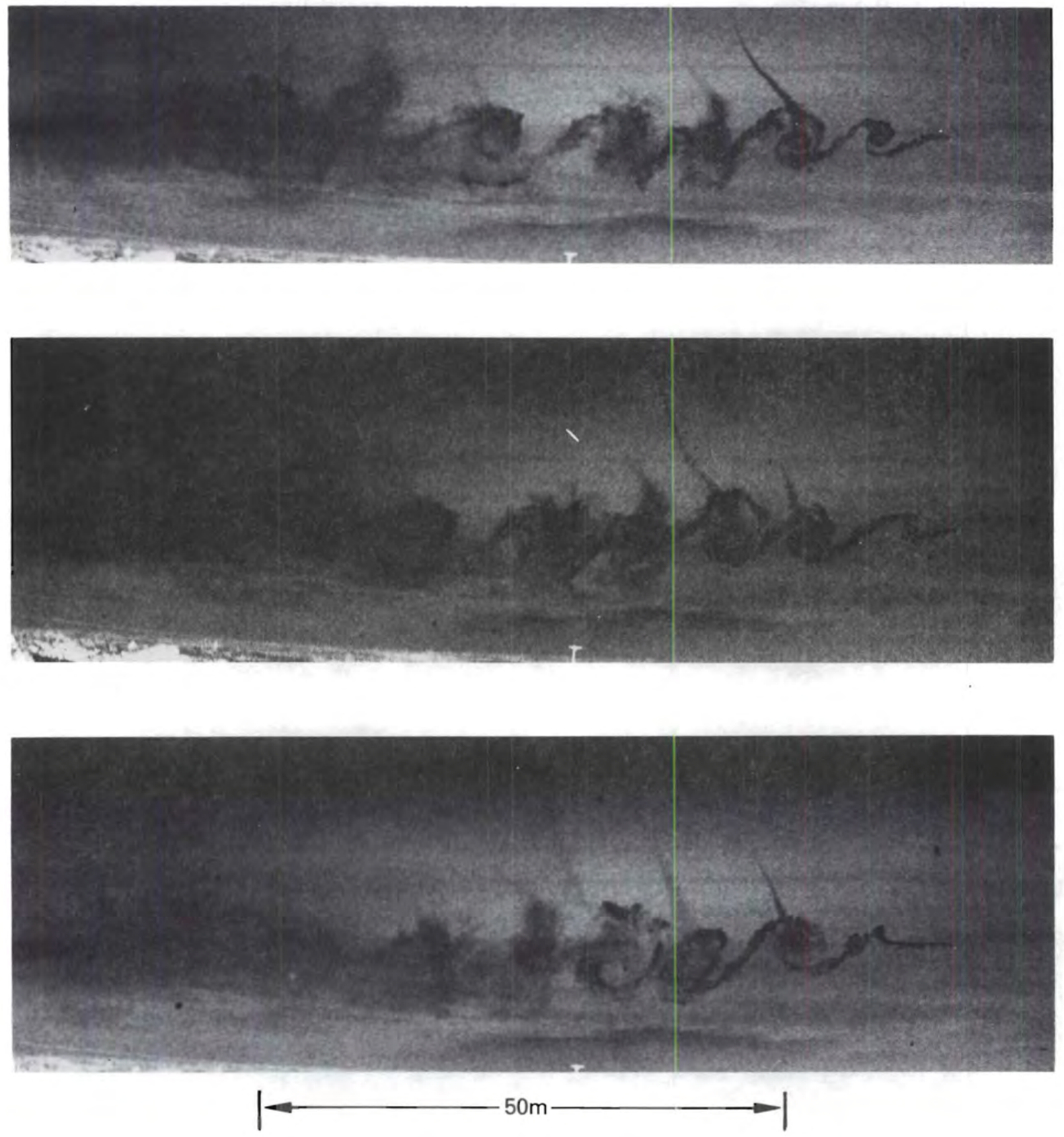

Figure 6.20 Side View of Rotor Tip Vortices That Persist as Far as 1.5D (Concluded). 
from the turbine tower. The camera was placed on the north side of the turbine, and the length scale along the plane of the vortices is given in the figure. As a whole, each rotor blade generates a tip vortex every time it sweeps by the smoke pot. However, such is not the case when the smoke pot is not placed in the optimum position. In addition, the unsteadiness of the wind may occasionally cause the vortices to miss the smoke source. The tip vortices in Figure 6.20 are more organized and persistent than those in Figure 6.19. It should be pointed out that the smoke tracer is not a good indicator for persistence of the vortices at long distances because of its fast diffusion rate. The trains of vortices may be advected upward or downward by the ambient wind as can be observed from the movie record. The mean separations between the vortices are estimated to be about $11 \mathrm{~m}$ in Figure 6.19 and $10.3 \mathrm{~m}$ in Figure 6.20 . The corresponding advecting speeds are therefore about 6.4 and $6.0 \mathrm{~m} / \mathrm{s}$, as compared with the 10 -min averaged speeds of 7.1 and $6.3 \mathrm{~m} / \mathrm{s}$. The relatively slow advection of the vortices is consistent with the direction of the induced velocity of the helical vortex trajectories, as illustrated in Figure 6.18.

of most interest is the presence of spouts that are generated within the cores of the vortices, as observed from Figures 6.19 and 6.20. To illustrate the details of the generation and evolution of the spouts, we present in Figures 6.21 through 6.24 the front view (looking downind) of selected spout configurations. For this particular perspective angle, the blades are to the left of the pictures and they rotate toward the ground. Figure 6.21 shows two "forward" and two "reverse" spouts that move in the same and in the opposite directions as the rotation of the blades, respectively. Details of the forward and reverse spouts are displayed in enlarged pictures taken with zoom lenses (Figures 6.22 and 6.23).

From all the experimental results gathered on the tip vortices, including the movie records (Liu et al, 1982), we are able to provide a description of the generation and evolution of the tip vortices and the spouts. At times, a forward spout that follows the direction of the blades is observed after passage of a blade (Figures 6.19 and 6.20). A few of the forward spouts undergo a sudden reversal in their direction shortly after they travel a short distance. The flow reversal takes place within the vortex cores, that is, the core diameter of the reverse spout is smaller than that of the forward spout. 

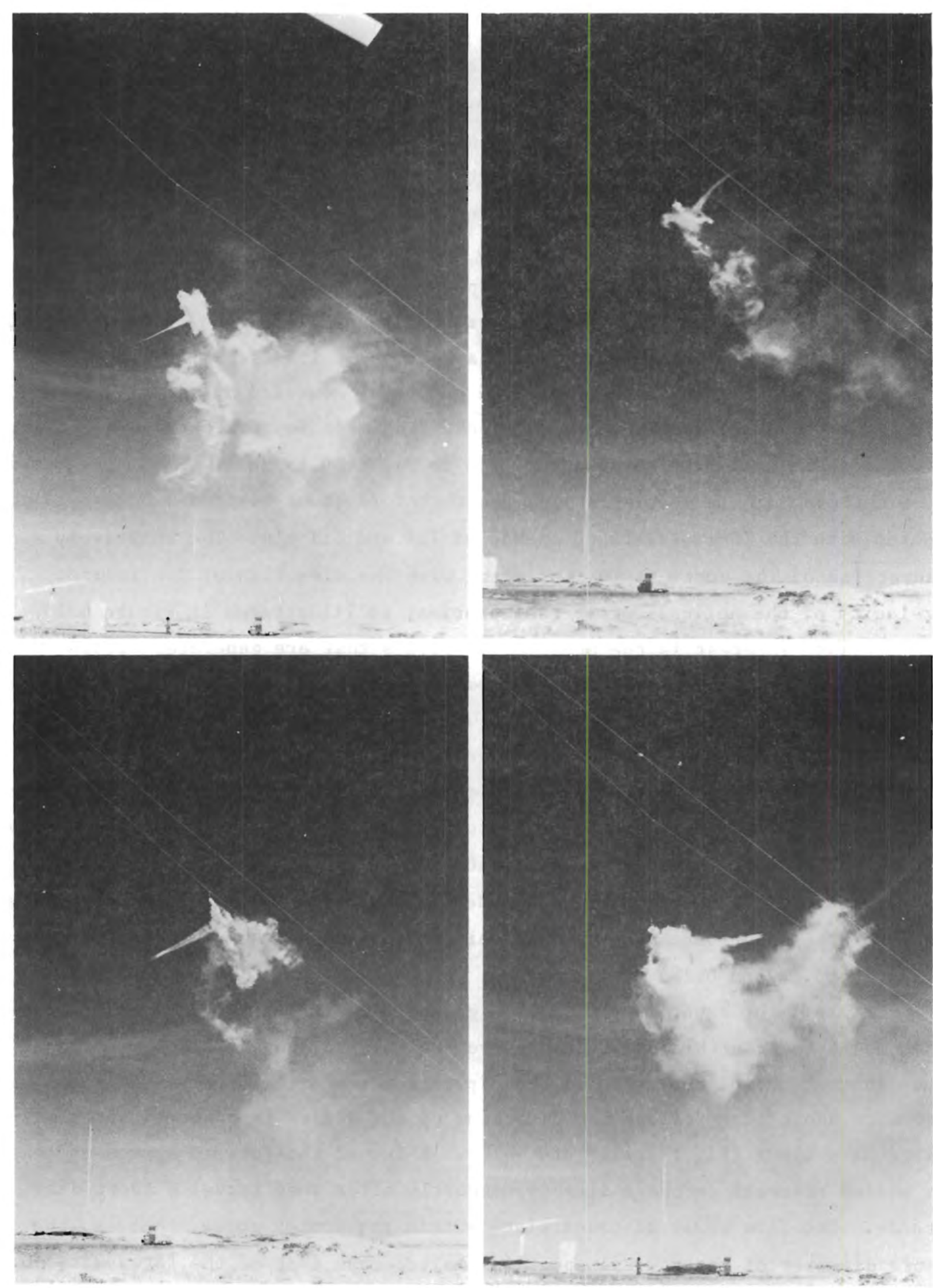

Figure 6.21 Downwind Views of the Tip Vortices [SP24] .

The pictures on the left show the forward spouts and those on the right, the reverse spouts. The elevation of the pulley above the smoke pot is about $25 \mathrm{~m}$ 

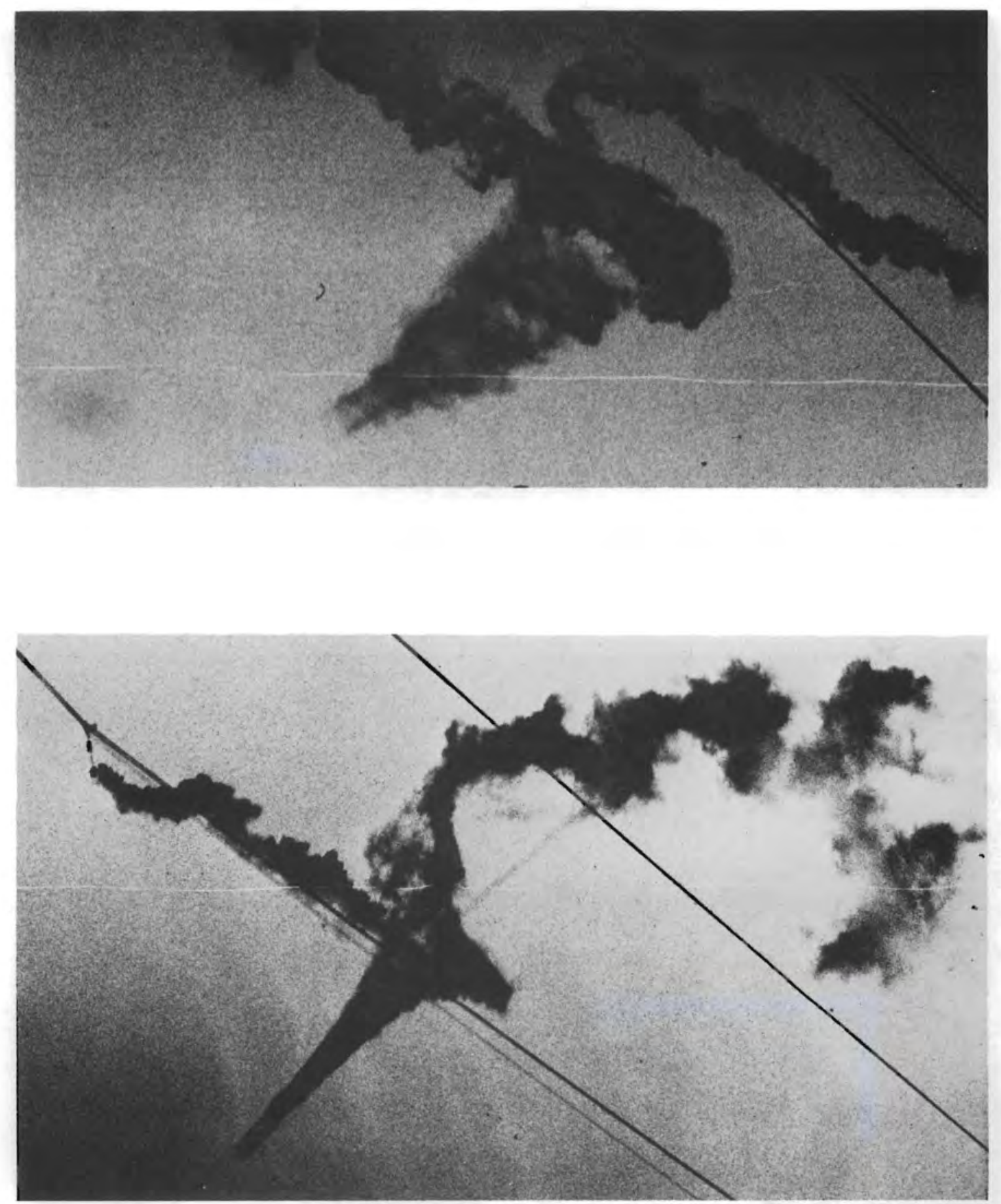

Figure 6.22 Closeup of Forward Spouts [SP24]

The pictures were taken looking downwind. The separation of the tethered lines is about $2.5 \mathrm{~m}$. 

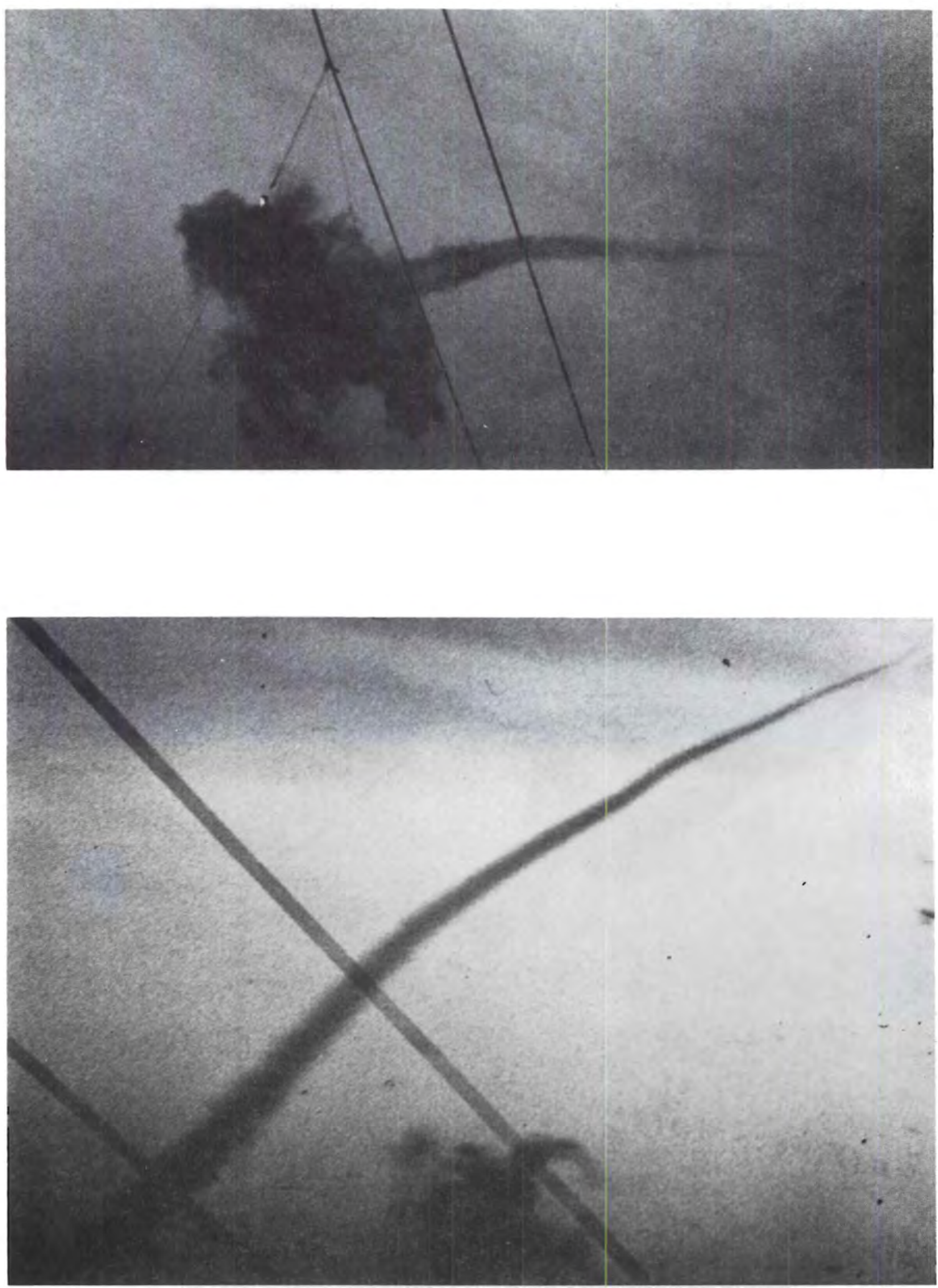

Figure 6.23 Closeup of Reverse Spouts [SP22] .

The pictures were taken looking downwind. The separation of the tethered lines is about $2.5 \mathrm{~m}$. 

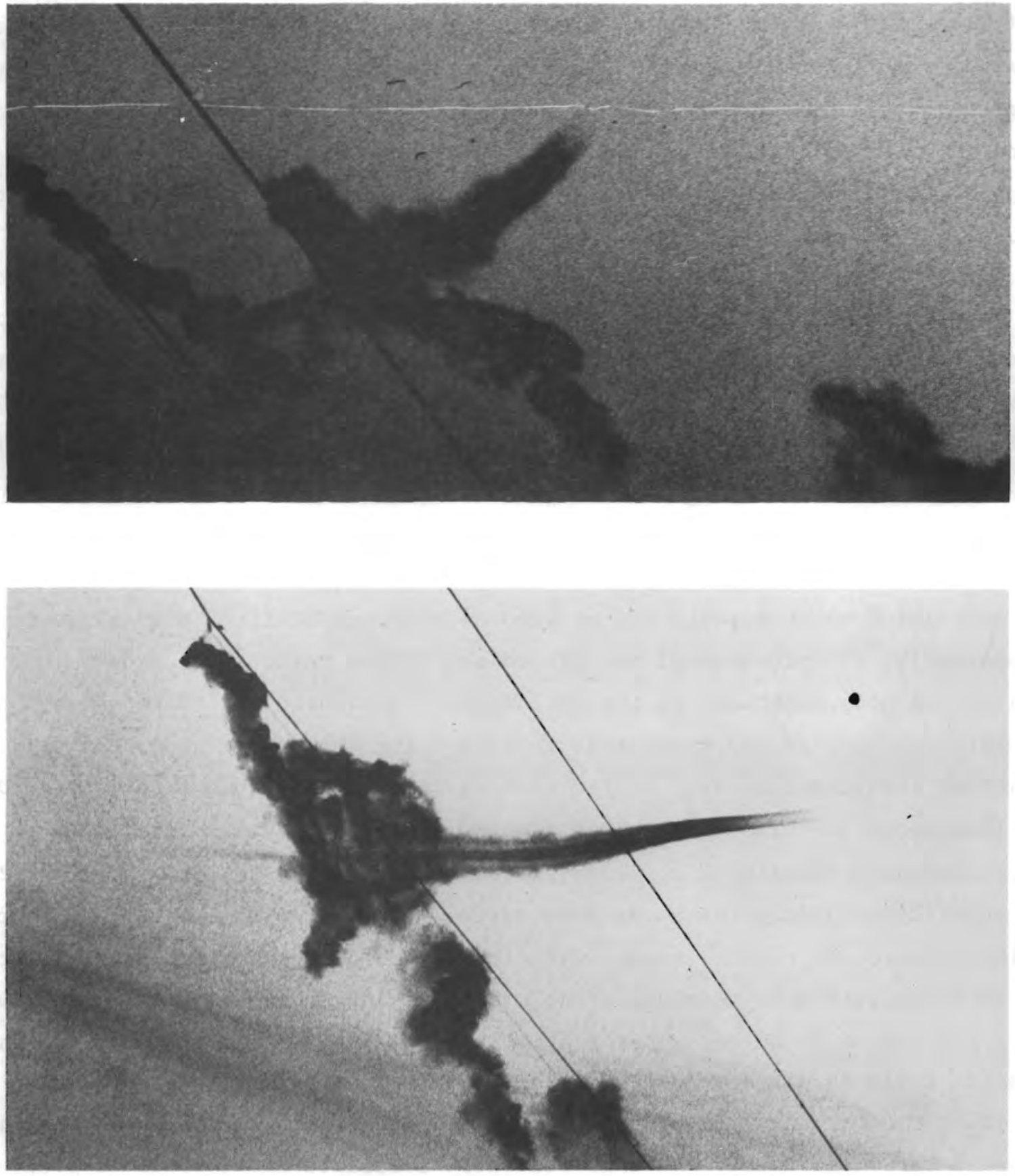

Figure 6.24 Two Spouts in the Transition State from the Forward to the Reverse Configuration 
Figure 6.24 shows two spouts in the transition state from the forward to the reverse configuration. A core (reverse spout) within a core (forward spout) is clearly observed in the two pictures. The reverse spouts are subsequently stretched and shot outward and up at a maximum speed of about $50 \mathrm{~m} / \mathrm{s}$, as estimated from Figures 6.19 and 6.20. From the same figures, two of the reverse spouts move a distance as 1 ong as about $100 \mathrm{~m}$ from the visible portion of the trails. At the later stage of the movement, the extremely long spouts do not appear to follow the helical trajectories of the vortex tubes but rather to move away from the helices.

The phenomenon of vortex core reversal, which is believed to be caused by a form of vortex instability, is not well understood. Note that the abrupt reversal is not inherent to the wake flow. A similar type of flow reversal has been observed in the trailing vortices of a $\mathrm{C}-47$ aircraft flying at about $12 \mathrm{~m}$ above the ground (Huffaker et a1. 1971) and in the wake of a MOD-0 wind turbine, (a) but neither is as dramatic as we observed. We conducted another run on the opposite side of the wind turbine with the smoke pot placed at about the same relative position referenced to the turbine. We did observe the 1 ong spouts which shoot outward and up with no evidence of flow reversal in sight. Apparently, the presence of the ground and of the turbulent boundary layer, which are nonsymetrical to the cylindrical coordinate in reference to the rotor, may play an important role in triggering the vortex core reversal. Further investigation will be required to confirm the above hypothesis. Once a long spout is formed, the smoke trapped in the original vortex tends to lose its coherency rapidly, compared with others without the spout. Figure 6.20 demonstrates clearly the above observation. For example, the vortex trains with at most one reverse spout (the first three pictures) are more persistent than those with more than one spout (the last three pictures). In other words, the generation of a long spout destabilizes the tip vortices and causes the vortex train to disperse more rapidly. For the extremely long spout that escapes the turbine wake, a part of the kinetic energy in the spout will have leaked out of the wake, which is otherwise available for turbulence generation as the vortices break up at downind stations. [f many long spouts are.

(a) Observed from two movie films on tuffs and smoke tests, provided by T. R. Richards, NASA Lewis Research Center, Cleveland, Ohio, 44135. 
generated, an appreciable amount of energy may be leaked out of the wake and the turbulence level in the wake may be significantly lower than we anticipate. The direct consequences are a slower growth of the wake height and width and a slower recovery of the momentum deficit. Increased turbine spacing would be required to overcome these problems. Therefore, the frequency of occurrence of the long spouts is an important parameter to be measured for determination of the minimum spacing between turbines.

In a review article of vortex breakdown (Leibovich 1978), the term vortex breakdown is used for a disturbance characterized by the formation of an internal stagnation point on the vortex axis, followed by reversed flow in a region of limited axial extent. At a tip speed of $83.8 \mathrm{~m} / \mathrm{s}$ for the MOD-2 turbine, a very rapid swirling flow develops near the cores of the tip vortices. Under extreme conditions, the rapidly swirling flows may lead to columnar flow reversal as observed in devices like cyclone chambers. According to Leibovich (1978), reversed axial flows of a nearly columnar nature are common in theory. Solutions to the axially symmetric Navier-Stokes equation have been derived to investigate a few classes of the above flow phenomenon (e.g., Donaldson and Sullivan 1960). However, whether the vortex core reversal can be directly related to the columnar flow reversal is yet to be determined. (a)

Another interesting phenomenon is the merging of vortices as they are being advected downwind. The evolution of the merging may be observed from the third through the sixth pictures in Figure 6.19 (more so in the movie record (Liu et al. 1982)]. In the third picture, the two upwind vortices beg in to approach each other. Complete overlap takes place after one time step, as shown in the fourth picture. In the next two time steps, the two overlapped vortices merge further and begin to form a single entity. In the movie record, merging of more than two vortices is often observed. An important consequence of the vortex merging is the possible enhancement of the vortex motion at downwind stations.

\subsubsection{Visual Results from Rocket Smoke Trails}

As shown in Figure 6.1, the presence of the rotor tip vortices can also be detected from the rocket smoke trail if it pierces through one of the helical

(a)Private communication with Professor Sidney Leibovich, Cornell University, Ithaca, New York, 1982. 
vortex trajectories. In that figure, two cusps begin to develop initially near the top of the wake. They become more distinctive with time. The top cusp evolves clearly into a vortex formation similar to those displayed by the smoke tracers released from the tether. The lower cusp does not show any trace of vortex motion at first but then evolves into a very weak vortex near its top after about 14 seconds. Apparently, the lower cusp is a part of the edge of the wake that displays a strong disk-1ike velocity deficit. The inner weak vortex observed at the top of the lower cusp appears to be generated at the junction between the controllable tip and the fixed blade where the continuity of the blade is disrupted. Incidentally, the separation between the two vortices is about the same as the length of the controllable tip ( $13.7 \mathrm{~m})$, which gives support to the above argument. From the traces of the smoke trails, it is certain that the tip vortex occupies the very top edge of the turbine wake.

The tip vortex stays intact and has no sign of breaking up for at least 13 seconds of elapse time, Figure 6.25 shows another series from a smoke trail that coincidently pierces through one of the helical vortex trajectories [RT68]. The rocket was launched at $1 D$ and $275^{\circ}$ and the wind speed and direction were $8.2 \mathrm{~m} / \mathrm{s}$ and $274^{\circ}$. Again, the tip vortex occupies the very top edge of the wake. The portion of the trail above the vortex appears to be more coherent and persistent than that below the vortex, a demarcation of the wake edge. The tip vortex observed in the figure remains intact for at least 7 seconds (the last picture taken). The entire trail, except the lower portion, is discernable for about the same amount of time, However, the smoke trail is definitely not as long lasting as that released at $1 / 2 \mathrm{D}$ (Figure 6.1), indicating a somewhat higher level of turbulence in the wake at $1 \mathrm{D}$ than at $1 / 2 \mathrm{D}$. We may generalize the finding that the tip vortex occupies the top edge of the turbine wake. From our observations in Figures 6.1 and 6.25 , we may claim that the helical vortex trajectories represent the outer edge of the turbine wake, at least initially. We do not envision any significant change in this configuration for some distance downwind before the vortex begins to break up. Eventually, the tip vortex will break up into patches of turbulence as a result of vorticity diffusion (Maxworthy 1972) and/or vortex breakdown (Leibovich 1978, Hall 1972). In the presence of turbulence, the diffusion rate and vortex instability enhance accordingly, resulting in a faster breakup of the $t i p$ 

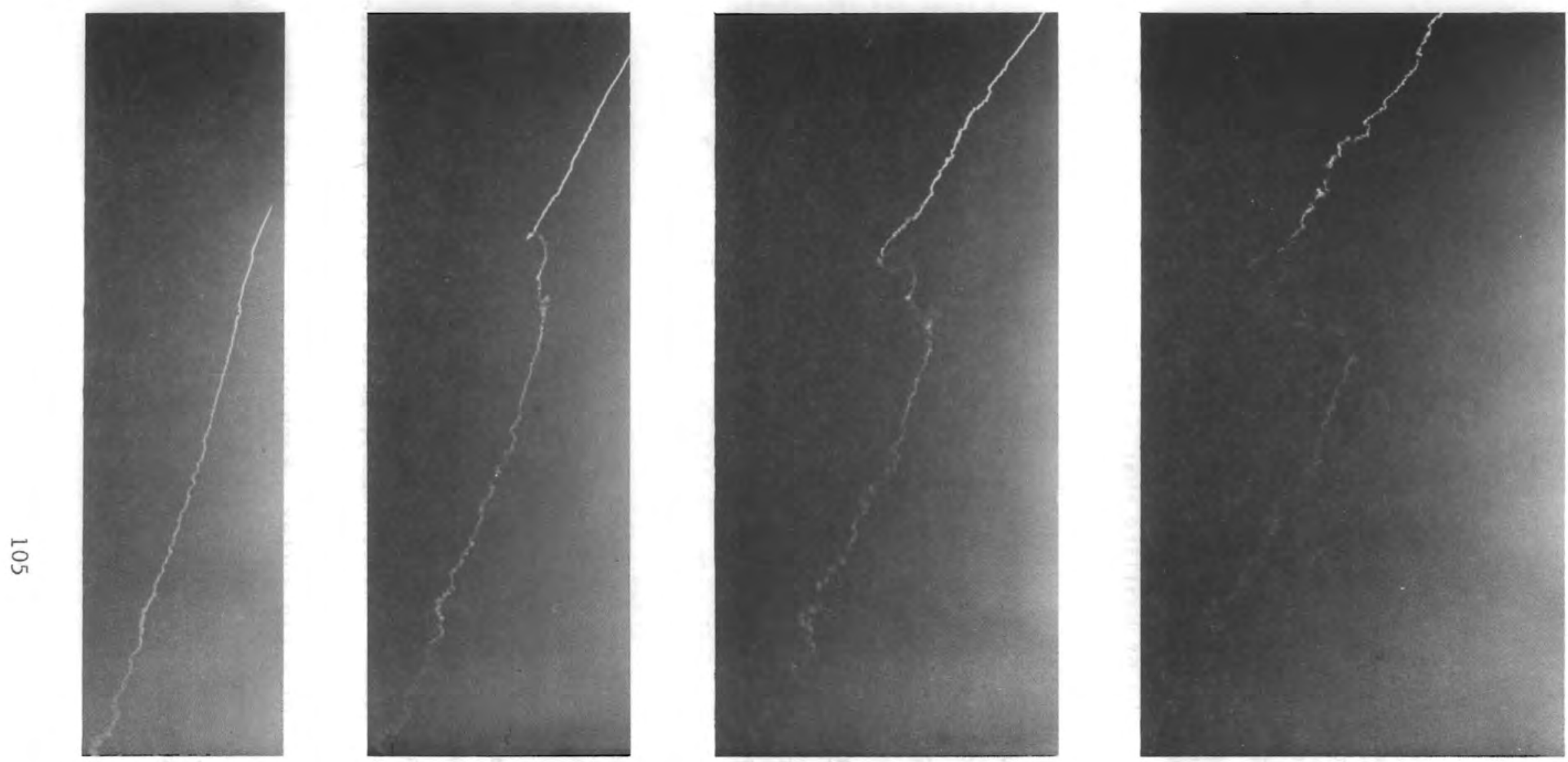

WIND DIRECTION

Figure 6.25 Sequential Pictures of a Single Smoke Trail at 1D and $\phi=275^{\circ}$ [RT68] .

The pictures were taken looking south, and the time between pictures is 2 seconds. The evolution of the tip vortex near the top of the wake is demonstrated with clarity. 
vortex. As mentioned in this section, the generation of long spouts, which results from a new form of vortex instability, tends to hasten the breakup of the tip vortex. Therefore, the tip vortex that is very coherent and organized upon generation is a source of wake turbulence at downind stations. As discussed in Section 6.1, the turbulence intensity appears to increase with increasing downwind distances. We anticipate that the breakup of the tip vortex contributes significantly to the increase of the turbulence intensity at downwind stations. It should be noted that we were unable to identify any tip vortices in all the smoke trails released at $2 \mathrm{D}$ chrough $6 \mathrm{D}$. Of course, we have to bear in mind that the probability that a trail will pierce one of the helices is very low. The fact that some vortices or a portion of the helices becone less coherent or unstable (or even break up into turbulent patches) further reduces the above probability. Other methods were used in an attempt to determine the persistence of the tip vortex. One of these methods is the use of flow streamers to detect the presence of tip vortices.

\subsubsection{Visual Results from Flow Streamers}

Several streamer runs were conducted to observe the presence of tip vortices at distance from 1/2D to about 4D. Two types of streamers were attached to the tethered line of a parafoil kite, as illustrated in Figure 5.7. The first type was $11-\mathrm{m}-1$ long crepe paper ( $5-\mathrm{cm}$ wide) and the other was 12-m-1ons fabric strips ( $2-c m$ wide). The crepe paper had a much better response to the flow than did the fabric strips, but the former also tended to be torn apart easily, especially when wet. We ended up using both fabric strips at 1/2D, $2 \mathrm{D}$, and $4 \mathrm{D}$ and crepe paper at $2 \mathrm{D}$. At about $1 / 2 \mathrm{D}$, the streamers responsed to the vortex motion very well. Figure 6.26 shows four series of streamer patterns at about 1/2D. The pictures were taken sequentially from top to bottom at a speed of 3.5 frames/second. Unfortunacely, it is very difficult to relate from still pictures the organized, three-dimensional motion of the streamers in response to the passage of a tip vortex. Each cycle of the motion lasts for a second or so. We could only take three to five pictures within a cycle for the film speed used. From the movie records (Liu et al. 1982), however, we are able to correlate better the vortex and the streamer motions. Whenever a streamer intercepts a tip vortex, the motion of the streamer in response to 

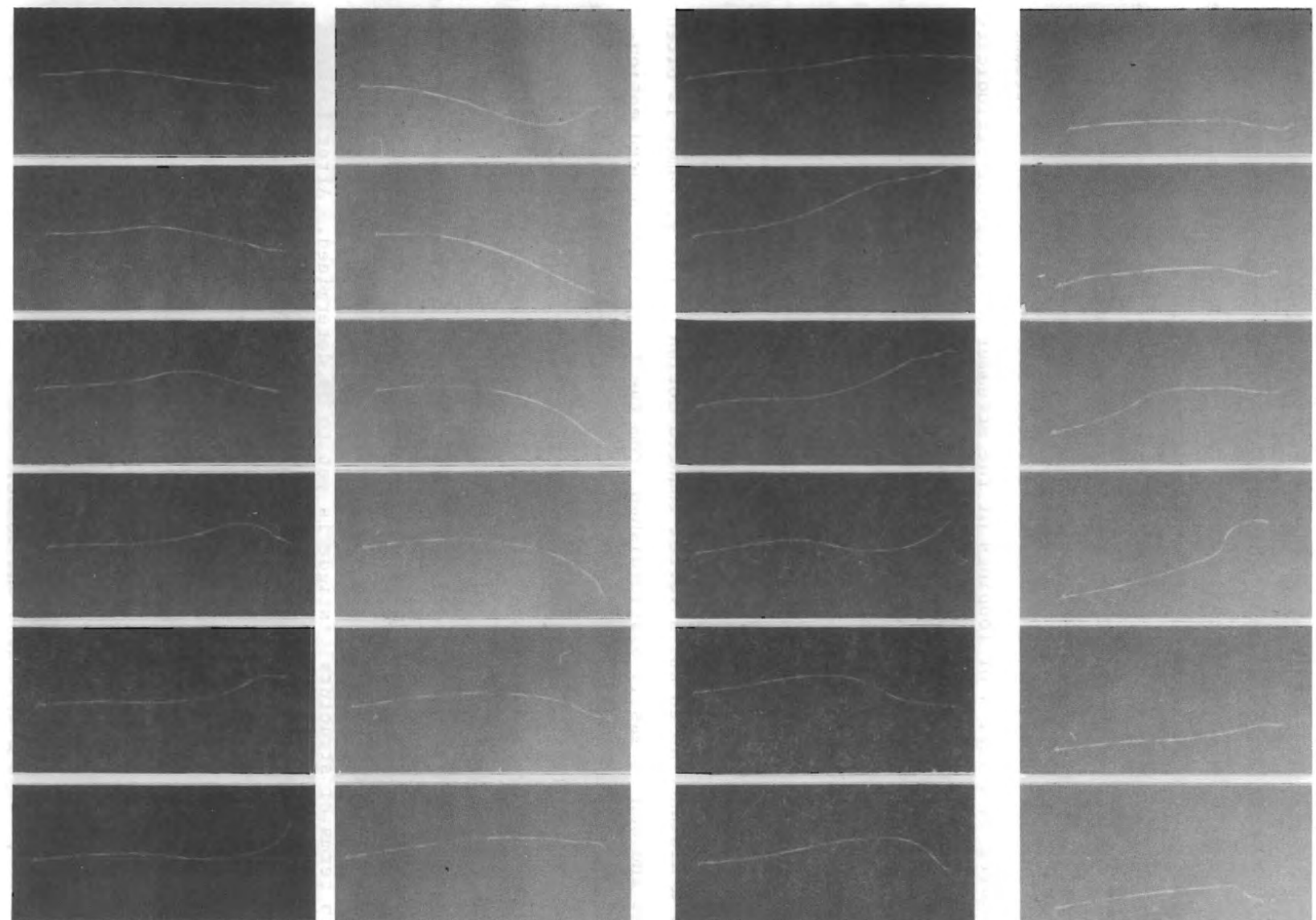

Figure 6.26 Four Series of Pictures of the Motion of Streamers Caught in the Tip Vortices [FS73]. The streamers are about $1 / 2 \mathrm{D}$ downwind of the rotor on the southern edge of the wake. 
the vortex motion is characterized by a "looping" of the entire streamer. The looping has a definite direction corresponding to the sense of the vortex motion. It also has a reasonably regular rhythm. Such characteristics of the looping motion can be easily distinguished from the random motion induced by large turbulent eddies in the wake. We deployed the kite with streamers three to four times during the field experiments, and we made many observations to gain sufficient experience for identifying the characteristics of the looping motion. On many occasions, a train of vortices catches a particular streamer that results in a series of loopings of the streamer with regular periodicity. On other occasions, more than one streaner was caught by passage of a tip vortex, which results in a sequence of loopings of the streamers involved. The periodicity of motion involving more than one streamer makes it even easier to distinguish the vortex and turbulent motions in the wake. The following description in terms of the vortex persistence is derived from many observations of the streamer runs, two of which are including in the formal movie record by Liu et al. (1982).

For a distance up to $2 \mathrm{D}$, a vortex-induced motion of the streamer is often observed and can be easily distinguished from the turbulence-induced motion. At a distance of $4 \mathrm{D}$ and beyond, the vortex-induced motion is very weak and can no longer be easily distinguished from the turbulence-induced motion. The intensity of the turbulence-induced motion is reduced noticeably when the kite is moved out of the wake. Based on our observations, we are confident that the tip vortex will have an insignificant "catastrophic" impact on the structure of downwind turbines with separations greater than or equal to 4D. Whether the same can be said of the long-term impact as compared with that of wake turbulence in terms of structural fatigue is yet to be determined. After losing some of its coherence or even undergoing partial breakup, the tip vortex is still more periodic than the wake turbulence. We anticipate a certain degree of energy concentration at the harmonics of the rotational frequency of the turbine, as attributed to the presence of the tip vortex or its derivatives in the wake. Further research is required to deterine whether such energy concentration is significant as compared to the spectral density of wake turbulence. Note that part of the wake turbulence is attributed to the breakup of tip vortices, which complicates the issue. 


\section{REFERENCES}

Abramovich, G. N. 1963. The Theory of Turbulent Jets. Massachusetts Institute of Technology Press, Cambridge.

Ainslie, J. F. 1981. Computer Modelling of Wake Effects in Clusters of Wind Turbines. Central Electricity Generating Board Memorandum LM/ PHYS/229, Leatherhead, U. K.

Alfredsson, P. H. and J. O. Dahlberg. 1979. A Preliminary Wind Tunnel Study of Windmill Wake Dispersion in Various Flow Conditions. Technical Note FFA-AV-1499, Part 7, The Aeronautical Research Institute of Sweden, Stockholm.

Axell, R. A. and H. B. Woody. 1981. "Test Status and Experience With the 7.5 Megawatt MOD-2 Wind Turbine Cluster." In Large Horizontal Axis Wind Turbines. CONF-810752, National Technical Information Service, Springfield, Virginia.

Boschloo, G. 1977. Wake Structure of a Darrieus Rotor. TNO Report 77-07244, Organization for Industrial Research (TNO), Apeldoorn, The Netherlands.

Builtjes, P. J. H. 1979. Wind Turbine Wake Effects. TNO Report 79-08375, Organization for Industrial Research (TNO), Apeldoorn, The Netherlands.

Builtjes, P. J. H. and D. J. Milborrow. 1980. "Modelling of Wind Turbine Arrays." In Proceedings of the Third International Symposium on Wind Energy Systems, PP. 417-430, Copenhagen, Denmark (August 26-29, 1980). BHRA Fluid Engineering, Cranfield, Bedford, England.

Crafoord, C. 1979. Interaction in Limited Arrays of Windmills. Report DM-26, Department of Meteorology, University of Stockholm.

Donaldson, C. duP. and R. D. Sullivan. 1960. "Behavior of Solutions of the Navier-Stokes Equations for a Complete Class of Three-Dimensional Viscous Vortices." In Proceedings of the 1969 Heat Transfer Fluid Dynamics Institute, $\mathrm{pP} \cdot 16-30$, Stanford University Press.

Durst, F., B. M. Howe and G. Richter. 1982. "Laser-Doppler Measurement of Crosswind Velocity." Appl. Optics 21:2596-2607.

Faxen, T., A. S. Smedman-Hogstrom and V. Hogstrom. 1978. The Meteorological Field Project at the Wind Energy Test Site, Kalkugnen, Sweden. Report No. 51, Department of Meteorology, University of Uppsala.

Ha 11, M. G. 1972. "Vortex Breakdown." Ann. Rev. Fluid Mech. 4:195-218.

Hansen, A. C. 1981. "Measurement of the Wake of an Aeropower SL1000 Wind Turbine." In Proceedings, Volume II, Fifth Biennial Wind Energy Conference and Workshop, pP. 347-360. SERI/CP-635-1340, Nationa1 Technical Information Service, Springfield, Virginia. 
Hiester, T. R. and W. T. Pennell. 1981. The Meteorological Aspects of Siting Large Wind Turbines. PNL-2522, Pacific Northwest Laboratory, Richland, Washington.

Huffaker, R. M., A. V. Jelalian, W. H. Keene and C. M. Sonnenschein. 1971. "Application of Laser Doppler Systems to Vortex Measurement and Detection." In Proceedings of a Symposium on Aircraft Wake Turbulence, pp. 113-124, Plenum Press, New York.

Leibovich, S. 1978. "The Structure of Vortex Breakdown." Ann. Rev. Fluid Mech. 10:221-246. 
APPENDIX A

ROCKET DESIGN AND ASSEMBLY 


\section{REFERENCES (cont.)}

Thwaites, B., ed. 1960. Incompressible Aerodynamics. Clarendon Press, Oxford.

Vermeulen, P. E. J. 1978. A Wind Tunnel Study of the Wake of a Horizontal Axis Wind Turbine. TNO Report 78-09674, Organization for Industrial Research (TNO), Apeldoorn, The Netherlands.

Vermeulen, P. E. J. 1980. "An Experimental Analys is of Wind Turbine Wakes." In Proceedings of the Third International Symposium on Wind Energy Systems, pP. 431-450, Copenhagen, Denmark (August 26-29, 1980). BHRA Fluid

Engineering, Cranfield, Bedford, England.

Vermeulen, P.E. J. and P. J. H. Builtjes. 1981. Mathemetical Modelling of Wake Interaction in Wind Turbine Arrays. TNO Report 81-01473, Organization for Industrial Research (TNO), Apeldoorn, The Netherlands.

Vermeulen, P. E. J., P. J. H. Builtjes, J. Dekker and G. L. V. Bueren. 1979. An Experimental Study of the Wake Behind a Full Scale Vertical Axis Wind Turbine. TNO Report 79-06118, Organization for Industrial Research (TNO), Apeldoorn, The Netherlands. 


\section{APPENDIX A}

\section{ROCKET DESIGN AND ASSEMBLY}

The rocket used for our flow visualization experiments is made up basically of two halves. The bottom half holds the motor and fins and also provides a cavity to contain the recovery system. The top half supports the smoke deployment system and nose cone.

The bottom half or "motor tube" is made of 3-cm-ID plastic-impregnated cardboard tube. This tubing is readily available from local hobby shops but, because we needed a quantity, we ordered ours direct from F1ight systems Incorporated (FSI), Raytown, Missouri. A thrust ring is attached inside the tube $10.2 \mathrm{~cm}$ from the bottom to keep the motor from thrusting up through the tube. This ring is made of phenolic so that the heat from the engine does not incinerate it. Also inside but halfway up the tube is attached a 0.6-m length of $\# 24$ gauge wire that acts as a shock chord to keep the two halves of the rocket together after the deployment charge goes off. Three $1.6-\mathrm{cm}$ birch plywood fins are epoxied on the outside of the motor tube at the very bottom. The entire motor tube is painted with aluminum paint for flame resistance.

The top half of the rocket is made of a $17.8-\mathrm{cm}$ length of $1.1-\mathrm{cm}$ hardwood doweling with two $2.8-\mathrm{cm}-\mathrm{d}$ ia collars spaced $12.7 \mathrm{~cm}$ apart. This creates a 12.7-cm spool with a 5.08-cm-long nipple projecting from one end. A 25.4-cm piece of 1.27-cm-OD, $0.05-\mathrm{cm}-w a 11$ aluminum tubing is epoxied onto this nipple. At the other end of this tube is a disk made of $1.27-\mathrm{cm}$ honeycomb and graphite that is cut to size to fit the nose cone (which was actually the pointed half of a Leggs pantyhose container). The top collar on the spool has a shoulder around its upper half so that when the spool is inserted within the motor tube, the shoulder acts as a stop, thus resulting in a rocket that is $73.7 \mathrm{~cm}$ in overall length with a weight of $15 \mathrm{oz}$ and that breaks apart in the middle to facilitate parachute deployment. The parachute is an eight-sided chute made of 4-oz ripstop nylon with heavy carpet thread for shrouds. It is attached to an eye screw in the hardwood dowel between the two collars with a $25.4-\mathrm{cm}$ piece

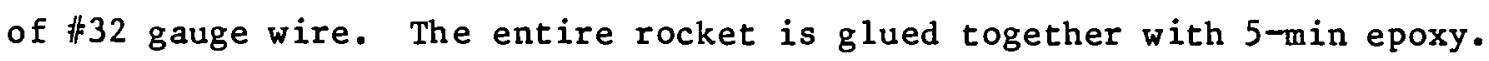

The smoke trail is created by four orange smoke bombs. These are simply wired onto the $1.27-\mathrm{cm}$ aluminum tube directly below the graphite disk with 
regular bailing wire. The smoke bombs were obtained from $\mathrm{E}$. Vernon Hill, Inc. They have a 1-min burn time and produce approximately $22.7 \mathrm{~m}^{3}$ of smoke each.

The booster used is an E60-4, which can only be obtained from FSI in Missouri. This booster has a total impulse of $30 \mathrm{~N} / \mathrm{s}$ with a maximum thrust of $88.9 \mathrm{~N}$. It has a $0.6-\mathrm{s}$ thrust time and a $4-\mathrm{s}$ charge. It is mounted in the rocket by wrapping masking tape around it until it can be forced into the tube with approximately $4.5 \mathrm{~N}$ of force. This ensures that the motor does not dislodge when the ejection charge goes off.

To prepare the rocket for launch, the parachute is packed around the spool on the nose piece and inserted into the motor tube. An aluminum foil shroud is placed around the joint for stream 1 ining and to protect the chute from the heat of the smoke bombs. The smoke bombs are then wrapped in tin foil so the cardboard casings will not ignite and wired onto the aluminum tubing in a cluster of four immediately against the composite disk at the top of the rocket. Finally, a flameproof wadding is packed into the bottom of the motor and the motor is fitted as described earlier.

The launch pads are of the hollow tower type and are made from an aluminum " $T$ " section bolted to an aluminum base plate. Two adjustable legs are attached on one side to adjust the tilt of the pad. This capability is required because a properly trimmed rocket will turn into the wind. Therefore, the launch pad is tilted slightly downwind to end up with a verticle flight path. The operator at the launch pad is in radio contact with the camera operators to inform them when a smoke bomb is 1 it; they also receive a 10-s countdown to launch so that the cameras can be started at the proper time to view the run. 
APPENDIX B

IMPLEMENTATION OF THE TIP VORTEX VISUALIZATION 
As stated in the proposal, no unusual equipment was necessary for this task. The object was to suspend a smoke source close to but behind the tip of the turbine blade while the turbine was in operation.

A system was devised using three ordinary $5.1-\mathrm{cm}$ pulleys and a 213.4-m length of $0.6 \mathrm{-cm}$ polypropylene rope to suspend the smoke pot. Climbing carabiners were used to attach two of the pulleys approximately $3 \mathrm{~m}$ apart to the floor grating of the catwalk at the 57.9-m level of the turbine tower. On the ground, another pulley was attached to a length of rope. Then the 213.4-m rope was threaded through all three pulleys and tied into a loop. When the bottom pulley was stretched out, a continuous loop of rope extended from the catwalk to the ground. A small pulley was tied onto one leg of this loop with a $0.3-\mathrm{cm}$ nylon line strung through it. In this way we obtained two-dimensional resolution of the entire area below the loop. We could either move the sma 11 pulley in and out or the smoke pot up and down on the $0.3-\mathrm{cm}$ line to find the optimum location for visualizing the tip vortices. Both tethers were graduated so that after a run we could determine the location of the smoke source.

The tether was anchored directly behind the turbine when not in use. During a test, the bottom anchor was attached around a person's waist and the tether was wrapped around until it was approximately parallel with the blades. Next, the small pulley was positioned so that it was approximately $6.1 \mathrm{~m}$ inside the blade tip. Then, a smoke pot was attached to one end of the $0.3-\mathrm{cm}$ nylon rope with a wire hanger, which kept the hot smoke pot from burning through the rope tether. After all was set, the smoke pot was ignited and raised into position. We could then note the position of the smoke pot with respect to the tip vortices and, hence, get an approximate location of the tip vortices. 

APPENDIX C

COMPUTER ANALYSIS 
A program named DEFICIT was developed to read the digitizer data files, correct the data for lens distortion, create a plottable data file of the corrected position data, and to calculate the velocity profile. A listing of the program is included at the end of this appendix; sample printouts of both the program inputs and outputs are self-explanatory.

The program requires an input data file having a typical sequential format as follows:

$$
\left.\begin{array}{l}
\phi, \phi, 4 \\
6, \text { XREF, YREF } \\
\phi, x, y \\
\phi, x, y \\
\phi, x, y \\
10, x R E F, \text { YREF } \\
\phi, x, y \\
\phi, x, y \\
\phi, x, y
\end{array}\right\}
$$

Frame Step

1st digitized track

2nd digitized track

The structure of the file can be repeated for as many rocket trails as are valuable to digitize. The first line in the file contains the frame step, which indicates the number of slides that are changed between each digitization. For example, if the motor drive was set at $4 \mathrm{frames} / \mathrm{s}$, and a frame is digitized every second, then the frame step is 4.

Starting with the second line, a header line is placed at the start of each rocket trail digitization designating the absolute slide number in the sequence for any single rocket launch. Then a reference point (e.g., a fence post or telephone pole) is digitized so that a frame positioning error can be corrected. Following this header line, a sequence of about 100 data points is entered, preceded by a zero, that follows the outline of the rocket trail. DEFICIT looks for a data file name having the typical form DIGI/265.4D$183 /$ RT12, where the first four letters are the file type descriptor (in this 
case raw data), the next three digits the rocket baseline orientation, then the distance down axis (4D). The number 183 is the off-axis camera distance, which is followed by the run number.

The following is a sample input to DEFICIT for a 6D rocket run (underlined data represents keyboard or command file input):

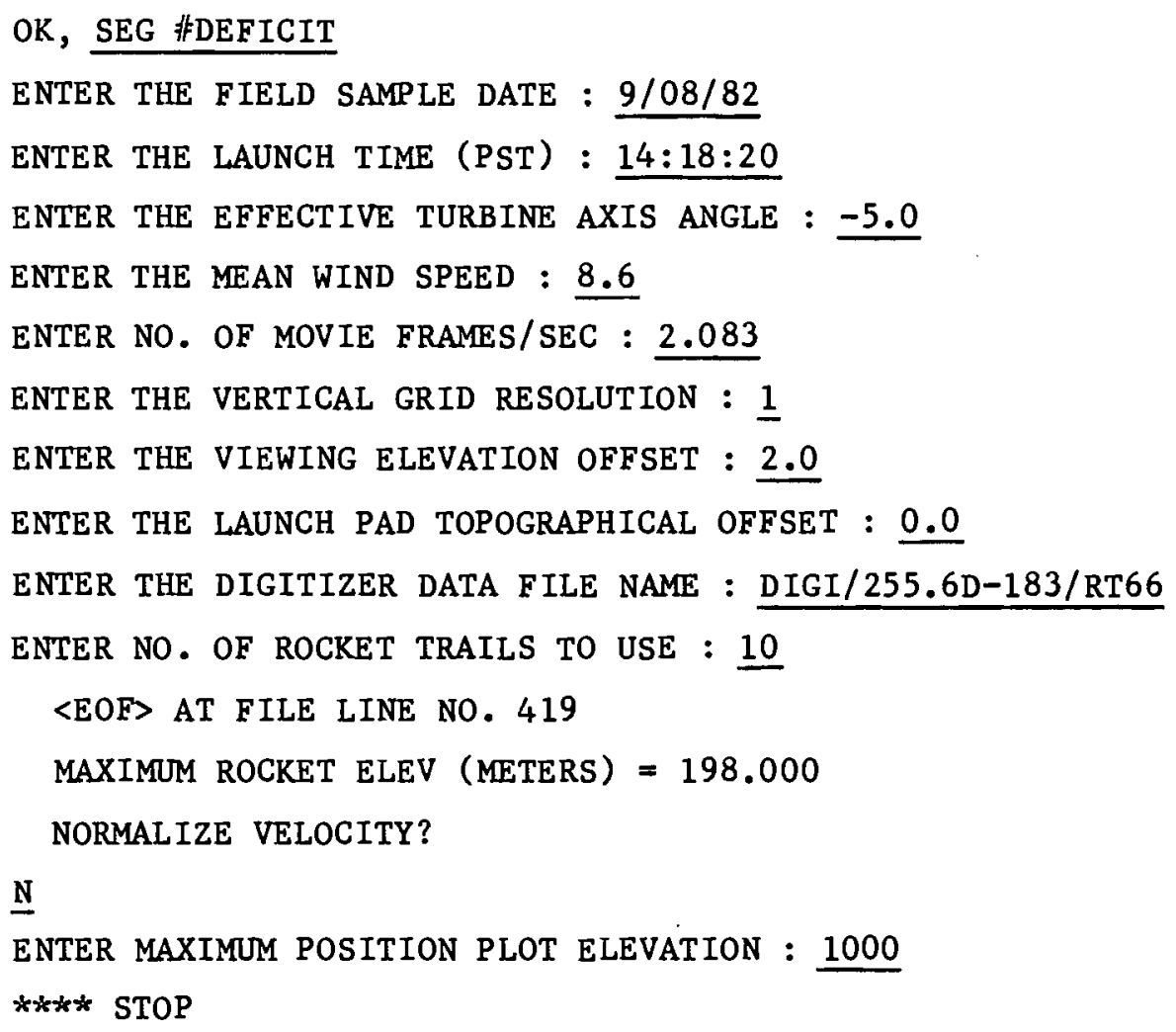

The following page shows the velocity profile output file from DEFICIT. Three files are created by DEFICIT for every input data file having the DIGI file type descriptor prefix. The velocity profile data are contained in the VELP files, the corrected position data are in the POSN files, and the rocket trajectory path coordinates are stored in the PATH files.

The computer program is attached following the velocity profile output. 
FILE : VELP/255. 6D-183/RT66

FIELD SAMPLE DATE : $9 / 08 / 82$

FIELD SAMPLE P. S. T. (HH: MM: S5) : 14:18: 20

PRDCESSING DATE : $11: 08: 23$ FRI, 17 SEP 1982

EFFECTIVE TURBINE AXIS ANGLE $=-5$.

* LAUNCH SITE POSITION IN TURBINE COORDINATES * *

DOWN RANGE DIST $=548.64$ OFF-AXIS DIST $=-47.88$

MEAN WIND SPEED $=8.60$

MEAN DIGITIZER FRAME ADVANCE TIME $=0.96$

MAXIMUM DIGITIZED ROCKET ELEVATION $=198$

VERTICAL RESOLUTION (METERS) $=1.00$

VERTICAL ELEVATION CORRECTION $=2.00$

NUMBER OF ROCKET TRAILS USED $=6$

$\begin{array}{ll}3.00 & 8.83 \\ 4.00 & 9.05 \\ 5.00 & 9.33 \\ 6.00 & 8.97 \\ 7.00 & 10.3 \\ 8.00 & 8.21 \\ 9.00 & 8.15 \\ 10.0 & 8.17 \\ 11.0 & 8.19 \\ 12.0 & 8.22 \\ 13.0 & 8.25 \\ 14.0 & 8.28 \\ 15.0 & 8.16 \\ 16.0 & 8.04 \\ 17.0 & 7.96 \\ 18.0 & 7.75 \\ 19.0 & 7.55 \\ 20.0 & 7.39 \\ 21.0 & 7.32 \\ 22.0 & 7.24 \\ 23.0 & 7.18 \\ 24.0 & 7.10 \\ 25.0 & 7.02 \\ 26.0 & 6.88 \\ 27.0 & 6.71 \\ 28.0 & 6.52 \\ 29.0 & 6.32 \\ 30.0 & 6.16 \\ 31.0 & 6.04 \\ 32.0 & 5.94 \\ 33.0 & 5.94 \\ 34.0 & 5.99 \\ 35.0 & 6.07 \\ 36.0 & 6.17 \\ 37.0 & 6.31 \\ 38.0 & 6.38 \\ 39.0 & 6.41 \\ 40.0 & 6.36 \\ 41.0 & 6.26 \\ 42.0 & 6.17\end{array}$


Compiled on: 820919 at: 13:01 by: FORTRAN-77 Rev 18.2. 1

Options: OPTIMIZE-2 NOBIG INTS LOGL DYNM UPCASE ERRTTY

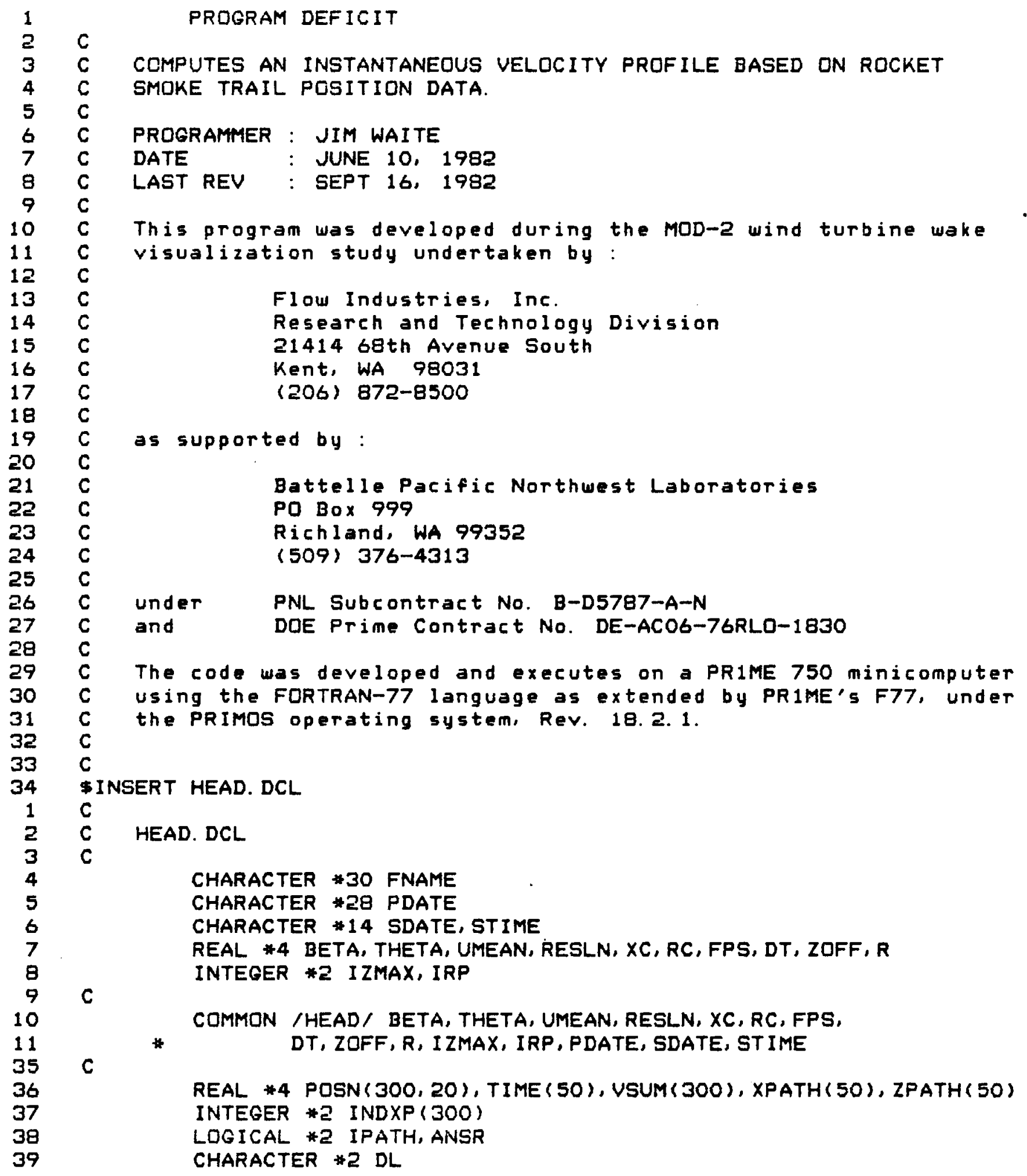


Source File: SDISK14>RTO001:WAITEPDEFICIT

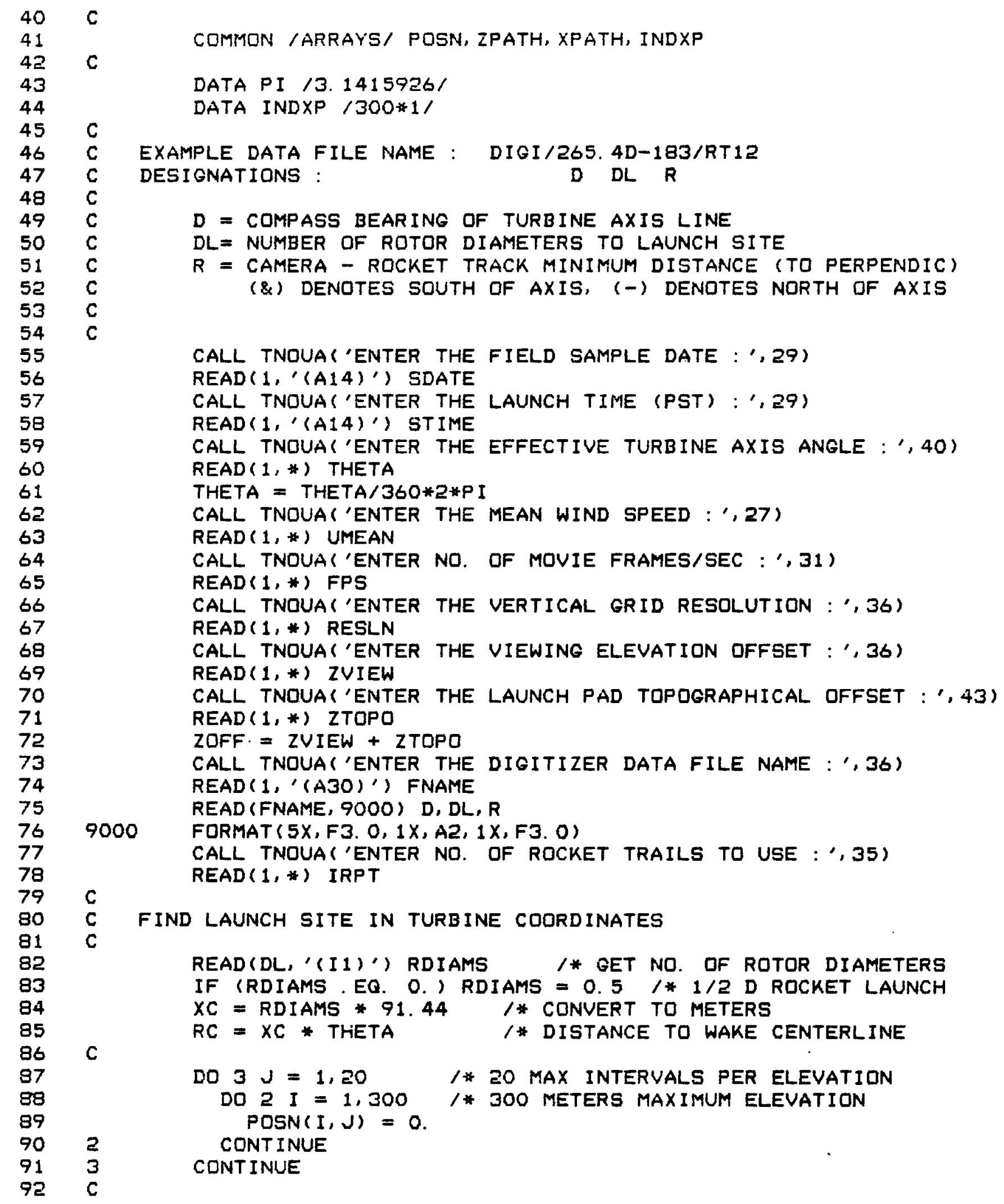




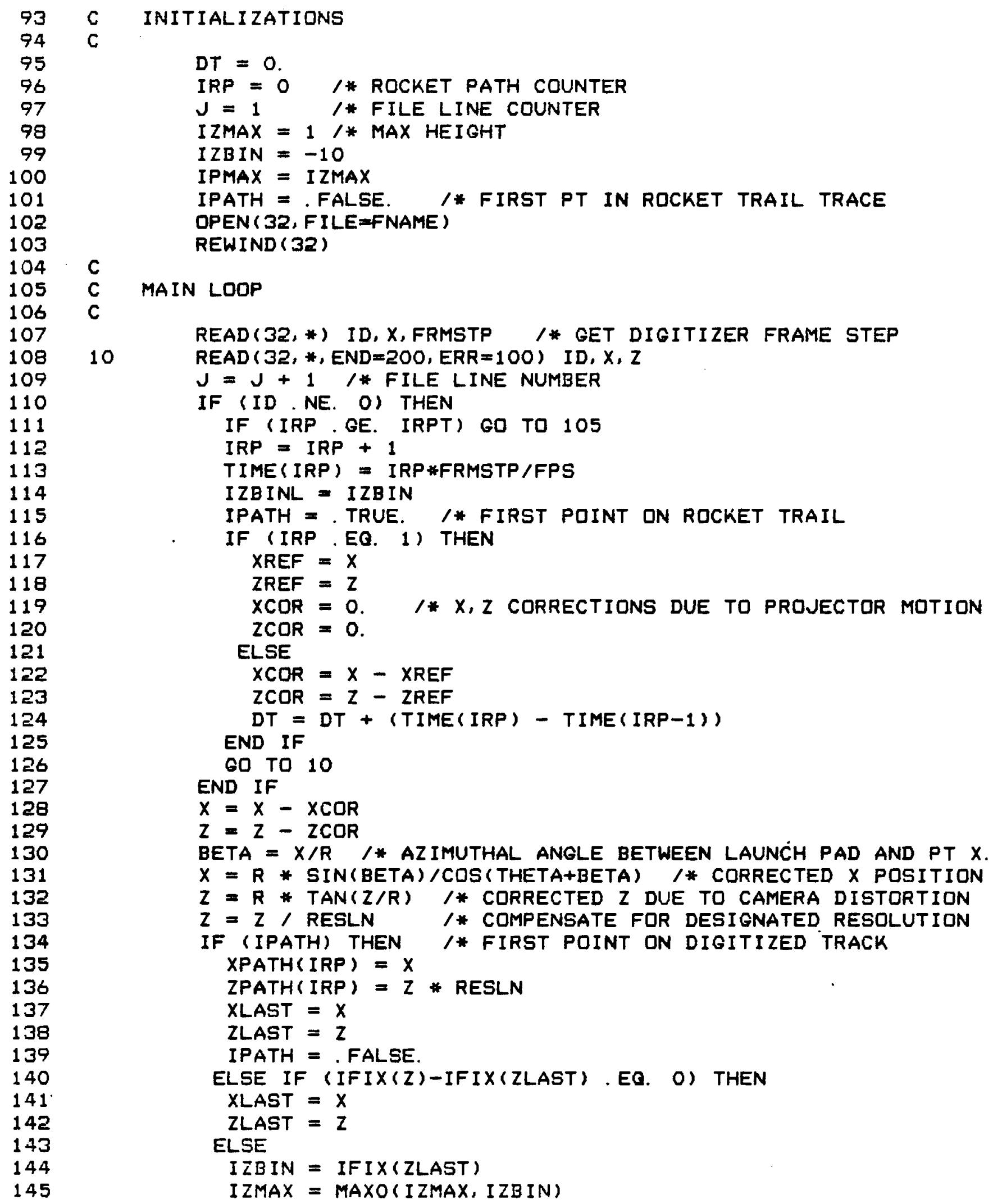




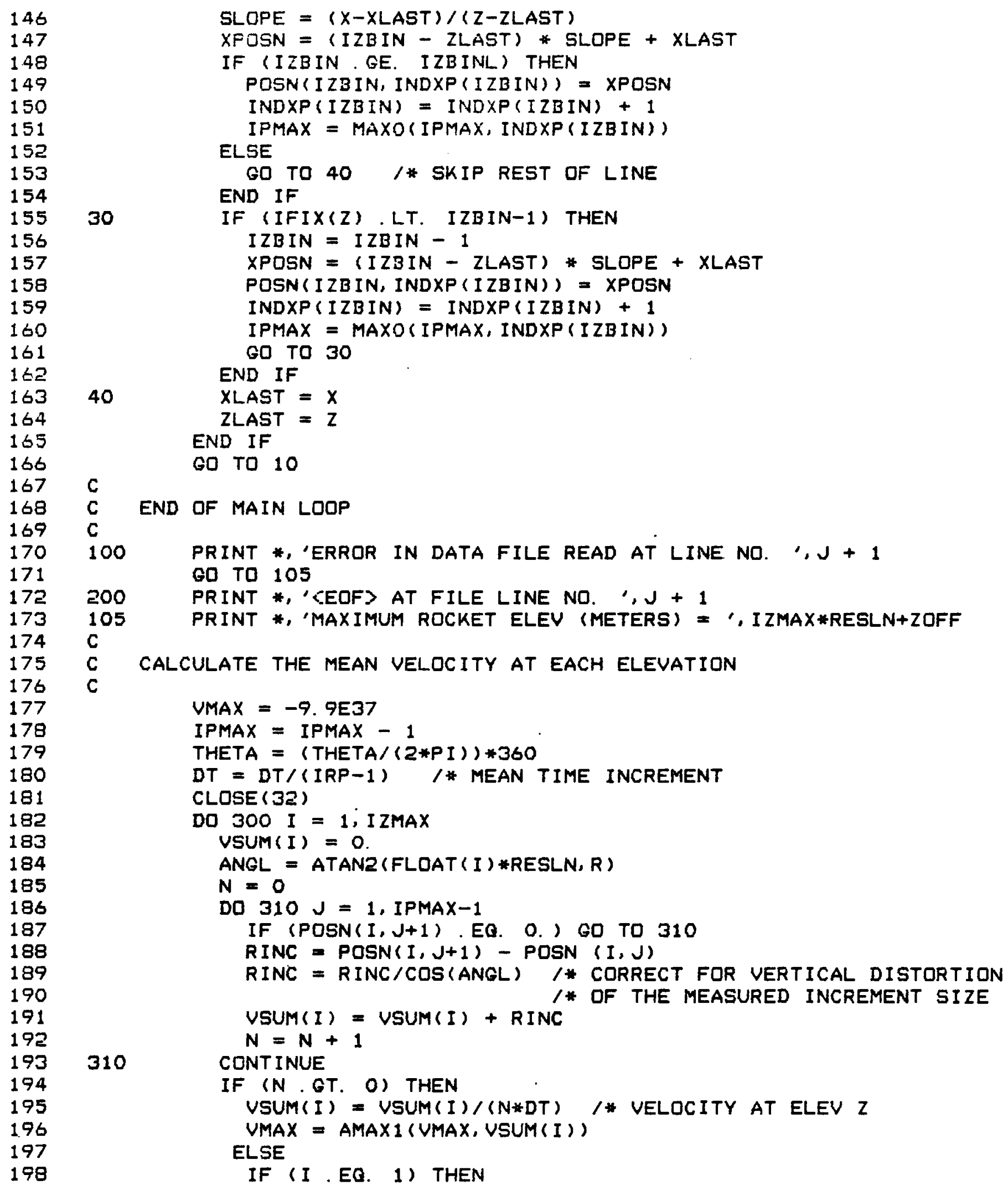

146

147

148

149

150

151

152

153

154

155

156

157

158

159

160

161

162

163

164

165

166

167

168

169

170

171

172

173

174

175

176

177

178

179

180

181

182

183

184

185

186

187

188

189

190

191

192

193

194

195

196

197

198 
199

200

$\Xi 01$

202

203

204

205

206

207

208

209

210

$\supseteq 11$

212

213

214

215

216

217

218

219

220

221

222

223

224

225

226

227

228

229

230

231

232

233

234

235

236

237

238

239

240

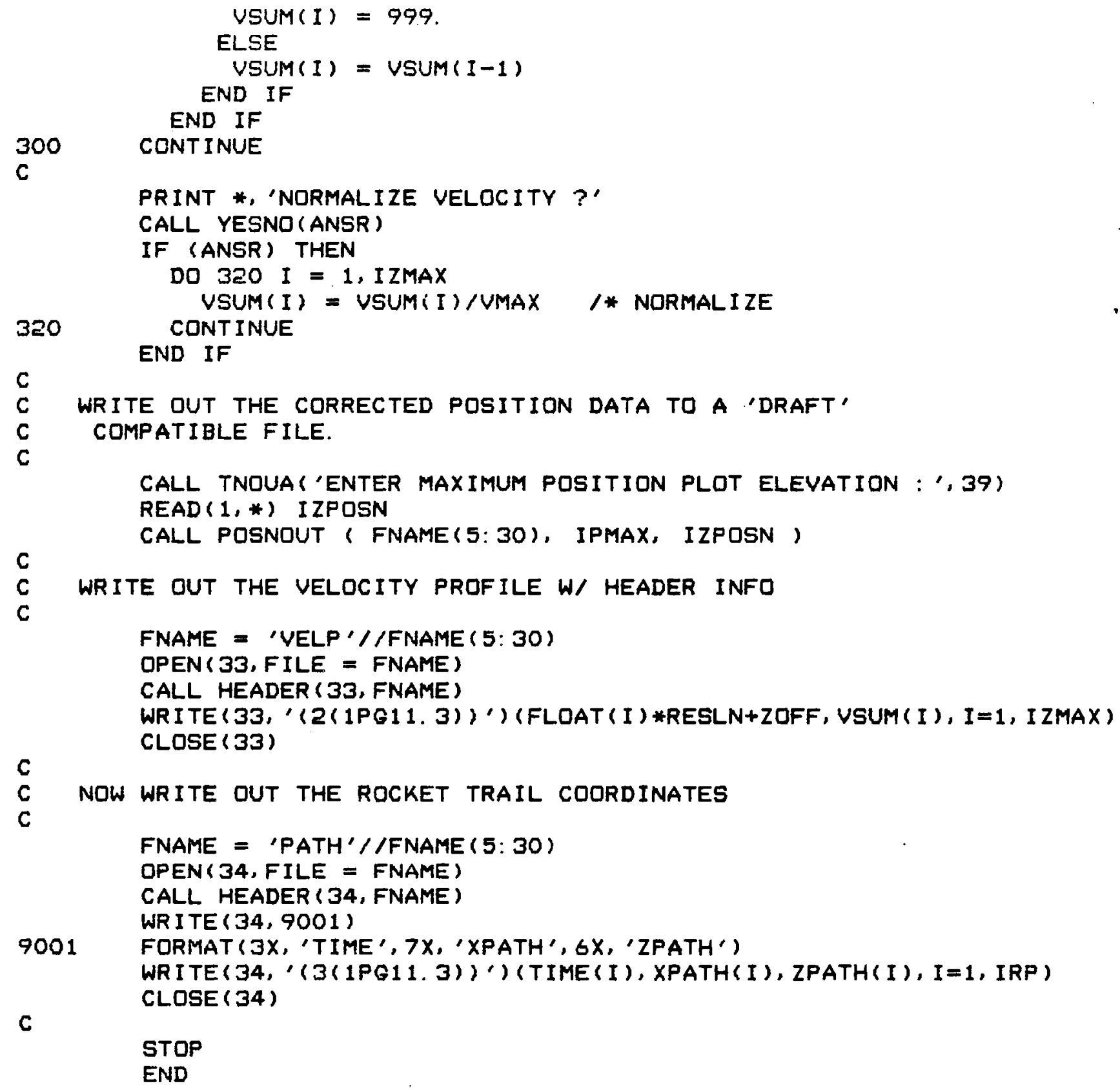




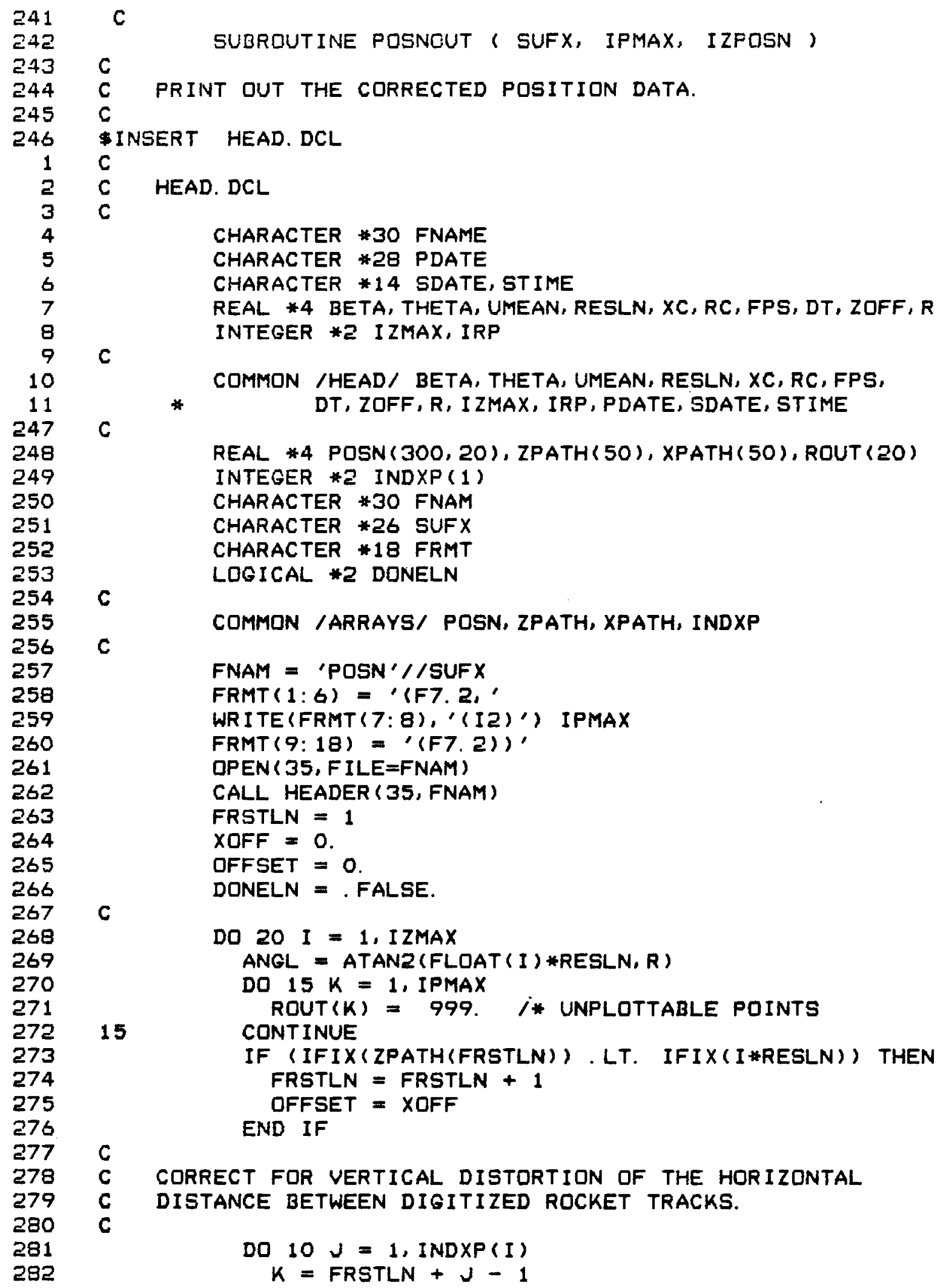


10

20

C
IF ( $(P O S N(I, J)$. EQ. O. ). QR. DONELN ) THEN ROUT(K) $=999$. DONELN $=$. TRUE.

ELSE IF ( $J$. EQ. 1) THEN

ROUT $(K)=\operatorname{POSN}(I, J)+$ DFFSET

$X F I X=R O U T(K)$

ELSE

ROUT $(K)=(\operatorname{POSN}(I, J)-\operatorname{POSN}(I, J-1)) / \operatorname{COS}(A N G L)+X F I X$ IF $(J$.EQ. 2) XOFF $=\operatorname{ROUT}(K)-\operatorname{POSN}(I, J)$ $X F I X=$ ROUT $(K)$

END IF

CONTINUE

DONELN $=$. FALSE.

IF (IZMAX . GT. IZPOSN) GO TO 20 /* DON'T PLDT HIGH ELEV. WRITE (35, FRMT) FLOAT (I)*RESLN+ZOFF, (ROUT (K), $K=1$, IPMAX) CONTINUE

CLOSE (35)

RETURN

END 


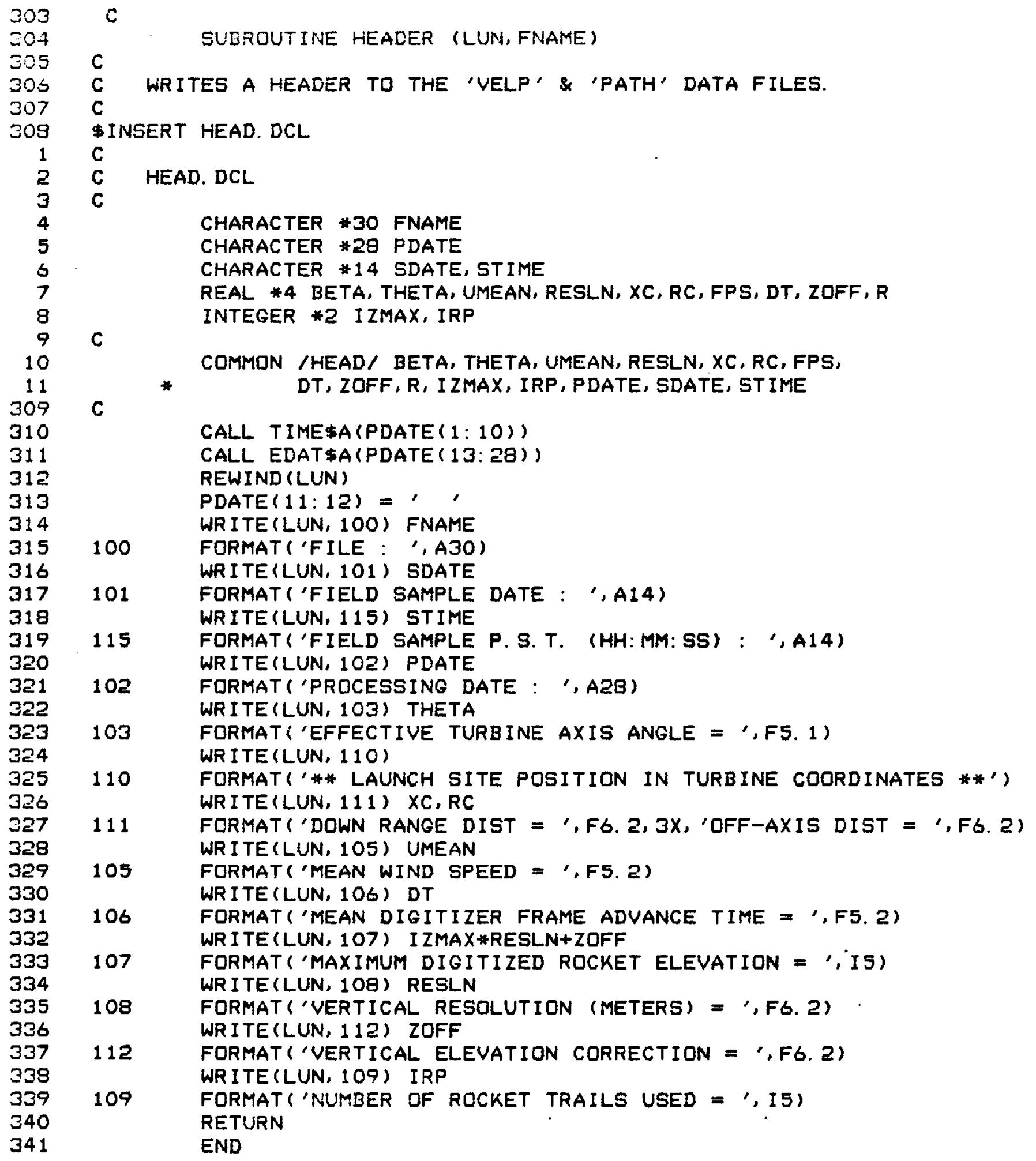




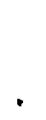




\section{DISTRIBUTION}

No. of

Copies

OFFSITE
No. of

Copies
D. Ancona

Department of Energy

Wind Energy Technology Division

1000 Independence Avenue

Forrestal Building, Room 5F059

Washington, DC 20585

C. I. Aspliden

Battelle Memorial Institute Washington Operations Office 2030 M Street N.W.

Washington, DC 20036

L. V. Divone

Department of Energy

Wind Energy Technology Division

1000 Independence Avenue

Forrestal Building, Room 5F059

Washington, DC 20585

G. P. Tennyson

Department of Energy

Albuquerque Operations Office

P.0. Box 5400

Albuquerque, NM 87110

27 DOE Technical Information Center

10 P.B.S. Lissaman

Aerovironment, Inc.

145 Vista Avenue

Pasadena, CA 91107

K. C. Spengler

American Meteorological Society

45 Beacon Street

Boston, MA 02108

T. Gray

American Wind Energy Association 2010 Massachusetts Avenue N.W. Washington, DC 20036
5 M. Bovarnick

Wind Turbine Group

Boeing Engineering \& Construction

P.0. Box 3707

Seattle, WA 98124

N. Butler

Bonneville Power Administration

P.0. Box 3621

Portland, OR 97208

2 E. J. Warchol/R. H. Holeman

Bonneville Power Administration

P.0. Box 3621

Portland, OR 97208

S. J. Hightower

Bureau of Reclamation

Denver Federal Center

Building 67, Code 254

Denver, CO 80225

R. N. Clark

Department of Agriculture

S.W. Great Plains Research Center

Bushland, TX 79012

2 E. DeMeo/F. R. Goodman

Electric Power Research Institute 3412 Hillview Avenue

Palo Alto, CA 94303

S. R. Andersen

ERT, Inc.

P.0. Box 2105

Fort Collins, CO 80522

10 H. -T. Liu

Flow Wind, Corporation

21414-68th Avenue, South

Kent, WA 98031 
No. of

Copies

J. D. West

HQ MAC/DEEE

Scott AFB, IL 62225

G. G. Worley, Maj. USAF

HQ AF-ESC/RDVA

Tynda11 AFB, FL 32403

V. F. Garrett

Montana Energy and MHD Research and Development Institute, Inc. P.0. Box 3809

Butte, MT 59701

J. Konigsberg

Montana Energy Office

Capital Station

Helena, MT 59601

5 L. Gordon

NASA/Lewis Research Center

Mai1-Stop 500-202

21000 Brookpark Road

Cleveland, $\mathrm{OH} 44135$

M. J. Changery

National Oceanic and Atmospheric Administration

National Climatic Center

Federal Building

Asheville, NC 28801

2 R. W. Baker

Atmospheric Sciences Department Oregon State University

Corvallis, OR 97331

T. J. Healy/C. Hansen

Rockwel1 International

Rocky Flats Plant

P.0. Box 464

Golden, CO 80401

E. Kadlec

Sandia Laboratories

Division 5443

P.0. Box 5800

Albuquerque, NM 87115
No. of

Copies

R. E. Akins

Sandia Laboratories

Division 5443

P. 0. Box 5800

Albuquerque, NM 87115

R. M. Traci

Science Applications, Inc.

P.0. Box 2351

La Jolla, CA 92038

R. Noun

Solar Energy Research Institute 1617 Cole Boulevard

Golden, CO 80401

2 C. M. Bhumralkar/W. Johnson

Stanford Research Institute, International

333 Ravenswood Avenue

Menlo Park, CA 94025

R. M. Endlich/J. D. Lee

Stanford Research Institute, International

333 Ravenswood Avenue

Menlo Park, CA 94025

B. Bailey

Atmospheric Sciences Research Center

State University of New York at Albany

Albany, NY 12222

N. E. Suhs

Tennessee Valley Authority

1360 Commerce Union Bank Building

Chattanooga, TN 37401

W. R. Thorn, Manager

TERA Corporation

Wind Systems Engineering

2150 Shattuck Avenue

Berkeley, CA 94704 
No. of

Copies

J.-T. Lin, President

United Industries, Corporation

12835 Bell-Red Road

Bellevue, WA 98005

A. Daniels

University of Hawaij at Manoa

Department of Meteorology

2525 Correa Road

Honolulu, HI 96822

M. Garstang

Department of Environmental Sciences

University of Virginia

Charlottesville, VA 22903

S. Hosch

Washington State Energy Office

$400 \mathrm{E}$. Union Avenue, 1st Floor

Olympia, WA 98504

F. S. Seiler

Wind Energy Report

Box 14 - 104 S. Village Avenue

Rockville Centre, NY 11571

E. L. Davis

U.S. Windpower, Inc.

6421 B. South Front Road

Livermore, CA 94550

R. L. Berry

Atmospheric Environment

4905 Dufferin Street

Downsview, Ontario

M3H 5T4

CANADA

R. J. Templin

National Research Council of Canada

National Aeronautical

Establishment

M-2 Montreal Road

Ottawa, Ontario K1A OR6

CANADA
No. of

Copies

D. Lindley

Taylor Woodrow Construction, Limited

Taywood House

345 Ruislip Road

Southha11, Middlesex UBI 2QX

ENGLAND

D. T. Swift-Hook

Head of Applied Physics Branch

Central Electricity Research Laboratories

Kelvin Avenue

Leatherhead

Surrey, KT 22

UNITED KINGDOM

P. W. Blakely

New Zealand Electricity

Rutherford House

Lambton Quay

Wellington

NEW ZEALAND

N. Cherry

Lincoln College

Canterbury

NEW ZEALAND

0 . Ljungstrom

FFA, The Aeronautical Research Institute

Forskningsstationen i Stockholm Drottning Kristinas Vag 47

S-114 29 Stockholm

SWEDEN

\section{ONSITE}

DOE Richland Operations Office

H. E. Ransom/D. R. Segna 
No. of

Copies

43 Pacific Northwest Laboratory

W. R. Barchet

J. W. Buck

J. R. Conne11

C. E. Elderkin

D. L. Elliott

R. L. George

D. L. Hadley (5)

S. K. Heflick

A. H. Miller
No. of

Copies

E. L. Owczarski

D. C. Powel1

J. J. Praino

D. S. Renné

W. F. Sandusky

H. L. Wegley

L. L. Wende 11

R. K. Woodruff

Technical Information Library (5)

Publishing Coordination (2)

WCPE Project Office (15) 